hep-ph/0602178

Imperial/TP/

ITFA-2006-07

$\mathrm{UCLA} / 06 / \mathrm{TEP} / 06$

ZU-TH $07 / 06$

\title{
The Cut-Constructible Part of QCD Amplitudes
}

\author{
Ruth Britto $^{1}$, Bo Feng ${ }^{2,3}$, Pierpaolo Mastrolia ${ }^{4,5}$ \\ ${ }^{1}$ Institute for Theoretical Physics, University of Amsterdam \\ Valckenierstraat 65, 1018 XE Amsterdam, The Netherlands \\ ${ }^{2}$ Blackett Laboratory, Imperial College, London, SW7 2AZ, UK \\ ${ }^{3}$ The Institute for Mathematical Sciences, Imperial College London \\ 48 Princes Gardens, London SW' 2AZ, UK \\ 4 Department of Physics and Astronomy, UCLA \\ Los Angeles, CA 90090-1547, USA \\ 5 Institut für Theoretische Physik, Universität Zürich \\ CH-8057 Zürich, Switzerland
}

Unitarity cuts are widely used in analytic computation of loop amplitudes in gauge theories such as QCD. We expand upon the technique introduced in hep-ph/0503132 to carry out any finite unitarity cut integral. This technique naturally separates the contributions of bubble, triangle and box integrals in one-loop amplitudes and is not constrained to any particular helicity configurations. Loop momentum integration is reduced to a sequence of algebraic operations. We discuss the extraction of the residues at higher-order poles. Additionally, we offer concise algebraic formulas for expressing coefficients of threemass triangle integrals. As an application, we compute all remaining coefficients of bubble and triangle integrals for nonsupersymmetric six-gluon amplitudes.

February 2006 


\section{Introduction}

Within the experimental programme of the forthcoming Large Hadron Collider, and the exigencies of efficient ways to analyse huge amounts of data, perturbative QCD will play the role of precision physics. To dig out interesting signals we must be able to distinguish them from backgrounds, largely dominated by QCD processes. In order to have enough theoretical accuracy for comparisons against the experimental counterpart, oneloop (and even higher) many-leg amplitudes of several QCD processes are needed [1,2]. However, the calculation of next-to-leading order (NLO) amplitudes is extremely difficult; within the current status of the available analytical results, 'many legs' means five [3] as regarding QCD corrections and six [4] as for electroweak. The most recent theoretical efforts for tackling the one-loop multi-leg amplitudes have been using algebraic/semi-numerical approaches [5].

For the analysis of jet-events produced at the high energies of the LHC, it is mandatory to overcome the bottleneck of the one-loop six-gluon amplitude.

Although it is very difficult to calculate QCD amplitudes, various methods have been developed to attack this problem. One efficient approach is the unitarity cut method with the spinor-helicity formalism [6, 7,8] (a review may be found in [9]). It is shown in [3, 10, 11] that one-loop amplitudes with all external gluons and a gluon circulating around the loop can be decomposed into following three pieces (the so-called supersymmetry decomposition),

$$
\mathcal{A}^{\mathrm{QCD}}=\mathcal{A}^{\mathcal{N}=4}-4 \mathcal{A}^{\mathcal{N}=1}+\mathcal{A}^{\text {scalar }},
$$

where $\mathcal{A}^{\mathrm{QCD}}$ denotes an amplitude with only a gluon circulating in the loop, $\mathcal{A}^{\mathcal{N}=4}$ has the full $\mathcal{N}=4$ multiplet circulating in the loop, $\mathcal{A}^{\mathcal{N}=1}$ has an $\mathcal{N}=1$ chiral supermultiplet in the loop, and $\mathcal{A}^{\text {scalar }}$ has only a complex scalar in the loop. This last term is sometimes referred to as $\mathcal{A}^{\mathcal{N}=0}$.

The main advantage of this decomposition is that supersymmetric amplitudes, $\mathcal{A}^{\mathcal{N}=4}$ and $\mathcal{A}^{\mathcal{N}=1}$, are four-dimensional cut-constructible [10,11], meaning that they do not suffer any ambiguity due to the presence of rational terms; they are completely determined by their finite unitarity cuts.

However, the term $\mathcal{A}^{\text {scalar }}$ cannot be completely reconstructed from its absorptive part, and the presence of rational functions of the kinematic invariants make its calculation quite involved, though still simpler than the full $\mathcal{A}^{\mathrm{QCD}}$. In fact, it is our aim in this paper to 
complete the program introduced in [12] to give a systematic way to evaluate the cutconstructible piece of $\mathcal{A}^{\text {scalar }}$. The determination of the rational terms could perhaps be later achieved by implementing the recursive technique introduced in [13, 14, 15]. An alternative is to apply the unitarity method in $(4-2 \epsilon)$ dimensions, for there the entire amplitude is cut-constructible [16].

Our systematic method to deal with the $\mathcal{A}^{\text {scalar }}$ part is related methods and ideas from the twistor string theory initiated in [17] and further developed in [18, 19, 20, 21]. In particular, we make heavy use of the new way to write phase space integrals and perform the integration given in [18,21]. 17 The algorithm initiated in [12] and developed here reduces phase space integration to algebraic manipulations.

With the complete algorithm developed in this paper, we calculate the (heretofore missing) cut-constructible part of the NLO six-gluon amplitudes in QCD.

\subsection{The Current Status of Amplitudes}

Under the separation given by (1.1), the unitarity method established itself as an effective means of computation [10, 11, 24, 25, 26, 27,28]. This method has been applied successfully in several contexts, supersymmetric [10,11, 29, 30, 28, 12, as well as nonsupersymmetric [31,24, 32,25]. A recent innovation [27,30, making use of leading singularities, allows a simple determination of coefficients of integrals associated to box topologies, without any explicit integration. $\mathrm{\theta}$ Some related methods are the use of MHV diagrams in the cut calculations [37] and the use [21, 38, 39] of the holomorphic anomaly [20] to determine certain cut integrals [40,21].

Although supersymmetric multiplets contain more particles, the reading of the QCD amplitudes in this supersymmetric fashion introduces a degree of simplicity in terms of computation.

The simplest term is the contribution of an $\mathcal{N}=4$ super Yang-Mills multiplet. The $\mathcal{N}=4$ amplitudes can be expressed as a combination of scalar box integral functions with rational coefficients [10]. These coefficients have been evaluated in a closed form for the case with maximal helicity violation (MHV), namely helicity configurations where two

1 For a review, see [22] and its citations. See also [23].

2 With the unitarity method, tree-level amplitudes are the bricks of the cut integral. Off-shell recursion relations [33, 34 are a well-known method to construct trees. As valid alternatives, there are two new efficient techniques, exploiting the on-shellness of the amplitudes: the MHV diagrammatic rules of [18] and the on-shell recursion relations of [35, 36]. 
gluons are of negative helicity and the rest are of positive helicity [10,37, and for the case of next-to-MHV (NMHV) amplitudes [11, 38, 26, 28, 27]. The ingredients here are the Parke-Taylor tree-level MHV amplitudes [33, 34].

In the case of an $\mathcal{N}=1$ chiral multiplet, the all- $n$ one-loop MHV and one-loop sixgluon amplitudes are known [11,37, 39, 41, 42, 12].

As we have said, the most difficult part in the decomposition (1.1) is $\mathcal{A}^{\text {scalar }}$ part, which is known only in special cases. All amplitudes with at most one negative-helicity gluon were computed in [43,44,45]. The cut parts of the MHV amplitudes are known [11,10,46] for an arbitrary number of legs. Very recently an explicit form of the rational functions has been presented for the all-multiplicity MHV amplitude in which the negative-helicity gluons are adjacent [13,15]. All box coefficients of the six-gluon amplitude are given in [42]. All cut-constructible coefficients of one-loop amplitudes where the gluons are ordered in two adjacent bunches of opposite helicity (a 'split helicity' configuration), for $\mathcal{N}=0$ and $\mathcal{N}=1$, have been computed in [47].

To achieve the complete calculation of $\mathcal{A}^{\text {scalar }}$ for the six-gluon amplitude, there are two possible paths to follow. The first is to apply the unitarity method in $(4-2 \epsilon)$ dimensions instead of four [16]. Cases with four external particles, and up to six, in special helicity configurations, have been worked out along this way [31, 48, 32, 25, 49]. Recent progress in deriving the tree-amplitude ingredients has appeared in [50,51,52,53,54, 55]. Alternatively, one can split $\mathcal{A}^{\text {scalar }}$ into a cut-constructible piece and a remaining rational function, and tackle these two pieces separately. The reason is as follows. Due to a better understanding of the recursive structure of QCD amplitudes, at tree level [35,36] and at one-loop [56], and exploiting the knowledge of their collinear and soft-behaviour, it has recently been shown [13, 14, 15] that the rational term of one-loop QCD amplitudes does have, in itself, a recursive character: given the knowledge of the coefficients of the logarithmic and polylogarithmic terms of an $n$-point amplitude, the leftover rational coefficient has been reconstructed by feeding into the recursion the rational coefficients of the $(n-1)$-point amplitudes which represent the all-channel collinear limits of the $n$-point one. Thus, if we calculate the cut-constructible part, we can try to obtain the corresponding rational part by recursion relations. Combining the results, one might obtain the complete answer for $\mathcal{A}^{\text {scalar }}$. 


\subsection{The Plan of the Paper}

The plan of this paper is as follows. In Section 2, we give the general setting for our paper. We exploit the divergent behavior of the amplitude to reduce the size of the basis of known integrals. Then we analyze the structure of our phase space integration. We show that we can neatly divide contributions into rational and logarithmic parts. For the logarithmic parts, we show where the three-mass triangle and four-mass box functions show up. Furthermore, we give our general strategy to reduce integration to algebraic manipulation. Specifically, we show how to read out residues of higher-order poles, which is one of the most important steps in this procedure.

Starting from Section 3, we describe the calculation of the cut-constructible part of $\mathcal{A}^{\text {scalar }}$ of six-gluon scattering amplitudes. Section 3 is dedicated to the helicity configuration $\left(1^{-}, 2^{-}, 3^{-}, 4^{+}, 5^{+}, 6^{+}\right)$; Section 4 , to the configuration $\left(1^{-}, 2^{+}, 3^{-}, 4^{+}, 5^{-}, 6^{+}\right)$; and Section 5 , to configuration $\left(1^{-}, 2^{-}, 3^{+}, 4^{-}, 5^{+}, 6^{+}\right)$.

The results of the rational coefficients of bubble and triangle functions are expressed, in compact form, in terms of sums over spinor products.

Concluding remarks are given in Section 6.

We supply the paper with three appendices which contain the main technical details of the calculation. Appendix A gives the NMHV tree-level amplitudes which enter the cuts. Appendix B contains a detailed analysis of logarithmic contributions in cut-integration. In Appendix C, we show how to read out the coefficients of three-mass triangles, which require the computation of one-Feynman-parameter integrals, and we define some functions we will use throughout the manuscript.

We leave to future work the automatic implementation of the whole algorithm for providing the numerical counterpart of our results. That would allow numerical checks of our results against those already existing in the literature. The non-uniqueness of the expressions in the framework of the spinor formalism, due to hidden identities among spinor products, (e.g. Schouten identities and momentum conservation), can make direct analytic comparison quite difficult. Currently, spinor algebra manipulation is frequently carried out in Mathematica, while the final integration, employing the Cauchy residue theorem and, when needed, one-Feynman-parameter representation, has not been automatized. 


\section{General Setting: Preliminaries}

In this paper, we freely use the word 'amplitude' to refer to the cut-constructible part of $\mathcal{A}^{\text {scalar }}$ for the leading-color partial amplitude of gluons.

Our purpose is to expand and develop the procedure introduced in [12], so we shall not repeat all the background information here. Rather we shall mainly just point out the new features showing up in this application. We also make use of the same notation and conventions as in [12], which follows the spinor-helicity formalism [6, [7, 8] and conventions of [17]. In particular, in the following calculations we generally omit an overall factor of $-i \frac{(4 \pi)^{2-\epsilon}}{(2 \pi)^{4-2 \epsilon}}$.

By reduction techniques, the cut-constructible portion of the amplitude may be expanded in a basis of scalar integral functions known as boxes $\left(I_{4}\right)$, triangles $\left(I_{3}\right)$, and bubbles $\left(I_{2}\right)$ [57, 11].

$$
\begin{aligned}
A_{n}=\frac{r_{\Gamma}\left(\mu^{2}\right)^{\epsilon}}{(4 \pi)^{2-\epsilon}} \sum( & c_{2} I_{2}+c_{3}^{1 m} I_{3}^{1 m}+c_{3}^{2 m} I_{3}^{2 m}+c_{3}^{3 m} I_{3}^{3 m} \\
& \left.+c_{4}^{1 m} I_{4}^{1 m}+c_{4}^{2 m e} I_{4}^{2 m e}+c_{4}^{2 m}{ }^{h} I_{4}^{2 m} h+c_{4}^{3 m} I_{4}^{3 m}+c_{4}^{4 m} I_{4}^{4 m}\right) .
\end{aligned}
$$

This defines what we mean by the cut-constructible portion of the amplitude. Here $\epsilon \equiv$ $(4-D) / 2$ is the dimensional regularization parameter, $\mu$ is the renormalization scale, and $r_{\Gamma}$ is defined by

$$
r_{\Gamma}=\frac{\Gamma(1+\epsilon) \Gamma^{2}(1-\epsilon)}{\Gamma(1-2 \epsilon)} .
$$

The sum runs over all the cyclic permutations within each type of integral.

For a gluon amplitude with a complex scalar in the loop, the infrared and ultraviolet singular behavior is [58,59,60

$$
\left.A_{n}^{\text {scalar }}\right|_{\text {singular }}=\frac{r_{\Gamma}}{3 \epsilon(4 \pi)^{2-\epsilon}} A_{n}^{\text {tree }} .
$$

Because the divergence has exactly the same $\epsilon$-dependence as $A_{n}^{\mathcal{N}}=\left.1\right|_{\text {singular }}$, we may follow the same argument as in [12] to express the amplitude in terms of a smaller, modified basis of scalar integrals with no one-mass or two-mass triangle functions:

$$
\begin{aligned}
\mathcal{A}_{n}^{\text {scalar }}=\frac{r_{\Gamma}\left(\mu^{2}\right)^{\epsilon}}{(4 \pi)^{2-\epsilon} \sum\left(c_{2} I_{2}\right.} & +c_{3}^{3 m} I_{3}^{3 m}+c_{4}^{1 m} I_{4 F}^{1 m}+c_{4}^{2 m e} I_{4 F}^{2 m} e \\
& \left.+c_{4}^{2 m}{ }^{h} I_{4 F}^{2 m}{ }^{h}+c_{4}^{3 m} I_{4 F}^{3 m}+c_{4}^{4 m} I_{4}^{4 m}\right) .
\end{aligned}
$$

This basis differs from the one in (2.1) in that each integral function (except the bubble functions) has had its divergences stripped away. See Figures 1 and 2. Precise definitions appear in Appendix A of [12]. 


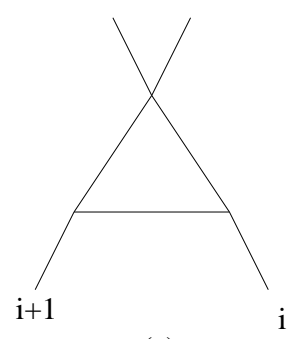

(a)

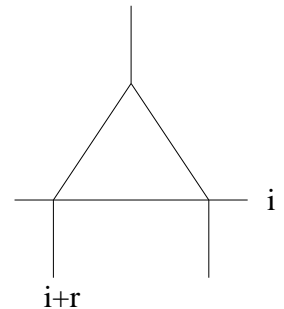

(b)

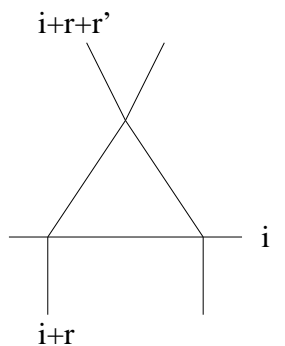

(c)

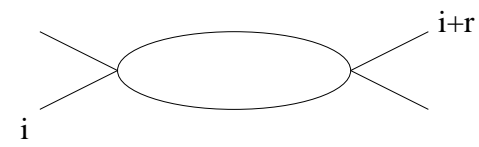

(d)

Fig. 1: Scalar bubble and triangle integrals. (a) One-mass triangle $I_{3 ; i}^{1 m}$. (b) Twomass triangle $I_{3: r ; i}^{2 m}$. (c) Three-mass triangle $I_{3: r: r^{\prime} ; i}^{3 m}$. (d) Bubble $I_{2: r ; i}$. Note that the modified basis in (2.4) involves only three-mass triangles and bubbles.

The justification for eliminating one-mass and two-mass triangles is based on the observation that according to (2.3), $\mathcal{A}_{n}^{\text {scalar }}$ diverges as $1 / \epsilon$. Since one-mass and two-mass triangle integrals are the only ones with larger, $1 / \epsilon^{2}$, divergences, these leading terms must conspire to cancel. After observing further that these $1 / \epsilon^{2}$ divergences arise only in the particular combination $(-s)^{-\epsilon} / \epsilon^{2}$, where $s$ is a momentum invariant, it follows that the contributions of one-mass and two-mass triangles may be neglected altogether as per the modified basis in (2.4).

To compute the amplitude, it is sufficient to compute each of these coefficients separately. The principle of the unitarity-based method [10,11,31] is to exploit the unitarity cuts of the scalar integrals to extract the coefficients.

\subsection{Coefficients from Unitary Cuts}

Our goal is to compute the coefficients in (2.4) by applying unitarity cuts [61] ${ }^{3}$ directly in four dimensions.

3 This technique is thoroughly discussed in [62]. While these early works were not intended to apply to massless theories, we find there is no obstacle in adapting them to our purposes. The modern interpretation is found in [10,11]. 


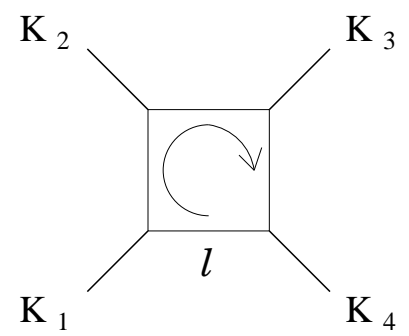

(a)

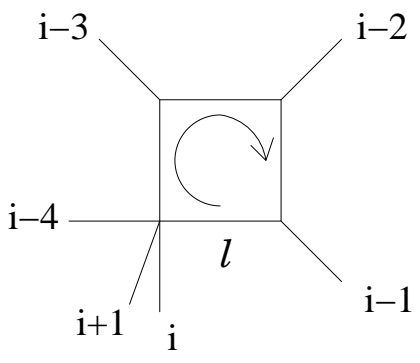

(b)

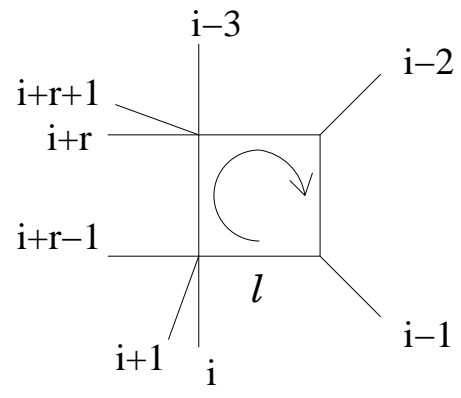

(d)

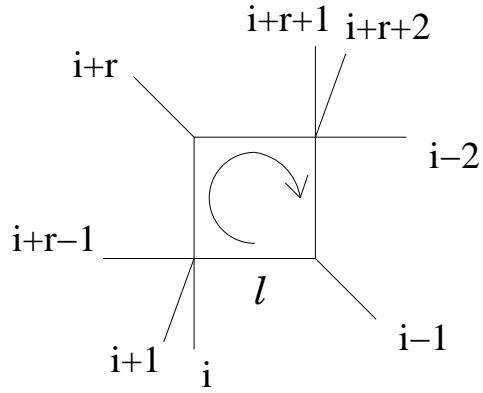

(c)

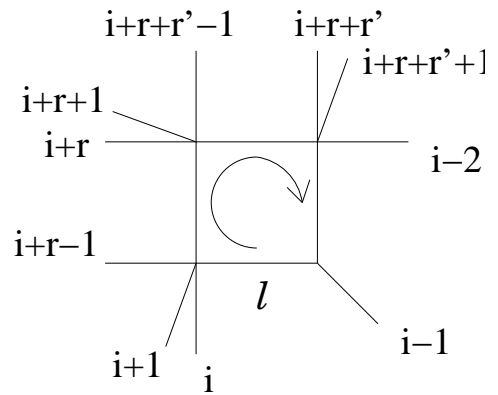

(e)

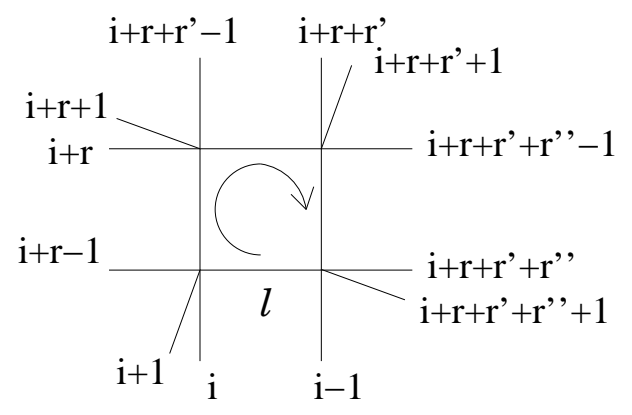

(f)

Fig. 2: Scalar box integrals. (a) The outgoing external momenta at each of the vertices are $K_{1}, K_{2}, K_{3}, K_{4}$, defined to correspond to sums of the momenta of gluons in the exact orientation shown. (b) One-mass $I_{4 ; i}^{1 m}$. (c) Two-mass "easy" $I_{4: r ; i}^{2 m e}$. (d) Two-mass "hard" $I_{4: r ; i}^{2 m}{ }^{h}$. (e) Three-mass $I_{4: r: r^{\prime} ; i}^{3 m}$. (f) Four-mass $I_{4: r: r^{\prime}: r " ; i}^{4 m}$. 


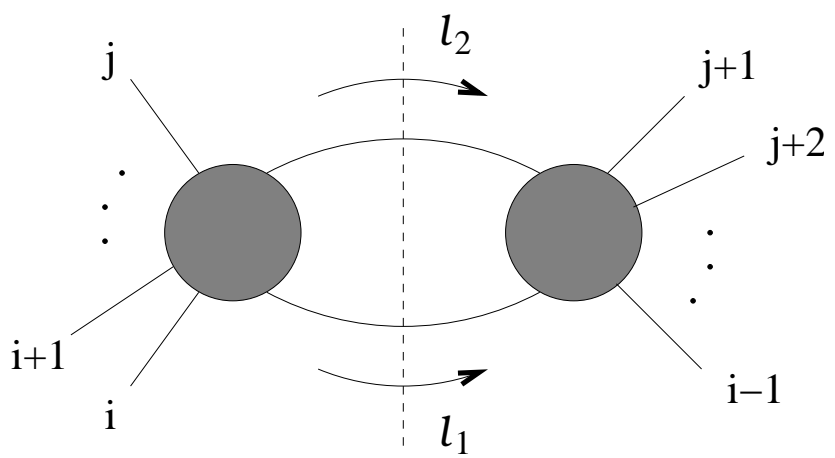

Fig. 3: Representation of the cut integral. Left and right tree-level amplitudes are on-shell. Internal lines represent the legs coming from the cut propagators.

On the right-hand side, the scalar integrals are known functions, so their discontinuities are also known explicitly. We can read out the coefficients of box integrals from quadruple cuts, while coefficients of bubbles and three-mass triangle integrals from double cuts. The procedure of [12] can be extended to box integrals as well, but since it is more efficient to use quadruple cuts, we do not explore that possibility any further here.

To be able to read out these coefficients of bubbles and three-mass triangles we need to know their discontinuities in double cuts. They are given by

$$
\Delta I_{2}\left(P_{\text {cut }}\right)=-1
$$

and

$$
\Delta I_{3}^{3 m}\left(K_{1}\right)=\int_{0}^{1} d z \frac{1}{\left(z Q+(1-z) K_{1}\right)^{2}}, \quad Q=K_{3}+\frac{K_{3}^{2}}{K_{1}^{2}} K_{1}
$$

where $K_{1}$ is the cut momentum (see [12] for detailed derivations). In fact we could carry out the integration for $\Delta I_{3}^{3 m}\left(K_{1}\right)$ as in [12]. But to read off the coefficients of three-mass triangles, we need only compare the expressions on both sides without really doing the $z$-integration, so the form (2.6) is more useful.

On the left-hand side of (2.4), the discontinuity of the amplitude in the $P_{i, j}$ momentum channel is computed by the integral

$$
\begin{aligned}
& C_{i, i+1, \ldots, j-1, j}= \\
& \int d \mu A^{\text {tree }}\left(\ell_{1}, i, i+1, \ldots, j-1, j, \ell_{2}\right) A^{\text {tree }}\left(\left(-\ell_{2}\right), j+1, j+2, \ldots, i-2, i-1,\left(-\ell_{1}\right)\right),
\end{aligned}
$$

4 The discontinuities of box functions may be found in [21]. 
where $d \mu=d^{4} \ell_{1} d^{4} \ell_{2} \delta^{(+)}\left(\ell_{1}^{2}\right) \delta^{(+)}\left(\ell_{2}^{2}\right) \delta^{(4)}\left(\ell_{1}+\ell_{2}-P_{i j}\right)$ is the Lorentz invariant phase space measure of two light-like vectors $\left(\ell_{1}, \ell_{2}\right)$ constrained by momentum conservation. See Figure 3.

We need to bring the integral (2.7) into a form convenient to work with. In a nutshell, we begin by expressing the two tree-level amplitudes in terms of spinor products. We then use the four-dimensional delta function to integrate one of the propagator momenta. Then, we use the technique of [18] to rewrite the measure in terms of spinors.

$$
\int d^{4} \ell \delta^{(+)}\left(\ell^{2}\right)(\bullet)=\int_{0}^{\infty} d t t \int\langle\lambda, d \lambda\rangle[\widetilde{\lambda}, d \widetilde{\lambda}](\bullet)
$$

where the bullets represent generic arguments, and the integration contour for the spinors is the diagonal $\mathbb{C P}^{1}$ defined by $\widetilde{\lambda}=\bar{\lambda}$.

Next, we use the remaining delta function to perform the $t$-integral. The fact that $\lambda$ and $\widetilde{\lambda}$ are independent homogeneous coordinates on two copies of $\mathbb{C P}^{1}$ means that the result must be homogeneous in $\lambda$ and $\widetilde{\lambda}$. In particular, it may be written as a sum of terms of the form

$$
\frac{1}{\left\langle\ell\left|P_{c u t}\right| \ell\right]^{n}} \frac{\prod\left[a_{i} \ell\right] \prod\left\langle b_{j} \ell\right\rangle \prod\left\langle\ell\left|Q_{k}\right| \ell\right]}{\prod\left[c_{i} \ell\right] \prod\left\langle d_{j} \ell\right\rangle \prod\left\langle\ell\left|O_{k}\right| \ell\right]}, \quad O_{k} \neq P_{\text {cut }}, \quad O_{k}^{2} \neq 0
$$

where $P_{\text {cut }}$ is the momentum in the channel of the corresponding double cut and the $O_{k}$ are massive.

There are two key features of the form (2.9). The first key feature is that the degrees of both $\lambda$ and $\widetilde{\lambda}$ are -2 , which is consistent with the scaling of the integration measure $\int\langle\ell d \ell\rangle[\ell d \ell]$.

The second key feature is that among all kinds of factors in denominators, namely $\left\langle\ell\left|P_{c u t}\right| \ell\right],[c \ell],\langle d \ell\rangle$ and $\langle\ell|O| \ell]$, only the factor $\left\langle\ell\left|P_{c u t}\right| \ell\right]$ can appear with power greater than one. The reason is clear: the factor $\left\langle\ell\left|P_{\text {cut }}\right| \ell\right]$ comes from $t$-integration, so in principle we can have an arbitrary power, 5 while the other three factors $[c \ell],\langle d \ell\rangle$ and $\langle\ell|O| \ell]$, with $O^{2} \neq 0$, come from tree-level amplitudes, which have only single poles.

5 The cases $n=1$ and $n=0$ are degenerate. For example, the $n=0$ case shows up in Appendix A of [27]. 


\subsection{Canonical Decomposition}

Now we discuss the canonical decomposition procedure given in [12]. When we do the decomposition we need to choose which variables, $\lambda$ or $\tilde{\lambda}$, to be reduced. For the general discussion in this section and in Appendix $\mathrm{B}$ we reduce $\tilde{\lambda}$ variables, but in the explicit examples, we may reduce $\lambda$, depending on the situation.

First, we should split the various single poles by partial fractioning, using the following identity:

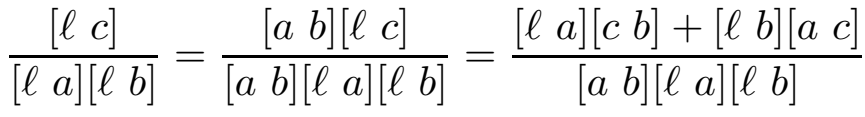

$$
\begin{aligned}
& =\frac{\left[\begin{array}{ll}
c & b
\end{array}\right]}{\left[\begin{array}{ll}
a & b
\end{array}\right]} \frac{1}{[\ell b]}+\frac{\left[\begin{array}{ll}
a & c
\end{array}\right]}{\left[\begin{array}{ll}
a & b
\end{array}\right]} \frac{1}{[\ell a]},
\end{aligned}
$$

and its generalization, where the degree of $\widetilde{\lambda}$ in both numerator and denominator decreases by one. It is worth noting that in the process of splitting, we may have the following factors in the denominator: $\left\langle\ell\left|O_{k}\right| c_{i}\right]$ or $\left\langle\ell\left|O_{k} O_{j}\right| \ell\right\rangle$. But the important point is that these factors appear only once, i.e., they are all single poles.

After splitting all single poles we end up with factors $6\left(\left\langle\ell\left|P_{c u t}\right| \ell\right]^{n}[\ell a]\right)^{-1}$ or $\left(\left\langle\ell\left|P_{\text {cut }}\right| \ell\right]^{n}\langle\ell|Q| \ell]\right)^{-1}$. Then we need to perform the same splitting of $\left\langle\ell\left|P_{\text {cut }}\right| \ell\right]$ and $[\ell a]$ (or $\left\langle\ell\left|P_{\text {cut }}\right| \ell\right]$ and $\langle\ell|Q| \ell]$ ). After finishing this step we have the following types of terms:
(1) $\frac{G(\lambda, \tilde{\lambda})}{\left\langle\ell\left|P_{\text {cut }}\right| a\right]^{m+1}\left\langle\ell\left|P_{\text {cut }}\right| \ell\right]^{n-m}}$,
(2) $\frac{G(\lambda, \widetilde{\lambda})}{\left\langle\ell\left|P_{\text {cut }} Q\right| \ell\right\rangle^{m+1}\left\langle\ell\left|P_{\text {cut }}\right| \ell\right]^{n-m}}$,
(3) $\frac{F(\lambda)}{\left\langle\ell\left|P_{\text {cut }}\right| a\right]^{n-1}} \frac{1}{\left\langle\ell\left|P_{\text {cut }}\right| \ell\right][\ell a]}$,
(4) $\frac{F(\lambda)}{\left\langle\ell\left|P_{\text {cut }} Q\right| \ell\right\rangle^{n-1}} \frac{1}{\left\langle\ell\left|P_{\text {cut }}\right| \ell\right]\langle\ell|Q| \ell]}$,

where $G(\lambda, \tilde{\lambda})$ is a function of both $\lambda$ and $\tilde{\lambda}$ while $F(\lambda)$ is a function of $\lambda$ only. One important thing for both functions $G(\lambda, \widetilde{\lambda})$ and $F(\lambda)$ is that they have only single poles.

The results in (2.11) are our final results for the canonical decomposition. There are several points to be explained. First, we have multiple poles like $\left\langle\ell\left|P_{\text {cut }}\right| a\right]$ or $\left\langle\ell\left|P_{\text {cut }} Q\right| \ell\right\rangle$, so we need to discuss how to read out residues of these multiple poles. Second, as we will analyze carefully in Appendix B, terms of types (1) and (2) will contribute to rational functions while terms of types (3) and (4) will contribute to pure logarithmic functions. Thirdly, type (3) will only contribute to one-mass, two-mass and three-mass box functions, while type (4) will contribute in addition to three-mass triangle and four-mass box functions. The reason is because $Q^{2} \neq 0$ in type (4). Since box coefficients are easily obtained from quadruple cuts, we will pay the most attention to type (4).

\footnotetext{
6 As we have remarked, for degenerate cases we may end up with factors like $(\langle\ell|O| \ell]\langle\ell|Q| \ell])^{-1}$ or $([\ell a][\ell b])^{-1}$, but the discussion applies to these cases as well.
} 


\subsection{Rewriting as Total Derivative}

Before proceeding to extract residues of multiple poles, let us recall the strategy of integration. The key idea is to write $\int\langle\ell d \ell\rangle[\ell d \ell] G(\lambda, \widetilde{\lambda})$ in the form $\int\langle\ell d \ell\rangle\left[d \ell \partial_{\ell}\right] \widetilde{G}(\lambda, \widetilde{\lambda})$. Then the integration is reduced to algebraic manipulation by reading off residues at poles in $\widetilde{G}(\lambda, \widetilde{\lambda})$. One useful formula is given by 12

$$
\begin{aligned}
& \frac{[\ell d \ell] \prod_{i=1}^{j+1}\left[\eta_{i} \ell\right]\left[\eta_{j+2} \ell\right]^{n-j-1}}{\langle\ell|P| \ell]^{n+2}}=\left[d \ell \partial_{\ell}\right]\left[\frac{\prod_{i=1}^{j+1}\left\langle\ell|P| \eta_{i}\right]}{\langle\ell|P| \ell]^{n+1}}\right. \\
& \left.\times\left(\sum_{k=0}^{j+1} \frac{(-1)^{j+1-k}(j+1-k) !}{(n+1-(j+1))(n+1-j) \cdots(n+1-k)} g_{k}\left[x_{s}\right] \frac{\left[\eta_{j+2} \ell\right]^{n+1-k}}{\left\langle\ell|P| \eta_{j+2}\right]^{(j+1)+1-k}}\right)\right],
\end{aligned}
$$

where $\eta$ is an arbitrary but fixed auxiliary spinor and

$$
g_{k}\left[x_{i}\right]=\sum_{i_{1}<i_{2}<\cdots<i_{k}} x_{i_{1}} x_{i_{2}} \cdots x_{i_{k}}, \quad \text { with } x_{i}=\frac{\left[\eta_{i} \ell\right]}{\left\langle\ell|P| \eta_{i}\right]} .
$$

One special case is when all the $\eta_{i}$ are the same and we choose the auxiliary spinor to be same $\eta$ as well. Then we have

$$
[\ell d \ell]\left(\frac{[\eta \ell]^{n}}{\langle\ell|P| \ell]^{n+2}}\right)=\left[d \ell \partial_{\ell}\right]\left(\frac{1}{(n+1)} \frac{1}{\langle\ell|P| \eta]} \frac{[\eta \ell]^{n+1}}{\langle\ell|P| \ell]^{n+1}}\right),
$$

where no multiple poles show up. We will use (2.14) often in later calculations.

For terms of types (1) and (2) we can use the formula (2.12) directly, so the results are just pure rational functions from residues of poles. But for type (3) and (4) we need to use Feynman parametrization first to write it in our standard form, as in (2.12), and then read out residues. After that we need to integrate the Feynman parameter. Notice that the order of reading out residues versus Feynman parameter integration is important. In Appendix B we discuss with care the properties of this integration.

\subsection{The Residues of Multiple Poles}

As we have seen, in general there are multiple poles in $\widetilde{G}(\lambda, \widetilde{\lambda})$ after we rewrite the integral in the form $\int\langle\ell d \ell\rangle\left[d \ell \partial_{\ell}\right] \widetilde{G}(\lambda, \widetilde{\lambda})$. We need to know how to read off residues at these multiple poles. 0

7 In [12], no multiple poles were encountered. We do not know whether it is a general feature of supersymmetric theories that no multiple poles show up. 
The main idea is the following. Recall the underlying complex analysis. To obtain the residue of $\oint d z z^{-n} f(z)$, we need to take the $(n-1)$-th derivative of the function $f(z)$ and set $z=0$. One important consequence of the above result is that if the degree of polynomial function of $f(z)$ is less than $(n-1)$, we get zero contribution.

For our problem, notice that the degree of $\lambda$ is -2 in $\widetilde{G}(\lambda, \widetilde{\lambda})$, so if we split $\widetilde{G}(\lambda, \widetilde{\lambda})$ using the identity

$$
\frac{\langle\ell a\rangle}{\langle\ell \eta\rangle\langle\ell b\rangle}=\frac{\langle\eta a\rangle}{\langle\eta b\rangle} \frac{1}{\langle\ell \eta\rangle}+\frac{\langle b a\rangle}{\langle b \eta\rangle} \frac{1}{\langle\ell b\rangle},
$$

then at the end of splitting process we will have terms like $\frac{1}{\langle\ell \eta\rangle^{2}}, \frac{\langle\ell a\rangle}{\langle\ell \eta\rangle^{3}}, \frac{\langle\ell a\rangle\langle\ell b\rangle}{\langle\ell \eta\rangle^{4}}$ etc. (or in general, taking the form $\frac{P_{n-2}(\lambda)}{\langle\ell \eta\rangle^{n}}$ ) for the multiple poles. However, since the degree of $\lambda$ in numerator is two less than the degree of $\lambda$ in denominator, by similar reasoning the residues of all these pieces will be zero.

Now we demonstrate our strategy in several examples.

\section{The Double Pole Contribution}

Let us start from a term with a double pole,

$$
I_{2}=\frac{1}{\langle\ell \eta\rangle^{2}} \frac{\prod_{j=1}^{N}\left\langle\ell a_{j}\right\rangle}{\prod_{k=1}^{N}\left\langle\ell b_{k}\right\rangle} .
$$

Using (2.15) once, we get

$$
I_{2}=\frac{\left\langle\eta a_{1}\right\rangle}{\left\langle\eta b_{1}\right\rangle} \frac{1}{\langle\ell \eta\rangle^{2}} \frac{\prod_{j=2}^{N}\left\langle\ell a_{j}\right\rangle}{\prod_{k=2}^{N}\left\langle\ell b_{k}\right\rangle}+\frac{1}{\langle\ell \eta\rangle} \frac{\left\langle a_{1} b_{1}\right\rangle}{\left\langle\eta b_{1}\right\rangle\left\langle\ell a_{1}\right\rangle} \frac{\prod_{j=1}^{N}\left\langle\ell a_{j}\right\rangle}{\prod_{k=1}^{N}\left\langle\ell b_{k}\right\rangle} .
$$

The second term already has just a single pole, namely $\langle\ell \eta\rangle$, while the first term still has a double pole. We use (2.15) for the first term again and get

$$
\begin{aligned}
I_{2} & =\frac{1}{\langle\ell \eta\rangle} \frac{\left\langle a_{1} b_{1}\right\rangle}{\left\langle\eta b_{1}\right\rangle\left\langle\ell a_{1}\right\rangle} \frac{\prod_{j=1}^{N}\left\langle\ell a_{j}\right\rangle}{\prod_{k=1}^{N}\left\langle\ell b_{k}\right\rangle}+\frac{\left\langle\eta a_{1}\right\rangle}{\left\langle\eta b_{1}\right\rangle} \frac{1}{\langle\ell \eta\rangle} \frac{\left\langle a_{2} b_{2}\right\rangle}{\left\langle\eta b_{2}\right\rangle\left\langle\ell a_{2}\right\rangle} \frac{\prod_{j=2}^{N}\left\langle\ell a_{j}\right\rangle}{\prod_{k=2}^{N}\left\langle\ell b_{k}\right\rangle} \\
& +\frac{\left\langle\eta a_{1}\right\rangle}{\left\langle\eta b_{1}\right\rangle} \frac{\left\langle\eta a_{2}\right\rangle}{\left\langle\eta b_{2}\right\rangle} \frac{1}{\langle\ell \eta\rangle^{2}} \frac{\prod_{j=3}^{N}\left\langle\ell a_{j}\right\rangle}{\prod_{k=3}^{N}\left\langle\ell b_{k}\right\rangle} .
\end{aligned}
$$

Iterating this step, we eventually reach the final result:

$$
I_{2}=\frac{1}{\langle\ell \eta\rangle} \sum_{m=0}^{N-1} \frac{\prod_{i}^{m}\left\langle\eta a_{i}\right\rangle}{\prod_{i}^{m}\left\langle\eta b_{i}\right\rangle} \frac{\left\langle a_{m+1} b_{m+1}\right\rangle}{\left\langle\eta b_{m+1}\right\rangle\left\langle\ell a_{m+1}\right\rangle} \frac{\prod_{j=m+1}^{N}\left\langle\ell a_{j}\right\rangle}{\prod_{k=m+1}^{N}\left\langle\ell b_{k}\right\rangle}+\frac{1}{\langle\ell \eta\rangle^{2}} \frac{\prod_{i}^{N}\left\langle\eta a_{i}\right\rangle}{\prod_{i}^{N}\left\langle\eta b_{i}\right\rangle} .
$$


The second term does not contribute, as we have argued, while the first term gives the following residue at the pole $|\ell\rangle=|\eta\rangle$ :

$$
P_{2}\left[|\eta\rangle, L_{a}, L_{b}\right]=\frac{\prod_{i}^{N}\left\langle\eta a_{i}\right\rangle}{\prod_{i}^{N}\left\langle\eta b_{i}\right\rangle} \sum_{i=1}^{N} \frac{\left\langle a_{i} b_{i}\right\rangle}{\left\langle\eta b_{i}\right\rangle\left\langle\eta a_{i}\right\rangle},
$$

where the subscript "2" indicates a double pole at the spinor $|\eta\rangle$. For ease of presentation we have also defined two lists, $L_{a}=\left\{a_{1}, a_{2}, \ldots, a_{N}\right\}$ and $L_{b}=\left\{b_{1}, b_{2}, \ldots, b_{N}\right\}$.

Let us do one brief example to illustrate the result (2.19). Consider the integral

$$
\int d \mu \frac{\langle\ell a\rangle[b \ell]}{\langle\ell|P| \ell]^{3}}
$$

Using (2.12) we can write it as

$$
\int\langle\ell d \ell\rangle\left[\tilde{\ell} \widetilde{\ell} \partial_{\tilde{\ell}^{-}}\left(\frac{\langle\ell a\rangle[b \ell][\eta \ell]}{\langle\ell|P| \ell]^{2}\langle\ell|P| \eta]}-\frac{\langle\ell a\rangle\langle\ell|P| b][\eta \ell]^{2}}{2\langle\ell|P| \ell]^{2}\langle\ell|P| \eta]^{2}}\right) .\right.
$$

Now we can do the integral in two ways. The first is to choose $\mid \eta]=\mid b]$ to eliminate the double pole. The result is given by $-\frac{\langle a|P| b]}{2\left(P^{2}\right)^{2}}$.

For the second way we just let $\eta$ remain arbitrary. The first term gives

$$
-\frac{\langle a|P| \eta]\langle\eta|P| b]}{\left(P^{2}\right)^{2}\langle\eta|P| \eta]}=-\frac{\langle a|P| b]}{\left(P^{2}\right)^{2}}-\frac{\langle a \eta\rangle[\eta b]}{\left(P^{2}\right)\langle\eta|P| \eta]}
$$

The second term has a double pole at $|\ell\rangle=|P| \eta]$. Then we use the formula (2.19) to get the result. First, we have $[\eta \ell]^{2}=\langle\eta|P| \eta]^{2}$. Second, we have $\left.\left|a_{1}\right\rangle=|a\rangle,\left|a_{2}\right\rangle=|P| b\right]$, $\left.\left|b_{1}\right\rangle=\left|b_{2}\right\rangle=|P| \ell\right]=\left(-P^{2}\right)|\eta\rangle$ where we have used the fact that at the pole $\left.\left.\mid \ell\right] \rightarrow|P| \eta\right\rangle$. Putting it together, we have

$$
\begin{aligned}
& \frac{\langle\eta|P| \eta]^{2}}{2} \frac{\langle a|P| \eta][\eta b]}{\left(P^{2}\right)\langle\eta|P| \eta]^{2}}\left(\frac{\langle a \eta\rangle}{\langle\eta|P| \eta]\langle a|P| \eta]}+\frac{\langle\eta|P| b]}{\langle\eta|P| \eta] P^{2}[\eta b]}\right) \\
& =\frac{\langle a|P| \eta][\eta b]}{2\left(P^{2}\right)} \frac{\left(2 P^{2}\langle a \eta\rangle[\eta b]+\langle a|P| b]\langle\eta|P| \eta]\right)}{\langle\eta|P| \eta]\langle a|P| \eta] P^{2}[\eta b]} \\
& =\frac{\left(2 P^{2}\langle a \eta\rangle[\eta b]+\langle a|P| b]\langle\eta|P| \eta]\right)}{2 P^{2}\langle\eta|P| \eta] P^{2}} \\
& =\frac{\langle a \eta\rangle[\eta b]}{\left(P^{2}\right)\langle\eta|P| \eta]}+\frac{\langle a|P| b]}{2\left(P^{2}\right)^{2}} \\
& =-\frac{\langle a|P| b]}{2\left(P^{2}\right)^{2}},
\end{aligned}
$$


in agreement with the first method.

\section{The Triple Pole Contribution}

Now we consider the triple pole given by

$$
I_{3}=\frac{\langle\ell \xi\rangle}{\langle\ell \eta\rangle^{3}} \frac{\prod_{j=1}^{N}\left\langle\ell a_{j}\right\rangle}{\prod_{k=1}^{N}\left\langle\ell b_{k}\right\rangle} .
$$

Using (2.15) once, we get

$$
I_{3}=\frac{\left\langle\eta a_{1}\right\rangle}{\left\langle\eta b_{1}\right\rangle} \frac{\langle\ell \xi\rangle}{\langle\ell \eta\rangle^{3}} \frac{\prod_{j=2}^{N}\left\langle\ell a_{j}\right\rangle}{\prod_{k=2}^{N}\left\langle\ell b_{k}\right\rangle}+\frac{1}{\langle\ell \eta\rangle^{2}} \frac{\left\langle a_{1} b_{1}\right\rangle\langle\ell \xi\rangle}{\left\langle\eta b_{1}\right\rangle\left\langle\ell a_{1}\right\rangle} \frac{\prod_{j=1}^{N}\left\langle\ell a_{j}\right\rangle}{\prod_{k=1}^{N}\left\langle\ell b_{k}\right\rangle} .
$$

The second term has a double pole, which can be processed using (2.19). Using (2.15) again on the first term we get

$$
\begin{aligned}
I_{3}= & \frac{1}{\langle\ell \eta\rangle^{2}} \frac{\left\langle a_{1} b_{1}\right\rangle\langle\ell \xi\rangle}{\left\langle\eta b_{1}\right\rangle\left\langle\ell a_{1}\right\rangle} \frac{\prod_{j=1}^{N}\left\langle\ell a_{j}\right\rangle}{\prod_{k=1}^{N}\left\langle\ell b_{k}\right\rangle}+\frac{1}{\langle\ell \eta\rangle^{2}} \frac{\left\langle\eta a_{1}\right\rangle}{\left\langle\eta b_{1}\right\rangle} \frac{\left\langle a_{2} b_{2}\right\rangle\langle\ell \xi\rangle}{\left\langle\eta b_{2}\right\rangle\left\langle\ell a_{2}\right\rangle} \frac{\prod_{j=2}^{N}\left\langle\ell a_{j}\right\rangle}{\prod_{k=2}^{N}\left\langle\ell b_{k}\right\rangle} \\
& +\frac{\left\langle\eta a_{1}\right\rangle}{\left\langle\eta b_{1}\right\rangle} \frac{\left\langle\eta a_{2}\right\rangle}{\left\langle\eta b_{2}\right\rangle} \frac{\langle\ell \xi\rangle}{\langle\ell \eta\rangle^{3}} \frac{\prod_{j=3}^{N}\left\langle\ell a_{j}\right\rangle}{\prod_{k=3}^{N}\left\langle\ell b_{k}\right\rangle} .
\end{aligned}
$$

Continuing in this way we get

$$
I_{3}=\frac{1}{\langle\ell \eta\rangle^{2}} \sum_{m=0}^{N-1} \frac{\prod_{i}^{m}\left\langle\eta a_{i}\right\rangle}{\prod_{i}^{m}\left\langle\eta b_{i}\right\rangle} \frac{\left\langle a_{m+1} b_{m+1}\right\rangle\langle\ell \xi\rangle}{\left\langle\eta b_{m+1}\right\rangle\left\langle\ell a_{m+1}\right\rangle} \frac{\prod_{j=m+1}^{N}\left\langle\ell a_{j}\right\rangle}{\prod_{k=m+1}^{N}\left\langle\ell b_{k}\right\rangle}+\frac{\langle\ell \xi\rangle}{\langle\ell \eta\rangle^{3}} \frac{\prod_{i}^{N}\left\langle\eta a_{i}\right\rangle}{\prod_{i}^{N}\left\langle\eta b_{i}\right\rangle} .
$$

Now we can use the result (2.19) to read out the contribution at the triple pole. It is given by

$$
\begin{aligned}
& P_{3}\left[|\eta\rangle, L_{a}, L_{b}\right]= \\
& \frac{\langle\eta \xi\rangle \prod_{i=1}^{N}\left\langle\eta a_{i}\right\rangle}{\prod_{i=1}^{N}\left\langle\eta b_{i}\right\rangle}\left(\sum_{1 \leq i \leq j \leq N} \frac{\left\langle a_{i} b_{i}\right\rangle}{\left\langle\eta b_{i}\right\rangle\left\langle\eta a_{i}\right\rangle} \frac{\left\langle a_{j} b_{j}\right\rangle}{\left\langle\eta b_{j}\right\rangle\left\langle\eta a_{j}\right\rangle}+\sum_{i=1}^{N} \frac{\left\langle a_{i} b_{i}\right\rangle}{\left\langle\eta b_{i}\right\rangle\left\langle\eta a_{i}\right\rangle} \frac{\left\langle\xi a_{i}\right\rangle}{\langle\eta \xi\rangle\left\langle\eta a_{i}\right\rangle}\right)
\end{aligned}
$$

where the two lists given as arguments are $L_{a}=\left\{a_{1}, a_{2}, \ldots, a_{N}, \xi\right\}$ and $L_{b}=\left\{b_{1}, b_{2}, \ldots, b_{N}\right\}$.

\section{Higher Multiplicity Poles}

We will not give the residue of a general multiple pole explicitly, but one can now see how the procedure continues step by step. Defining

$$
I_{n}=\frac{\prod_{j=1}^{n-2}\left\langle\ell \xi_{j}\right\rangle}{\langle\ell \eta\rangle^{n}} \frac{\prod_{k=1}^{N}\left\langle\ell a_{k}\right\rangle}{\prod_{k=1}^{N}\left\langle\ell b_{k}\right\rangle}
$$


we have the following relation:

$I_{n}=\frac{\prod_{j=2}^{n-2}\left\langle\ell \xi_{j}\right\rangle}{\langle\ell \eta\rangle^{n-1}} \sum_{m=0}^{N-1} \frac{\prod_{i}^{m}\left\langle\eta a_{i}\right\rangle}{\prod_{i}^{m}\left\langle\eta b_{i}\right\rangle} \frac{\left\langle a_{m+1} b_{m+1}\right\rangle\left\langle\ell \xi_{1}\right\rangle}{\left\langle\eta b_{m+1}\right\rangle\left\langle\ell a_{m+1}\right\rangle} \frac{\prod_{j=m+1}^{N}\left\langle\ell a_{j}\right\rangle}{\prod_{k=m+1}^{N}\left\langle\ell b_{k}\right\rangle}+\frac{\prod_{j=1}^{n-2}\left\langle\ell \xi_{j}\right\rangle}{\langle\ell \eta\rangle^{n}} \frac{\prod_{i}^{N}\left\langle\eta a_{i}\right\rangle}{\prod_{i}^{N}\left\langle\eta b_{i}\right\rangle}$,

where the last term does not contribute to the residue, while the first term will give the residue of poles of one lower multiplicity according to the formula for $P_{n-1}\left[|\eta\rangle, L_{a}, L_{b}\right]$.

3. $A\left(1^{-}, 2^{-}, 3^{-}, 4^{+}, 5^{+}, 6^{+}\right)$

In this section we demonstrate the principles outlined above, in the case of $A\left(1^{-}, 2^{-}, 3^{-}, 4^{+}, 5^{+}, 6^{+}\right)$, the simplest of the three NMHV helicity configurations of six gluons. The contribution to the cut constructible part of $\mathcal{A}^{\text {scalar }}$ in this 'split helicity' configuration has already appeared in the literature [47] and was derived there by a recursive technique.

For this configuration of external gluons, there are no contributions from box or triangle integrals, as explained in [12]. The amplitude may be expressed in terms of bubble integrals alone. So the cut integrals will turn out to be rational functions.

The nonvanishing cuts are $C_{34}, C_{61}, C_{234}$ and $C_{345}$. This amplitude is invariant under a $\mathbb{Z}_{2}$ symmetry generated by $\beta: 1 \leftrightarrow 3,4 \leftrightarrow 6$. Under this symmetry, the cuts are related through the relations $\beta\left(C_{234}\right)=C_{612}$ and $\beta\left(C_{61}\right)=C_{34}$. Thus there are only two independent integrals, say $C_{34}$ and $C_{612}$.

These two cuts are given as follows. For the cut $C_{34}$ we have

$$
\begin{aligned}
& C_{34}=\int d \mu A^{\text {tree }}\left(\ell_{1}^{ \pm}, 5^{+}, 6^{+}, 1^{-}, 2^{-}, \ell_{2}^{\mp}\right) A^{\text {tree }}\left(\left(-\ell_{2}\right)^{ \pm}, 3^{-}, 4^{+},\left(-\ell_{1}\right)^{\mp}\right) \\
& =\int d \mu\left[\frac{\left\langle\ell_{2}|1+2| 6\right]^{3}}{\left[\begin{array}{ll}
6 & 1
\end{array}\right]\left[\begin{array}{ll}
1 & 2
\end{array}\right]\left\langle\ell_{2} \ell_{1}\right\rangle\left\langle\ell_{1} 5\right\rangle P_{612}^{2}\langle 5|6+1| 2]}\left(-\frac{\left\langle\ell_{1}|1+2| 6\right]}{\left\langle\ell_{2}|1+2| 6\right]}\right)^{2} \frac{\left\langle 3 \ell_{1}\right\rangle^{2}\left\langle 3 \ell_{2}\right\rangle^{2}}{\left\langle\ell_{2} 3\right\rangle\langle 34\rangle\left\langle 4 \ell_{1}\right\rangle\left\langle\ell_{1} \ell_{2}\right\rangle}\right. \\
& \left.+\frac{\left\langle 1|5+6| \ell_{1}\right]^{3}}{\left[2 \ell_{2}\right]\left[\ell_{2} \ell_{1}\right]\langle 56\rangle\langle 61\rangle P_{561}^{2}\langle 5|6+1| 2]}\left(\frac{\left\langle 1|5+6| \ell_{2}\right]}{\left\langle 1|5+6| \ell_{1}\right]}\right)^{2} \frac{\left[4 \ell_{1}\right]^{2}\left[4 \ell_{2}\right]^{2}}{\left[\ell_{2} 3\right][34]\left[4 \ell_{1}\right]\left[\ell_{1} \ell_{2}\right]}\right] \\
& +\left[\frac{\left\langle\ell_{1}|1+2| 6\right]^{4}}{\left[\begin{array}{ll}
6 & 1
\end{array}\right]\left[\begin{array}{ll}
1 & 2
\end{array}\right]\left\langle\ell_{2} \ell_{1}\right\rangle\left\langle\ell_{1} 5\right\rangle P_{612}^{2}\langle 5|6+1| 2]\left\langle\ell_{2}|1+2| 6\right]}\left(\frac{\left\langle\ell_{2}|1+2| 6\right]}{\left\langle\ell_{1}|1+2| 6\right]}\right)^{2} \frac{\left\langle 3 \ell_{1}\right\rangle^{2}\left\langle 3 \ell_{2}\right\rangle^{2}}{\left\langle\ell_{2} 3\right\rangle\langle 34\rangle\left\langle 4 \ell_{1}\right\rangle\left\langle\ell_{1} \ell_{2}\right\rangle}\right. \\
& \left.+\frac{\left\langle 1|5+6| \ell_{2}\right]^{4}}{\left[2 \ell_{2}\right]\left[\ell_{2} \ell_{1}\right]\langle 56\rangle\langle 61\rangle P_{561}^{2}\langle 5|6+1| 2]\left\langle 1|5+6| \ell_{1}\right]}\left(-\frac{\left\langle 1|5+6| \ell_{1}\right]}{\left\langle 1|5+6| \ell_{2}\right]}\right)^{2} \frac{\left[4 \ell_{1}\right]^{2}\left[4 \ell_{2}\right]^{2}}{\left[\ell_{2} 3\right][3 \quad 4]\left[4 \ell_{1}\right]\left[\ell_{1} \ell_{2}\right]}\right] \text {, }
\end{aligned}
$$


where the first square bracket uses the upper choice of helicities of the cut propagators and the second, the lower. The expression may be simplified to get

$$
\begin{aligned}
& C_{34}=2 \int d \mu\left[-\frac{\left\langle\ell_{2}|1+2| 6\right]\left\langle\ell_{1}|1+2| 6\right]^{2}}{\left[\begin{array}{lll}
6 & 1][1 & 2
\end{array}\right]\left\langle\ell_{2} \ell_{1}\right\rangle\left\langle\ell_{1} 5\right\rangle P_{612}^{2}\langle 5|6+1| 2]} \frac{\left\langle 3 \ell_{1}\right\rangle^{2}\left\langle 3 \ell_{2}\right\rangle}{\langle 34\rangle\left\langle 4 \ell_{1}\right\rangle\left\langle\ell_{1} \ell_{2}\right\rangle}\right. \\
& \left.+\frac{\left\langle 1|5+6| \ell_{1}\right]\left\langle 1|5+6| \ell_{2}\right]^{2}}{\left[2 \ell_{2}\right]\left[\ell_{2} \ell_{1}\right]\langle 56\rangle\langle 61\rangle P_{561}^{2}\langle 5|6+1| 2]} \frac{\left[4 \ell_{1}\right]\left[4 \ell_{2}\right]^{2}}{\left[\ell_{2} 3\right][3 \quad 4]\left[\ell_{1} \ell_{2}\right]}\right] \text {. }
\end{aligned}
$$

For the cut $C_{612}$ we have

$$
\begin{aligned}
& C_{612}=\int d \mu A^{\text {tree }}\left(\ell_{1}^{ \pm}, 6^{+}, 1^{-}, 2^{-}, \ell_{2}^{\mp}\right) A^{\text {tree }}\left(\left(-\ell_{2}\right)^{ \pm}, 3^{-}, 4^{+}, 5^{+},\left(-\ell_{1}\right)^{\mp}\right)
\end{aligned}
$$

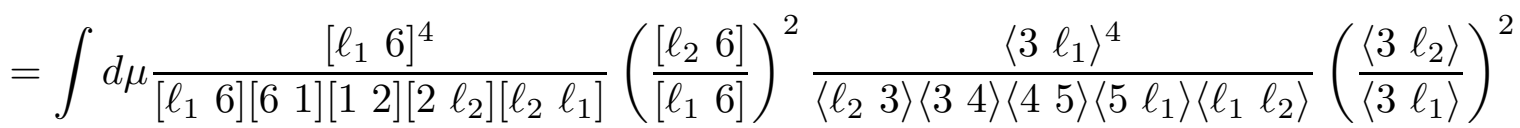

$$
\begin{aligned}
& +\frac{\left[\begin{array}{ll}
6 & \ell_{2}
\end{array}\right]^{4}}{\left[\begin{array}{ll}
\ell_{1} & 6
\end{array}\right]\left[\begin{array}{ll}
6 & 1
\end{array}\right]\left[\begin{array}{ll}
1 & 2
\end{array}\right]\left[\begin{array}{ll}
2 & \ell_{2}
\end{array}\right]\left[\begin{array}{ll}
\ell_{2} & \ell_{1}
\end{array}\right]}\left(\frac{\left[\ell_{1} 6\right.}{\left[\begin{array}{ll}
\ell_{2} & 6
\end{array}\right]}\right)^{2} \frac{\left\langle 3 \ell_{2}\right\rangle^{4}}{\left\langle\ell_{2} 3\right\rangle\langle 34\rangle\langle 45\rangle\left\langle 5 \ell_{1}\right\rangle\left\langle\ell_{1} \ell_{2}\right\rangle}\left(\frac{\left\langle 3 \ell_{1}\right\rangle}{\left\langle 3 \ell_{2}\right\rangle}\right)^{2} \\
& \left.=2 \int d \mu \frac{\left[\begin{array}{ll}
6 & \ell_{2}
\end{array}\right]^{2}\left[\begin{array}{ll}
6 & \ell_{1}
\end{array}\right]^{2}}{\left[\begin{array}{ll}
\ell_{1} & 6
\end{array}\right]\left[\begin{array}{ll}
6 & 1
\end{array}\right]\left[\begin{array}{ll}
1 & 2
\end{array}\right]\left[\begin{array}{ll}
2 & \ell_{2}
\end{array}\right]\left[\ell_{2} \ell_{1}\right.}\right] \frac{\left\langle 3 \ell_{2}\right\rangle^{2}\left\langle 3 \ell_{1}\right\rangle^{2}}{\left\langle\ell_{2} 3\right\rangle\langle 3 \quad 4\rangle\langle 45\rangle\left\langle 5 \ell_{1}\right\rangle\left\langle\ell_{1} \ell_{2}\right\rangle} \text {. }
\end{aligned}
$$

\subsection{The Integration of $C u t C_{34}$}

There are two terms in (3.2). Let us start with the first term. We wish to eliminate $\ell_{2}$ using the identities 8

$$
\frac{\left\langle\ell_{2}|1+2| 6\right]}{\left\langle\ell_{2} \ell_{1}\right\rangle}=-\frac{\left[\ell_{1}\left|P_{34} P_{12}\right| 6\right]}{P_{34}^{2}} \quad \text { and } \quad \frac{\left\langle 3 \ell_{2}\right\rangle}{\left\langle\ell_{1} \ell_{2}\right\rangle}=\frac{\left[4 \ell_{1}\right]}{\left[\begin{array}{ll}
4 & 3
\end{array}\right]}
$$

When this is done, we have

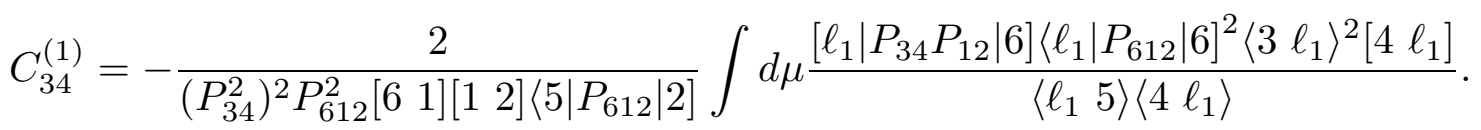

In the formula above, the measure is given by [18

$$
d \mu=\int_{0}^{+\infty} t d t\left\langle\ell_{1} d \ell_{1}\right\rangle\left[\ell_{1} d \ell_{1}\right] \delta\left(\left(P_{34}-\ell_{1}\right)^{2}\right)
$$

\footnotetext{
8 In our notation we have $P_{12}^{2}=\left\langle\begin{array}{lll}1 & 2\rangle\end{array}\left[\begin{array}{ll}1 & 2\end{array}\right]\right.$ and $2 p_{a} \cdot p_{b}=-\langle a|b| a]$.
} 
For simplicity, we write $\ell$ instead of $\ell_{1}$ from now on. We also represent $|\lambda\rangle$ by $|\ell\rangle$ and $\left.\mid \widetilde{\lambda}\right]$ by $\mid \ell]$. The cut integral is then

$$
\begin{aligned}
C_{34}^{(1)}=-\frac{2}{\left(P_{34}^{2}\right)^{2} P_{612}^{2}\left[\begin{array}{ll}
6 & 1
\end{array}\right]\left[\begin{array}{ll}
1 & 2
\end{array}\right]\left\langle 5\left|P_{612}\right| 2\right]} \int_{0}^{+\infty} & t d t\langle\ell d \ell\rangle[\ell d \ell] \delta\left(P_{34}^{2}+t\left\langle\ell\left|P_{34}\right| \ell\right]\right) \\
& \times \frac{t^{2}\left[\ell\left|P_{34} P_{12}\right| 6\right]\left\langle\ell\left|P_{612}\right| 6\right]^{2}\langle 3 \ell\rangle^{2}[4 \ell]}{\langle\ell 5\rangle\langle 4 \ell\rangle}
\end{aligned}
$$

where it is essential to note that an extra factor of $t^{2}$ shows up in the second line. The reason is that we have pulled out an overall $t$ factor when we write measure (3.4). That is to say, we have written [18,21]

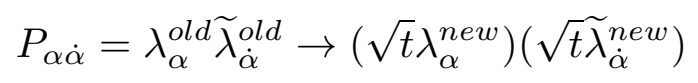

in the measure formula given by (3.4). Because of this scaling, the extra two pairs of $\lambda^{\text {old }}$ and $\widetilde{\lambda}^{\text {old }}$ in the numerator will give an extra $t^{2}$ after changing to the variables $\lambda^{n e w}, \widetilde{\lambda}^{\text {new }}$.

Since we are working in the dynamical region $P_{34}^{2}>0$, to find a nonzero result from the delta function we must have $\left\langle\ell\left|P_{34}\right| \ell\right]<0$. This is important when we integrate the delta function, because

$$
\delta(a x)=\frac{1}{|a|} \delta(x) .
$$

Now we can perform the $t$-integration to get

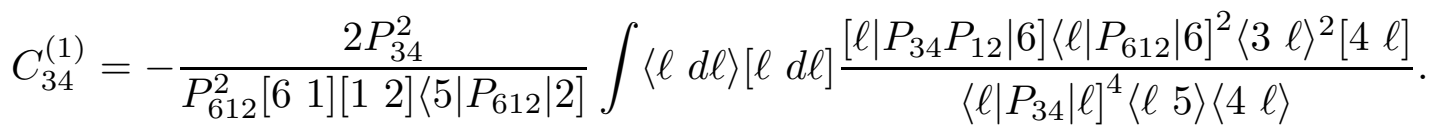

Notice that (3.7) is in the form of type (1) in (2.11), so in principle we can already apply (2.12) to do the integration. But by observing the identity

$$
\langle\ell 3\rangle\left[\ell\left|P_{34} P_{612}\right| 6\right]=-\left\langle\ell\left|P_{34}\right| \ell\right]\left\langle 3\left|P_{612}\right| 6\right]+\left\langle\ell\left|P_{612}\right| 6\right]\left\langle 3\left|P_{34}\right| \ell\right],
$$

we can split it into two terms that are much easier to integrate:

$$
C_{34}^{(1)}=C \int\langle\ell d \ell\rangle[\ell d \ell]\left(-\frac{\left\langle\ell\left|P_{612}\right| 6\right]^{2}\langle\ell 3\rangle[4 \ell]\left\langle 3\left|P_{612}\right| 6\right]}{\left\langle\ell\left|P_{34}\right| \ell\right]^{3}\langle\ell 5\rangle\langle 4 \ell\rangle}+\frac{\left\langle\ell\left|P_{612}\right| 6\right]^{3}\langle\ell 3\rangle[4 \ell]^{2}\langle 34\rangle}{\left\langle\ell\left|P_{34}\right| \ell\right]^{4}\langle\ell 5\rangle\langle 4 \ell\rangle}\right)
$$

where

$$
C=-\frac{2 P_{34}^{2}}{\left.P_{612}^{2}\left[\begin{array}{ll}
6 & 1][1
\end{array}\right]\left[\begin{array}{ll}
1 & 2
\end{array}\right] P_{612} \mid 2\right]}
$$


The reason for doing so is simple: we find that by a judicious choice of the auxiliary spinor $\eta$ in (2.12) we can reduce the integration to the special case (2.14) in which multiple poles have been canceled.

Using (2.14) for the first term of $C_{34}^{(1)}$, we get

$$
C_{34}^{(1 ; 1)}=-\frac{C}{2[34]} \int\langle\ell d \ell\rangle\left[d \ell \partial_{\ell}\right]\left[\frac{\left\langle\ell\left|P_{612}\right| 6\right]^{2}\left\langle 3\left|P_{612}\right| 6\right][4 \ell]^{2}}{\langle\ell 5\rangle\langle 4 \ell\rangle} \frac{1}{\left\langle\ell\left|P_{34}\right| \ell\right]^{2}}\right] .
$$

Now we can read off the contributions at the poles. Naively, there are two poles, $|\ell\rangle=|5\rangle$ and $|\ell\rangle=|4\rangle$. However, due to the factor of $\left[\begin{array}{ll}4 & \ell\end{array}\right]^{2}$ in the numerator, the residue at the second pole is zero. In the end we arrive at 9

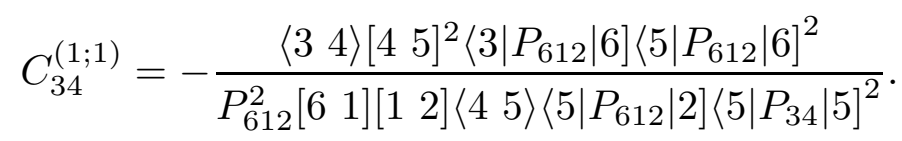

Similarly we can find the second term of $C_{34}^{(1)}$ and the second term of $C_{34}$. Putting it all together, we find that the coefficient of the bubble integral $I_{2: 2 ; 3}$ is

$$
\begin{aligned}
& c_{2: 2 ; 3}=-\frac{2\langle 23\rangle^{3}\left[\begin{array}{ll}
3 & 4
\end{array}\right]^{2}\left\langle 1\left|P_{561}\right| 2\right]^{3}}{3\left[\begin{array}{ll}
2 & 3
\end{array}\right]\left\langle 2\left|P_{34}\right| 2\right]^{3}\langle 56\rangle\langle 61\rangle P_{561}^{2}\left\langle 5\left|P_{561}\right| 2\right]}+\frac{\langle 23\rangle^{2}[34]\left\langle 1\left|P_{561}\right| 2\right]^{2}\left\langle 1\left|P_{561}\right| 4\right]}{\left[\begin{array}{lll}
2 & 3
\end{array}\right]\left\langle 2\left|P_{34}\right| 2\right]^{2}\langle 56\rangle\langle 61\rangle P_{561}^{2}\left\langle 5\left|P_{561}\right| 2\right]}
\end{aligned}
$$

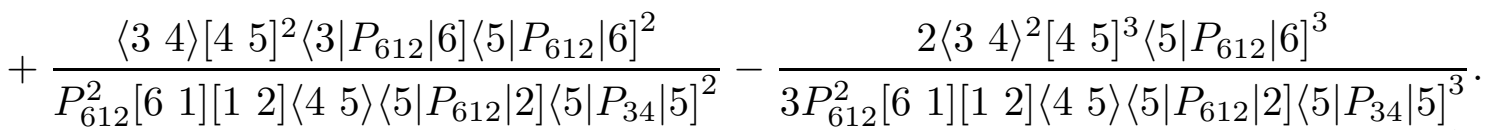

\subsection{The $C u t C_{612}$}

For the cut $C_{612}$ given by 3.3 we perform similar manipulations to reach

$$
\begin{array}{r}
C_{612}=\frac{2 P_{345}^{2}}{\left[\begin{array}{ll}
6 & 1
\end{array}\right]\left[\begin{array}{ll}
1 & 2
\end{array}\right]\langle 3 \quad 4\rangle\langle 45\rangle} \int\langle\ell d \ell\rangle[\ell d \ell]\left(\frac{\left\langle 3\left|P_{345}\right| 6\right]\left\langle\ell\left|P_{345}\right| 6\right]\langle 3 \ell\rangle^{2}}{\left\langle\ell\left|P_{345}\right| 2\right]\langle\ell 5\rangle} \frac{[6 \ell]}{\left\langle\ell\left|P_{345}\right| \ell\right]^{3}}\right. \\
\left.-\frac{P_{345}^{2}\left\langle\ell\left|P_{345}\right| 6\right]\langle 3 \ell\rangle^{3}}{\left\langle\ell\left|P_{345}\right| 2\right]\langle\ell 5\rangle} \frac{[6 \ell]^{2}}{\left\langle\ell\left|P_{345}\right| \ell\right]^{4}}\right) .
\end{array}
$$

9 Here we remark on signs in these calculations. There are several places to pay attention to signs. First, we need to write the pole in the right form $(\langle\ell a\rangle$ or $[\ell a])$ in order to apply the formulas of Section 2. Second, the contribution to the integral (as for example in the formulas (2.19) and (2.24) ) is the negative of the residue obtained by substituting the value of $\ell$ at the pole. However, to read out the coefficient of the corresponding bubble function we need to put another minus sign in front of the rational part of the cut integration, because of the minus sign in 2.5$)$. 
After doing the integration we find that the coefficient of the bubble integral $I_{2: 3 ; 6}$ is

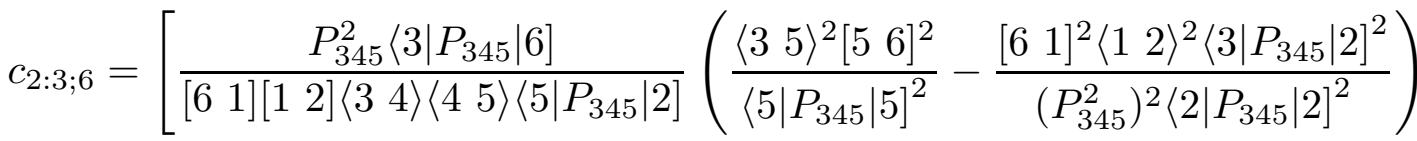

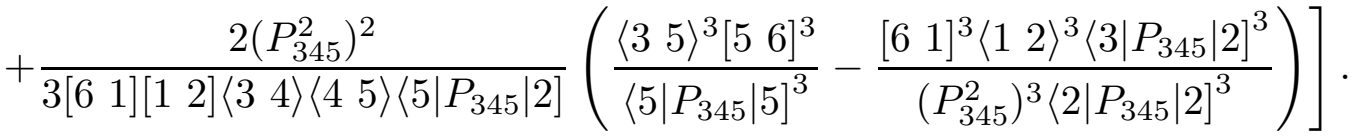

We have verified that the above results satisfy the singular behavior given by (2.3).

4. $A\left(1^{-}, 2^{+}, 3^{-}, 4^{+}, 5^{-}, 6^{+}\right)$

In this configuration, there are one-mass and two-mass-hard box functions, threemass triangle functions, and bubble functions. This amplitude is invariant under a $\mathbb{Z}_{6}$ symmetry generated by $\alpha: i \rightarrow i+1$ accompanied by conjugation. Because of this, we need to calculate just one coefficient for each type of function and act on it by $\alpha$ to obtain all the others. Representative box coefficients are given by [42]

$$
\begin{aligned}
c_{4: 2 ; 2}^{2 m} h & =\frac{2\left[\begin{array}{ll}
1 & 2
\end{array}\right]\langle 56\rangle\left\langle 5\left|P_{123}\right| 1\right]^{2}\left\langle 6\left|P_{123}\right| 2\right]^{2} P_{123}^{2}}{[23]\langle 4 \quad 5\rangle\left\langle 4\left|P_{123}\right| 1\right]\left\langle 6\left|P_{123}\right| 3\right]\left\langle 6\left|P_{123}\right| 1\right]^{4}} ; \\
c_{4 ; 4}^{1 m} & =\frac{2[1 \quad 2][23]\left\langle 5\left|P_{123}\right| 1\right]^{2}\left\langle 5\left|P_{123}\right| 3\right]^{2}}{\left[\begin{array}{ll}
1 & 3
\end{array}\right]^{4}\langle 4 \quad 5\rangle\langle 56\rangle\left\langle 4\left|P_{123}\right| 1\right]\left\langle 6\left|P_{123}\right| 3\right] P_{123}^{2}} .
\end{aligned}
$$

We need to choose just two representative integrals, one in a three-particle channel and one in a two-particle channel. We choose the following cuts.

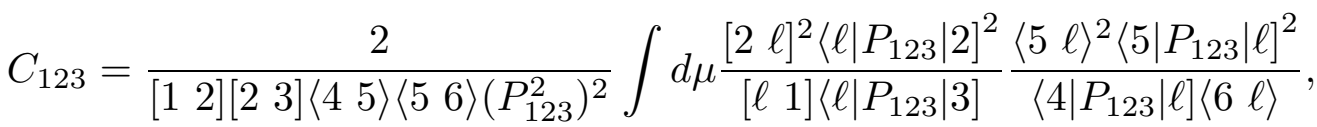

$$
\begin{aligned}
& C_{56}=2 \int d \mu \frac{\left[\ell_{1} 2\right]^{2}\left\langle 3 \ell_{2}\right\rangle^{2}\left\langle 3\left|P_{\ell_{1} 12}\right| 2\right]^{2}}{\left[\ell_{1} 1\right]\left[\begin{array}{ll}
1 & 2
\end{array}\right]\langle 34\rangle\left\langle 4 \ell_{2}\right\rangle P_{\ell_{1} 12}^{2}\left\langle 4\left|P_{\ell_{1} 12}\right| \ell_{1}\right]\left\langle\ell_{2}\left|P_{\ell_{1} 12}\right| 2\right]} \frac{\left\langle 5 \ell_{1}\right\rangle^{2}\left\langle 5 \ell_{2}\right\rangle^{2}}{\left\langle\ell_{2} 5\right\rangle\langle 56\rangle\left\langle 6 \ell_{1}\right\rangle\left\langle\ell_{1} \ell_{2}\right\rangle}
\end{aligned}
$$

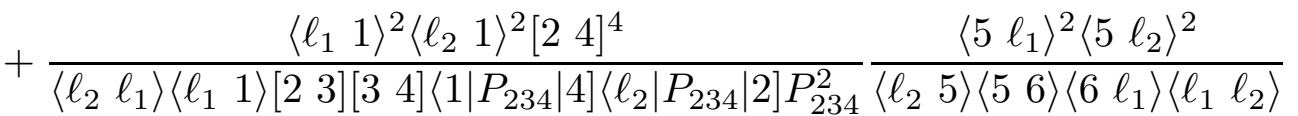

$$
\begin{aligned}
& +\frac{\left[4 \ell_{1}\right]^{2}\left[4 \ell_{2}\right]^{2}\langle 31\rangle^{4}}{\langle 12\rangle\langle 23\rangle\left[4 \ell_{2}\right]\left[\ell_{2} \ell_{1}\right] P_{123}^{2}\left\langle 3\left|P_{123}\right| \ell_{1}\right]\left\langle 1\left|P_{123}\right| 4\right]} \frac{\left\langle 5 \ell_{1}\right\rangle^{2}\left\langle 5 \ell_{2}\right\rangle^{2}}{\left\langle\ell_{2} 5\right\rangle\langle 56\rangle\left\langle 6 \ell_{1}\right\rangle\left\langle\ell_{1} \ell_{2}\right\rangle} .
\end{aligned}
$$




\subsection{Cut $C_{123}$}

We start with the cut $C_{123}$ because it does not contain three-mass triangle functions and should thus be easier to deal with. Since we know the box coefficients, we need only to extract the rational functions giving the bubble coefficients.

After the $t$-integration we get

$$
C_{123}=\frac{2\left(P_{123}^{2}\right)}{\left[\begin{array}{ll}
1 & 2
\end{array}\right]\left[\begin{array}{ll}
2 & 3
\end{array}\right]\langle 4\rangle\langle 56\rangle} \int\langle\ell d \ell\rangle[\ell d \ell] \frac{1}{\left\langle\ell\left|P_{123}\right| \ell\right]^{4}} \frac{[2 \ell]^{2}\left\langle\ell\left|P_{123}\right| 2\right]^{2}}{[\ell 1]\left\langle\ell\left|P_{123}\right| 3\right]} \frac{\langle 5 \ell\rangle^{2}\left\langle 5\left|P_{123}\right| \ell\right]^{2}}{\left\langle 4\left|P_{123}\right| \ell\right]\langle 6 \ell\rangle} .
$$

We look for singularities in $\mid \ell]$ in the denominator; we find two single poles $[\ell$ 1] and $\left\langle 4\left|P_{123}\right| \ell\right]$ and one quadruple pole $\left\langle\ell\left|P_{123}\right| \ell\right]$.

To demonstrate our general strategy, we give some details of the calculation. Since there are only two single poles, we can separate them by application of 2.10 to find

$C_{123}=C \int\langle\ell d \ell\rangle[\ell d \ell] \frac{1}{\left\langle\ell\left|P_{123}\right| \ell\right]^{4}} \frac{[2 \ell]^{2}\left\langle\ell\left|P_{123}\right| 2\right]^{2}}{\left\langle\ell\left|P_{123}\right| 3\right]} \frac{\langle 5 \ell\rangle^{2}\left\langle 5\left|P_{123}\right| \ell\right]}{\langle 6 \ell\rangle}\left(\frac{P_{123}^{2}\langle 54\rangle}{\left\langle 4\left|P_{123}\right| \ell\right]}+\frac{\left\langle 5\left|P_{123}\right| 1\right]}{[\ell 1]}\right)$

where

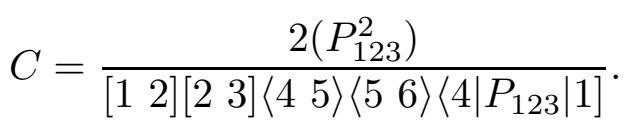

Next we split the simple pole from the quadruple pole by repeating (2.10). Finally we get

$$
C_{123}=C_{123}^{\text {rational }}+C_{123}^{\text {log }}
$$

where

$$
\begin{aligned}
& C_{123}^{\text {rational }}=-\frac{2\left(P_{123}^{2}\right)^{2}}{\left[\begin{array}{ll}
1 & 2
\end{array}\right]\left[\begin{array}{ll}
2 & 3
\end{array}\right]\langle 45\rangle\langle 56\rangle} \int\langle\ell d \ell\rangle[\ell d \ell] \frac{[2 \ell]^{2}}{\left\langle\ell\left|P_{123}\right| \ell\right]^{4}} \frac{\langle 5 \ell\rangle^{4}\left\langle\ell\left|P_{123}\right| 2\right]^{2}}{\langle\ell \quad 4\rangle\left\langle\ell\left|P_{123}\right| 1\right]\left\langle\ell\left|P_{123}\right| 3\right]\langle 6 \ell\rangle} \\
& +\frac{2\left(P_{123}^{2}\right)\langle 45\rangle^{2}}{\left[\begin{array}{ll}
1 & 2
\end{array}\right][23]\langle 45\rangle\langle 56\rangle\left\langle 4\left|P_{123}\right| 1\right]} \int\langle\ell d \ell\rangle[\ell d \ell] \frac{\left\langle\ell\left|P_{123}\right| 2\right]^{3}\langle 5 \ell\rangle^{2}}{\langle\ell\rangle^{2}\left\langle\ell\left|P_{123}\right| 3\right]\langle 6 \ell\rangle} \frac{[2 \ell]}{\left\langle\ell\left|P_{123}\right| \ell\right]^{3}} \\
& -\frac{2\langle 45\rangle^{2}}{\left[\begin{array}{ll}
1 & 2
\end{array}\right]\left[\begin{array}{ll}
2 & 3
\end{array}\right]\langle 45\rangle\langle 56\rangle\left\langle 4\left|P_{123}\right| 1\right]} \int\langle\ell d \ell\rangle[\ell d \ell] \frac{\left\langle\ell\left|P_{123}\right| 2\right]^{3}\langle 5 \ell\rangle^{2}\left\langle 4\left|P_{123}\right| 2\right]}{\langle\ell \quad\rangle^{3}\left\langle\ell\left|P_{123}\right| 3\right]\langle 6 \ell\rangle} \frac{1}{\left\langle\ell\left|P_{123}\right| \ell\right]^{2}} \\
& -\frac{2\left(P_{123}^{2}\right)\left\langle 5\left|P_{123}\right| 1\right]^{2}}{\left[\begin{array}{ll}
1 & 2
\end{array}[23]\langle 45\rangle\langle 56\rangle\left\langle 4\left|P_{123}\right| 1\right]\right.} \int\langle\ell d \ell\rangle[\ell d \ell] \frac{\left\langle\ell\left|P_{123}\right| 2\right]^{3}\langle 5 \ell\rangle^{2}}{\left\langle\ell\left|P_{123}\right| 1\right]^{2}\left\langle\ell\left|P_{123}\right| 3\right]\langle 6 \ell\rangle} \frac{[2 \ell]}{\left\langle\ell\left|P_{123}\right| \ell\right]^{3}}
\end{aligned}
$$

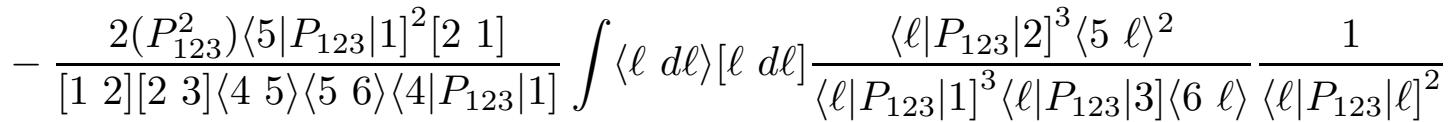


and

$$
\begin{aligned}
& C_{123}^{\log }= \\
& \frac{2\langle 45\rangle^{2}}{\left[\begin{array}{ll}
1 & 2
\end{array}\right]\left[\begin{array}{ll}
2 & 3
\end{array}\right]\langle 45\rangle\langle 56\rangle\left\langle 4\left|P_{123}\right| 1\right]} \int\langle\ell d \ell\rangle[\ell d \ell] \frac{\left\langle\ell\left|P_{123}\right| 2\right]^{2}\langle 5 \ell\rangle^{2}\left\langle 4\left|P_{123}\right| 2\right]^{2}}{\langle\ell} \frac{1\rangle^{3}\left\langle\ell\left|P_{123}\right| 3\right]\langle 6 \ell\rangle}{\left\langle\ell\left|P_{123}\right| \ell\right]\left\langle 4\left|P_{123}\right| \ell\right]} \\
& +\frac{2\left(P_{123}^{2}\right)\left\langle 5\left|P_{123}\right| 1\right]^{2}[21]^{2}}{\left[\begin{array}{ll}
1 & 2
\end{array}\right]\left[\begin{array}{ll}
2 & 3
\end{array}\right]\langle 45\rangle\langle 56\rangle\left\langle 4\left|P_{123}\right| 1\right]} \int\langle\ell d \ell\rangle[\ell d \ell] \frac{\left\langle\ell\left|P_{123}\right| 2\right]^{2}\langle 5 \ell\rangle^{2}}{\left\langle\ell\left|P_{123}\right| 1\right]^{3}\left\langle\ell\left|P_{123}\right| 3\right]\langle 6 \ell\rangle} \frac{1}{\left\langle\ell\left|P_{123}\right| \ell\right][\ell 1]} \text {. }
\end{aligned}
$$

The key feature of the above expansion is that each term in (4.3) is in the standard form given in (2.11), so we know how to deal with each one. Furthermore, as indicated by

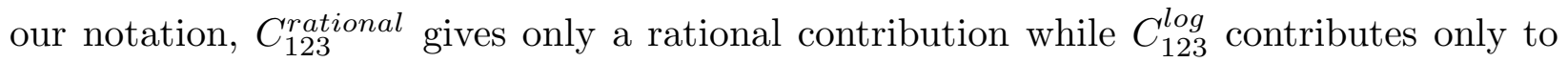
pure logarithmic terms (see Appendix B for a discussion of the latter), from which we can read the coefficients of corresponding one-mass and two-mass hard box functions. Since we have calculated these box functions by quadruple cuts, it can serve as an independent check of our method. However, we will not do it here.

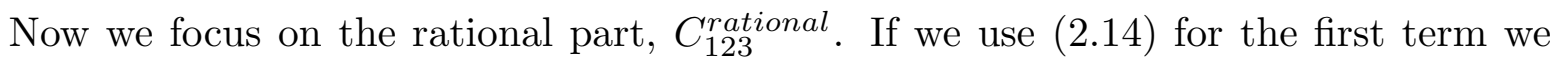
find that we get only a single pole, easily dealt with. The second and fourth terms have double poles which are new to us, so we will use them to demonstrate our general strategy as laid out in Section 2. However, before we proceed to a detailed treatment of double poles, we want to remark upon the third and fifth terms. Naively we have triple poles, but recall that in the relation

$$
[\ell d \ell] \frac{1}{\left\langle\ell\left|P_{123}\right| \ell\right]^{2}}=\left[d \ell \partial_{\ell}\right] \frac{[\eta \ell]}{\left\langle\ell\left|P_{123}\right| \ell\right]\left\langle\ell\left|P_{123}\right| \eta\right]}
$$

we have some freedom in the choice of $\mid \eta]$. If we choose $\mid \eta]=\mid 4]$ for the third term, the numerator factor $[4 \ell]$ will make the contribution from triple pole $\frac{1}{\langle\ell 4\rangle^{3}}$ to be zero. A similar manipulation can be done in fifth term by choosing $\left.\mid \eta]=\left|P_{123}\right| 1\right\rangle$.

Double Pole: The Second Term of $C_{123}^{\text {rational }}$

We can write the second term of $C_{123}^{\text {rational }}$ as

$$
C_{123}^{\text {rational } ; 2}=C \int\langle\ell d \ell\rangle\left[d \ell \partial_{\ell}\right]\left(\frac{\left\langle\ell\left|P_{123}\right| 2\right]^{3}\langle 5 \ell\rangle^{2}}{\langle\ell 4\rangle^{2}\left\langle\ell\left|P_{123}\right| 3\right]\langle 6 \ell\rangle} \frac{[2 \ell]^{2}}{\left\langle\ell\left|P_{123}\right| \ell\right]^{2}\left\langle\ell\left|P_{123}\right| 2\right]}\right)
$$

with

$$
C=\frac{\left(P_{123}^{2}\right)\langle 45\rangle}{\left[\begin{array}{ll}
1 & 2
\end{array}\right]\left[\begin{array}{ll}
2 & 3
\end{array}\right]\langle 56\rangle\left\langle 4\left|P_{123}\right| 1\right]} .
$$


There are several single poles but we will be concerned only with the double pole $\langle\ell 4\rangle$. To read out the residue at this double pole, we write the integrand as

$$
-C[2]^{2} \frac{1}{\langle\ell 4\rangle^{2}} \frac{\left\langle\ell\left|P_{123}\right| 2\right]^{3}\langle\ell 5\rangle^{2}}{\left\langle\ell\left|P_{123}\right| 3\right]\langle\ell 6\rangle\left\langle\ell\left|P_{123}\right| 4\right]^{2}\left\langle\ell\left|P_{123}\right| 2\right]},
$$

where we have made the replacement $\mid \ell]=\mid 4]$. Now it is in our standard form for a double pole, so we can use (2.18) and (2.19) to write down the answer as

$$
-C[24]^{2} P_{2}\left[|4\rangle, L_{1}^{\left(I I ; C_{123}\right)}, L_{2}^{\left(I I ; C_{123}\right)}\right],
$$

where we will make use of the following lists:

$$
\begin{aligned}
& \left.\left.L_{1}^{\left(I I ; C_{123}\right)}=\left\{|5\rangle,|5\rangle,\left|P_{123}\right| 2\right\rangle,\left|P_{123}\right| 2\right\rangle\right\}, \\
& \left.\left.\left.L_{2}^{\left(I I ; C_{123}\right)}=\left\{|6\rangle,\left|P_{123}\right| 3\right\rangle,\left|P_{123}\right| 4\right\rangle,\left|P_{123}\right| 4\right\rangle\right\} \\
& \left.L_{3}^{\left(I I ; C_{123}\right)}=\left\{|6\rangle,\left|P_{123}\right| 3\right\rangle,|1\rangle,|1\rangle\right\} .
\end{aligned}
$$

The Coefficient of Bubble $I_{2: 3 ; 1}$

Combining all these results, we find that the coefficient of the bubble integral $I_{2: 3 ; 1}$ is given by

$$
\begin{aligned}
& c_{2: 3 ; 1}= \\
& \frac{1}{\left[\begin{array}{ll}
1 & 2
\end{array}\right]\left[\begin{array}{ll}
2 & 3
\end{array}\right]\langle 4 \quad 5\rangle\langle 5 \quad 6\rangle}\left(-\frac{2\left(P_{123}^{2}\right)^{2}}{3} \sum_{i=1}^{4} \lim _{|\ell\rangle \rightarrow\left|\ell_{i}\right\rangle}\left\langle\ell \ell_{i}\right\rangle\left[\frac{[2 \ell]^{3}}{\left\langle\ell\left|P_{123}\right| \ell\right]^{3}} \frac{\langle 5 \ell\rangle^{4}\left\langle\ell\left|P_{123}\right| 2\right]}{\langle\ell 4\rangle\left\langle\ell\left|P_{123}\right| 1\right]\left\langle\ell\left|P_{123}\right| 3\right]\langle 6 \ell\rangle}\right]\right. \\
& +\frac{2\langle 45\rangle^{2}}{\left\langle 4\left|P_{123}\right| 1\right]} \sum_{i=1,2,6} \lim _{|\ell\rangle \rightarrow\left|\ell_{i}\right\rangle}\left\langle\ell \ell_{i}\right\rangle\left[-\frac{\left\langle\ell\left|P_{123}\right| 2\right]^{3}\langle 5 \ell\rangle^{2}\left\langle 4\left|P_{123}\right| 2\right]}{\langle\ell 4\rangle^{3}\left\langle\ell\left|P_{123}\right| 3\right]\langle 6 \ell\rangle} \frac{[4 \ell]}{\left\langle\ell\left|P_{123}\right| \ell\right]\left\langle\ell\left|P_{123}\right| 4\right]}\right] \\
& -\frac{2\left(P_{123}^{2}\right)\left\langle 5\left|P_{123}\right| 1\right]^{2}[21]}{\left\langle 4\left|P_{123}\right| 1\right]} \sum_{i=1,2,5} \lim _{|\ell\rangle \rightarrow\left|\ell_{i}\right\rangle}\left\langle\ell \ell_{i}\right\rangle\left[\frac{\left\langle\ell\left|P_{123}\right| 2\right]^{3}\langle 5 \ell\rangle^{2}}{\left\langle\ell\left|P_{123}\right| 1\right]^{3}\left\langle\ell\left|P_{123}\right| 3\right]\langle 6 \ell\rangle} \frac{\left\langle 1\left|P_{123}\right| \ell\right]}{\left\langle\ell\left|P_{123}\right| \ell\right] P_{123}^{2}\langle\ell 1\rangle}\right] \\
& +\sum_{i=1,2} \lim _{|\ell\rangle \rightarrow\left|\ell_{i}\right\rangle}\left\langle\ell \ell_{i}\right\rangle\left[\frac{\left(P_{123}^{2}\right)\langle 45\rangle^{2}}{\left\langle 4\left|P_{123}\right| 1\right]} \frac{\left\langle\ell\left|P_{123}\right| 2\right]^{2}\langle 5 \ell\rangle^{2}}{\langle\ell 4\rangle^{2}\left\langle\ell\left|P_{123}\right| 3\right]\langle 6 \ell\rangle} \frac{[2 \ell]^{2}}{\left\langle\ell\left|P_{123}\right| \ell\right]^{2}}\right. \\
& \left.-\frac{\left(P_{123}^{2}\right)\left\langle 5\left|P_{123}\right| 1\right]^{2}}{\left\langle 4\left|P_{123}\right| 1\right]} \frac{\left\langle\ell\left|P_{123}\right| 2\right]^{2}\langle 5 \ell\rangle^{2}}{\left\langle\ell\left|P_{123}\right| 1\right]^{2}\left\langle\ell\left|P_{123}\right| 3\right]\langle 6 \ell\rangle} \frac{[2 \ell]^{2}}{\left\langle\ell\left|P_{123}\right| \ell\right]^{2}}\right] \\
& +\frac{\left(P_{123}^{2}\right)\langle 45\rangle^{2}[24]^{2}}{\left\langle 4\left|P_{123}\right| 1\right]} P_{2}\left[|4\rangle, L_{1}^{\left(I I ; C_{123}\right)}, L_{2}^{\left(I I ; C_{123}\right)}\right] \\
& \left.\left.-\frac{\left\langle 5\left|P_{123}\right| 1\right]^{2}\langle 13\rangle^{2}[23]^{2}}{\left\langle 4\left|P_{123}\right| 1\right]\left(P_{123}^{2}\right)} P_{2}\left[\left|P_{123}\right| 1\right], L_{1}^{\left(I I ; C_{123}\right)}, L_{3}^{\left(I I ; C_{123}\right)}\right]\right)
\end{aligned}
$$


where the list $L_{i}^{\left(I I ; C_{123}\right)}$ has been given in $(4.6)$, while the various poles are given by

$$
\left.\left.\left.\left|\ell_{1}\right\rangle=\left|P_{123}\right| 3\right], \quad\left|\ell_{2}\right\rangle=|6\rangle, \quad\left|\ell_{3}\right\rangle=|4\rangle, \quad\left|\ell_{4}\right\rangle=\left|P_{123}\right| 1\right], \quad\left|\ell_{5}\right\rangle=|1\rangle, \quad\left|\ell_{6}\right\rangle=\left|P_{123}\right| 4\right] .
$$

Let us give a brief explanation for the result (4.7). The first three lines give single pole contributions of the first, the third and the fifth terms in (4.4). The fourth and fifth lines give single pole contributions of the second and the fourth terms in (4.4). The last two lines are double pole contributions of the second and the fourth terms in (4.4).

\section{2. $C u t C_{56}$}

Now we consider to the two-particle channel where the cut integral is given by (4.2). Of the three terms, only the first one will contribute to the three-mass triangle, so we leave it for last.

\section{The second term}

For the second term in (4.2) we achieve the following separation by our standard splitting process:

$$
\begin{aligned}
& C_{56}^{(2 r)}=\frac{2[24]^{4} P_{56}^{2}\langle 56\rangle}{\left[\begin{array}{ll}
2 & 3
\end{array}\right][34]\left\langle 1\left|P_{234}\right| 4\right] P_{234}^{2}} \int\langle\ell d \ell\rangle[\ell d \ell] \frac{\langle\ell 5\rangle\langle\ell 1\rangle^{3}[6 \ell]^{2}}{\left\langle\ell\left|P_{234}\right| 2\right]\langle\ell 6\rangle\left\langle\ell\left|P_{56}\right| \ell\right]^{4}} \\
& -\frac{2\left[\begin{array}{ll}
2 & 4
\end{array}\right]^{4} P_{56}^{2}\langle 61\rangle}{\left[\begin{array}{ll}
2 & 3
\end{array}\right]\left[\begin{array}{ll}
3 & 4
\end{array}\right]\left\langle 1\left|P_{234}\right| 4\right] P_{234}^{2}} \int\langle\ell d \ell\rangle[\ell d \ell] \frac{\langle\ell 5\rangle^{2}\langle\ell 1\rangle^{2}[6 \ell]}{\left\langle\ell\left|P_{234}\right| 2\right]\langle\ell 6\rangle^{2}\left\langle\ell\left|P_{56}\right| \ell\right]^{3}}
\end{aligned}
$$

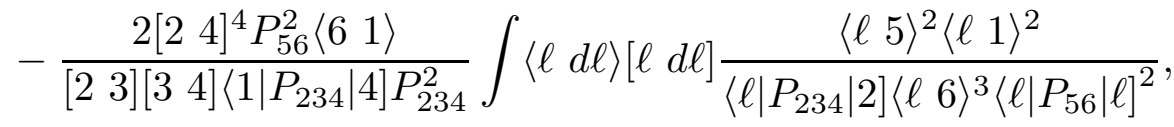

and

$$
C_{56}^{(2 l)}=\frac{2\left[\begin{array}{ll}
2 & 4
\end{array}\right]^{4} P_{56}^{2}\langle 61\rangle}{\left[\begin{array}{ll}
2 & 3
\end{array}\right]\left[\begin{array}{ll}
3 & 4
\end{array}\right]\left\langle 1\left|P_{234}\right| 4\right] P_{234}^{2}} \int\langle\ell d \ell\rangle[\ell d \ell] \frac{\langle\ell 5\rangle\langle\ell 1\rangle^{2}}{\left\langle\ell\left|P_{234}\right| 2\right]\langle\ell 6\rangle^{3}[5 \ell]\left\langle\ell\left|P_{56}\right| \ell\right]},
$$

where $r, l$ indicate rational and logarithmic parts, respectively.

It can be shown that in this case, with a judicious choice of the auxiliary spinor $\eta$, we can get rid of all multiple poles and are left with only single pole contributions. The contribution to the coefficient of the bubble $I_{2: 2 ; 5}$ is given by

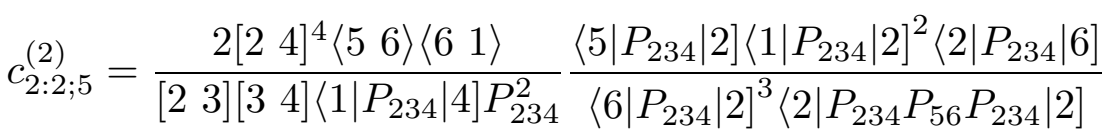

$$
\begin{aligned}
& +\frac{\left[\begin{array}{ll}
2 & 4
\end{array}\right]^{4}\langle 56\rangle\langle 61\rangle}{\left[\begin{array}{ll}
2 & 3
\end{array}\right]\left[\begin{array}{ll}
3 & 4
\end{array}\right]\left\langle 1\left|P_{234}\right| 4\right] P_{234}^{2}} \frac{\left\langle 1\left|P_{234}\right| 2\right]^{2}\left\langle 2\left|P_{234}\right| 6\right]^{2}\left\langle 5\left|P_{234}\right| 2\right]}{\left\langle 6\left|P_{234}\right| 2\right]^{2}\left\langle 2\left|P_{234} P_{56} P_{234}\right| 2\right]^{2}}
\end{aligned}
$$

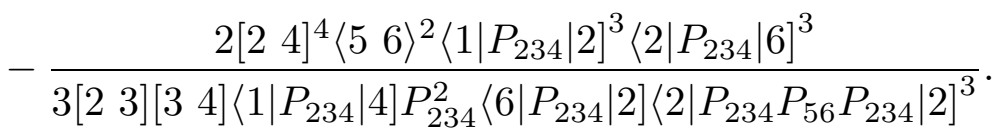


The third term

Now we split the third term of (4.2) into

$$
\begin{aligned}
C_{56}^{(3 r)} & =\frac{2\langle 31\rangle^{4} P_{56}^{2}[56]}{\langle 12\rangle\langle 23\rangle P_{123}^{2}\left\langle 1\left|P_{123}\right| 4\right]} \int\langle\ell d \ell\rangle[\ell d \ell] \frac{[4 \ell]^{3}[6 \ell]\langle\ell 5\rangle^{2}}{\left\langle 3\left|P_{123}\right| \ell\right][5 \ell]\left\langle\ell\left|P_{56}\right| \ell\right]^{4}} \\
& -\frac{2\langle 31\rangle^{4} P_{56}^{2}[45]}{\langle 12\rangle\langle 23\rangle P_{123}^{2}\left\langle 1\left|P_{123}\right| 4\right]} \int\langle\ell d \ell\rangle[\ell d \ell] \frac{[4 \quad \ell]^{2}[6 \ell]^{2}\langle\ell 5\rangle}{\left\langle 3\left|P_{123}\right| \ell\right][5 \ell]^{2}\left\langle\ell\left|P_{56}\right| \ell\right]^{3}} \\
& -\frac{2\langle 31\rangle^{4} P_{56}^{2}[45]}{\langle 12\rangle\langle 23\rangle P_{123}^{2}\left\langle 1\left|P_{123}\right| 4\right]} \int\langle\ell d \ell\rangle[\ell d \ell] \frac{[4 \ell]^{2}[6 \ell]^{2}}{\left\langle 3\left|P_{123}\right| \ell\right][5 \ell]^{3}\left\langle\ell\left|P_{56}\right| \ell\right]^{2}}
\end{aligned}
$$

and

$$
C_{56}^{(3 l)}=\frac{2\langle 31\rangle^{4} P_{56}^{2}[45]}{\langle 12\rangle\langle 23\rangle P_{123}^{2}\left\langle 1\left|P_{123}\right| 4\right]} \int\langle\ell d \ell\rangle[\ell d \ell] \frac{[4 \ell]^{2}[6 \ell]}{\left\langle 3\left|P_{123}\right| \ell\right][5 \ell]^{3}\langle\ell 6\rangle\left\langle\ell\left|P_{56}\right| \ell\right]}
$$

From here we read out the bubble coefficient part as

$$
\begin{aligned}
& c_{2: 2 ; 5}^{(3)}=-\frac{2\langle 13\rangle^{4}\left[\begin{array}{ll}
5 & 6
\end{array}\right]^{2}\left\langle 3\left|P_{123}\right| 4\right]^{3}\left\langle 5\left|P_{123}\right| 3\right]^{3}}{3\langle 12\rangle\langle 23\rangle P_{123}^{2}\left\langle 1\left|P_{123}\right| 4\right]\left\langle 3\left|P_{123}\right| 5\right]\left\langle 3\left|P_{123} P_{56} P_{123}\right| 3\right]^{3}}
\end{aligned}
$$

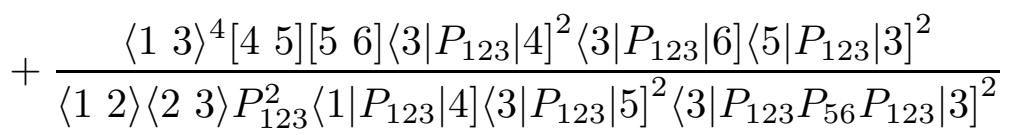

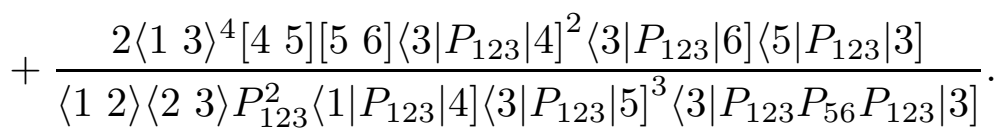

The first term

Now we move to the first term in (4.2). After doing the $t$-integration and setting $\ell=\ell_{1}$, we get

$$
\begin{aligned}
& C_{56}^{(1)}=-\frac{2}{\left[\begin{array}{ll}
1 & 2
\end{array}\right]\langle 34\rangle P_{56}^{2}} \int\langle\ell d \ell\rangle[\ell d \ell] \frac{\left(P_{56}^{2}\right)^{2}}{\left\langle\ell\left|P_{56}\right| \ell\right]^{3}} \frac{[\ell]^{2}\left\langle 3\left|P_{56}\right| \ell\right]^{2}\langle 5 \ell\rangle^{2}[6 \ell]}{\left[\ell|4| P_{12} \mid \ell\right]\left\langle 4\left|P_{56}\right| \ell\right]\left[2\left|P_{5612} P_{56}\right| \ell\right]\langle 6 \ell\rangle} \\
& \times \frac{\left\langle 3\left|P_{12}\right| 2\right]^{2}-2 \frac{P_{56}^{2}}{\left\langle\ell\left|P_{56}\right| \ell\right]}\left\langle 3\left|P_{12}\right| 2\right]\langle 3 \ell\rangle[\ell 2]+\frac{\left(P_{56}^{2}\right)^{2}}{\left\langle\ell\left|P_{56}\right| \ell\right]^{2}}\langle 3 \ell\rangle^{2}[\ell 2]^{2}}{P_{12}^{2}+\frac{P_{56}^{2}\left\langle\ell\left|P_{12}\right| \ell\right]}{\left\langle\ell\left|P_{56}\right| \ell\right]}} .
\end{aligned}
$$

Defining

$$
Q=P_{12}+\frac{P_{12}^{2}}{P_{56}^{2}} P_{56}
$$


we get

$$
\begin{aligned}
& C_{56}^{(1)}=-\frac{2}{\left[\begin{array}{ll}
1 & 2
\end{array}\right]\langle 34\rangle} \int\langle\ell d \ell\rangle[\ell d \ell] \frac{1}{\left\langle\ell\left|P_{56}\right| \ell\right]^{3}} \frac{[\ell 2]^{2}\left\langle 3\left|P_{56}\right| \ell\right]^{2}\langle 5 \ell\rangle^{2}[6 \ell]}{[\ell 1]\left\langle 4\left|P_{12}\right| \ell\right]\left\langle 4\left|P_{56}\right| \ell\right]\left[2\left|P_{5612} P_{56}\right| \ell\right]\langle 6 \ell\rangle} \\
& \left(\frac{\left\langle 3\left|P_{12}\right| 2\right]^{2}\left\langle\ell\left|P_{56}\right| \ell\right]}{\langle\ell|Q| \ell]}-\frac{2 P_{56}^{2}\left\langle 3\left|P_{12}\right| 2\right]\langle 3 \ell\rangle[\ell 2]}{\langle\ell|Q| \ell]}+\frac{\left(P_{56}^{2}\right)^{2}\langle 3 \ell\rangle^{2}[\ell 2]^{2}}{\left\langle\ell\left|P_{56}\right| \ell\right]\langle\ell|Q| \ell]}\right) \text {. }
\end{aligned}
$$

To simplify the calculation further we define

$$
g(\widetilde{\ell}) \equiv-\frac{[\ell 6][\ell 2]^{2}\left[\ell\left|P_{56}\right| 3\right\rangle^{2}}{[\ell \quad 1]\left[\ell\left|P_{12}\right| 4\right\rangle\left[\ell\left|P_{56}\right| 4\right\rangle\left[\ell\left|P_{56} P_{34}\right| 2\right]}
$$

The tilde in $g(\widetilde{\ell})$ indicates that this function is antiholomorphic.

Now we can use our standard splitting method to split each of them to reach the form given in (2.11). The result is

$$
C_{56}^{(1)}=C_{56}^{(1 r)}+C_{56}^{(1 l)}+C_{56}^{(1 ; 3 m)}
$$

where $1 r, 1 l, 3 m$ respectively indicate rational contributions, logarithmic contributions for box functions and logarithmic contributions for three-mass triangle functions. Since we do not compute box coefficients from double cuts, we do not record them here, but give only the other two parts as: 


$$
\begin{aligned}
& C_{56}^{(1 r)}=-\frac{2\left(P_{56}^{2}\right)^{2}}{[1 \quad 2]\langle 34\rangle} \int\langle\ell d \ell\rangle[\ell d \ell] \frac{g(\widetilde{\ell})[\ell 2]^{2}\left\langle 3\left|P_{56}\right| \ell\right]^{2}}{\left\langle 6\left|P_{56}\right| \ell\right]\left[\ell\left|P_{56} Q\right| \ell\right]} \frac{\langle 5 \ell\rangle^{2}}{\left\langle\ell\left|P_{56}\right| \ell\right]^{4}} \\
& +\frac{2\left(P_{56}^{2}\right)^{2}}{[112]\langle 34\rangle} \int\langle\ell d \ell\rangle[\ell d \ell] \frac{g(\widetilde{\ell})[\ell 2]^{2}\langle 36\rangle^{2}\left\langle 5\left|P_{56}\right| \ell\right]}{\langle 6|Q| \ell]\left\langle 6\left|P_{56}\right| \ell\right]^{2}} \frac{\langle 5 \ell\rangle}{\left\langle\ell\left|P_{56}\right| \ell\right]^{3}} \\
& -\frac{2\left(P_{56}^{2}\right)^{2}}{\left[11^{2}\right]\langle 34\rangle} \int\langle\ell d \ell\rangle[\ell d \ell] \frac{g(\widetilde{\ell})[\ell 2]^{2}\langle 3|Q| \ell]^{2}\left\langle 5\left|P_{56}\right| \ell\right]}{\langle 6|Q| \ell]\left[\ell\left|P_{56} Q\right| \ell\right]^{2}} \frac{\langle 5 \ell\rangle}{\left\langle\ell\left|P_{56}\right| \ell\right]^{3}} \\
& +\frac{4 P_{56}^{2}\langle 13\rangle}{\langle 34\rangle} \int\langle\ell d \ell\rangle[\ell d \ell] \frac{g(\widetilde{\ell})[\ell 2][6 \ell]\left\langle 3\left|P_{56}\right| \ell\right]}{[5 \ell]\left[\ell\left|P_{56} Q\right| \ell\right]} \frac{\langle 5 \ell\rangle}{\left\langle\ell\left|P_{56}\right| \ell\right]^{3}} \\
& +\frac{4 P_{56}^{2}\langle 13\rangle}{\langle 34\rangle} \int\langle\ell d \ell\rangle[\ell d \ell] \frac{g(\widetilde{\ell})[\ell 2][6 \ell]^{2}\langle 65\rangle\langle 3|Q| \ell]}{[\ell 5]\left[\ell\left|P_{56} Q\right| \ell\right]^{2}} \frac{1}{\left\langle\ell\left|P_{56}\right| \ell\right]^{2}} \\
& -\frac{4 P_{56}^{2}\langle 13\rangle}{\langle 34\rangle} \int\langle\ell d \ell\rangle[\ell d \ell] \frac{g(\widetilde{\ell})[\ell 2][6 \ell]\left\langle 3\left|P_{56}\right| \ell\right]}{[\ell 5]^{2}\left[\ell\left|P_{56} Q\right| \ell\right]} \frac{1}{\left\langle\ell\left|P_{56}\right| \ell\right]^{2}} \\
& -\frac{2\langle 13\rangle^{2}\left[\begin{array}{ll}
1 & 2
\end{array}\right]}{\langle 34\rangle} \int\langle\ell d \ell\rangle[\ell d \ell] g(\widetilde{\ell}) \frac{g(\widetilde{\ell})\langle 56\rangle[6 \ell]^{2}}{[5 \ell]\left[\ell\left|P_{56} Q\right| \ell\right]} \frac{1}{\left\langle\ell\left|P_{56}\right| \ell\right]^{2}} \\
& +\frac{2\left(P_{56}^{2}\right)^{2}}{[12]\langle 34\rangle} \int\langle\ell d \ell\rangle[\ell d \ell] \frac{g(\widetilde{\ell})[\ell 2]^{2}\langle 36\rangle^{2}\left\langle 5\left|P_{56}\right| \ell\right]\langle 56\rangle}{\langle 6|Q| \ell]\left\langle 6\left|P_{56}\right| \ell\right]^{3}} \frac{1}{\left\langle\ell\left|P_{56}\right| \ell\right]^{2}}
\end{aligned}
$$

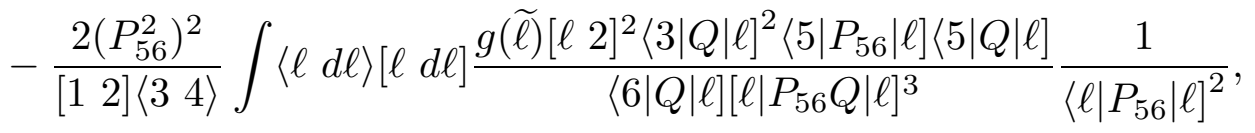

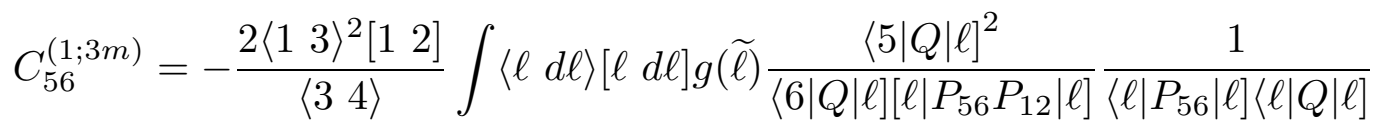

$$
\begin{aligned}
& +\frac{2\left(P_{56}^{2}\right)^{2}}{[12]\langle 34\rangle} \int\langle\ell d \ell\rangle[\ell d \ell] g(\widetilde{\ell})[\ell 2]^{2} \frac{\langle 3|Q| \ell]^{2}\langle 5|Q| \ell]^{2}}{\langle 6|Q| \ell]\left[\ell\left|P_{56} P_{12}\right| \ell\right]^{3}} \frac{1}{\left\langle\ell\left|P_{56}\right| \ell\right]\langle\ell|Q| \ell]} \\
& +\frac{4 P_{56}^{2}\langle 13\rangle}{\langle 34\rangle} \int\langle\ell d \ell\rangle[\ell d \ell] g(\widetilde{\ell})[\ell 2] \frac{\langle 3|Q| \ell]\langle 5|Q| \ell]^{2}}{\langle 6|Q| \ell]\left[\ell\left|P_{56} P_{12}\right| \ell\right]^{2}} \frac{1}{\left\langle\ell\left|P_{56}\right| \ell\right]\langle\ell|Q| \ell]} .
\end{aligned}
$$

Now we will discuss them one by one. But before doing that we need to know how to deal with poles from $\left[\ell\left|P_{56} Q\right| \ell\right]$.

Dealing with $\left[\ell\left|P_{56} Q\right| \ell\right]$ :

Now we need to deal with the factor $\left[\ell\left|P_{56} Q\right| \ell\right]$ in the denominator. We expand it as

$$
\mid \ell]=\mid a]+x \mid b]
$$


where $a, b$ are two arbitrary massless spinors. With this substitution we find

$$
\begin{aligned}
0 & =\left[a\left|P_{56} Q\right| a\right]+x\left(\left[a\left|P_{56} Q\right| b\right]+\left[b\left|P_{56} Q\right| a\right]\right)+x^{2}\left[b\left|P_{56} Q\right| b\right] \\
x_{ \pm} & =\frac{-\left(\left[a\left|P_{56} Q\right| b\right]+\left[b\left|P_{56} Q\right| a\right]\right) \pm[a b] \sqrt{\Delta_{3 m}}}{2\left[b\left|P_{56} Q\right| b\right]} \\
\Delta_{3 m} & =\left(P_{12}^{2}\right)^{2}+\left(P_{34}^{2}\right)^{2}+\left(P_{56}^{2}\right)^{2}-2 P_{12}^{2} P_{34}^{2}-2 P_{12}^{2} P_{56}^{2}-2 P_{34}^{2} P_{56}^{2}
\end{aligned}
$$

Now we can write the factor as

$$
\begin{aligned}
{\left[\ell \eta_{1}\right]\left[\ell \eta_{2}\right] } & =[\ell a]^{2}+\left(x_{+}+x_{-}\right)[\ell a][\ell b]+x_{+} x_{-}[\ell b]^{2} \\
& =\frac{[a b]^{2}\left[\ell\left|P_{56} Q\right| \ell\right]}{\left[b\left|P_{56} Q\right| b\right]}
\end{aligned}
$$

where $\left.\left.\left.\mid \eta_{1}\right]=\mid a\right]+x_{+} \mid b\right]$ and $\left.\left.\left.\mid \eta_{2}\right]=\mid a\right]+x_{-} \mid b\right]$. In other words, we write

$$
\left[\ell\left|P_{56} Q\right| \ell\right]=\left[\ell \eta_{1}\right]\left[\ell \eta_{2}\right] \frac{\left[b\left|P_{56} Q\right| b\right]}{[a b]^{2}},
$$

where

$$
\left.\left.\left.\left.\left.\left.\mid \eta_{1}\right]=\mid a\right]+x_{+} \mid b\right], \quad \mid \eta_{2}\right]=\mid a\right]+x_{-} \mid b\right], \quad x_{ \pm}=\frac{-\left(\left[a\left|P_{56} Q\right| b\right]+\left[b\left|P_{56} Q\right| a\right]\right) \pm[a b] \sqrt{\Delta_{3 m}}}{2\left[b\left|P_{56} Q\right| b\right]} .
$$

\section{The Rational Part:}

Now we discuss the rational part, $C_{56}^{(1 r)}$, given in (4.15). Upon a few moments' study, we see that among these nine terms, only the ninth one has a triple pole and only the third and the fifth have double poles. All of the remaining six terms have only single poles. For example, although the second term has the pole $\frac{1}{\langle 65\rangle^{2}[5 \ell]^{2}}$, the factor of $\langle 5 \ell\rangle$ in the numerator sets its contribution to zero. Also, the first term in (4.15) has a factor of $\frac{1}{[6 \ell]}$, but $g(\tilde{\ell})$ has the same factor in the numerator, so it is not a pole.

To read out the rational contributions we define the following three functions:

$$
\begin{aligned}
& A_{1}=\frac{2\left(P_{56}^{2}\right)^{2}[a b]^{2}}{3[1 \quad 2]\langle 34\rangle\langle 56\rangle^{2}\left[b\left|P_{56} Q\right| b\right]} \frac{g(\widetilde{\ell})[\ell 2]^{2}\left\langle 3\left|P_{56}\right| \ell\right]^{2}\langle 5 \ell\rangle^{3}}{[5 \ell]\left[\ell \eta_{1}\right]\left[\ell \eta_{2}\right][6 \ell]\left\langle\ell\left|P_{56}\right| \ell\right]^{3}}
\end{aligned}
$$

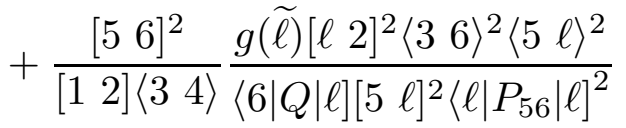

$$
\begin{aligned}
& +\frac{2 P_{56}^{2}\langle 13\rangle[a b]^{2}}{\langle 34\rangle\langle 56\rangle\left[b\left|P_{56} Q\right| b\right]} \frac{g(\tilde{\ell})[\ell 2]\left\langle 3\left|P_{56}\right| \ell\right]\langle 5 \ell\rangle^{2}}{[5 \ell]\left[\ell \eta_{1}\right]\left[\ell \eta_{2}\right]\left\langle\ell\left|P_{56}\right| \ell\right]^{2}} \\
& -\frac{4 P_{56}^{2}\langle 13\rangle[a b]^{2}}{\langle 34\rangle\langle 56\rangle\left[b\left|P_{56} Q\right| b\right]} \frac{g(\tilde{\ell})[\ell 2]\left\langle 3\left|P_{56}\right| \ell\right]\langle 5 \ell\rangle}{[\ell]^{2}\left[\ell \eta_{1}\right]\left[\ell \eta_{2}\right]\left\langle\ell\left|P_{56}\right| \ell\right]}
\end{aligned}
$$

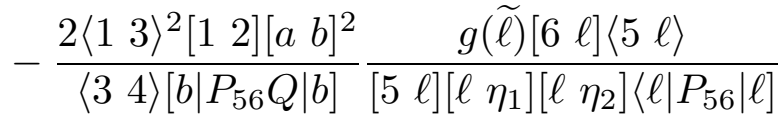

$$
\begin{aligned}
& -\frac{2\left(P_{56}^{2}\right)^{2}\langle 36\rangle^{2}}{[12]\langle 34\rangle\langle 56\rangle^{2}} \frac{g(\tilde{\ell})[\ell 2]^{2}\langle 5 \ell\rangle}{\langle 6|Q| \ell][5 \ell]^{3}\left\langle\ell\left|P_{56}\right| \ell\right]}
\end{aligned}
$$




$$
\begin{aligned}
A_{2}= & -\frac{\left(P_{56}^{2}\right)^{2}[a b]^{4}}{[1 \quad 2]\langle 34\rangle\left[b\left|P_{56} Q\right| b\right]^{2}} \frac{g(\widetilde{\ell})[\ell 2]^{2}\langle 3|Q| \ell]^{2}\langle 5 \ell\rangle^{2}}{\langle 6|Q| \ell]\left[\ell \eta_{1}\right]^{2}\left[\ell \eta_{2}\right]^{2}\left\langle\ell\left|P_{56}\right| \ell\right]^{2}} \\
& -\frac{4 P_{56}^{2}\langle 13\rangle[a b]^{4}}{\langle 34\rangle\left[b\left|P_{56} Q\right| b\right]^{2}} \frac{g(\widetilde{\ell})[\ell 2][6 \ell]\langle 3|Q| \ell]\langle 5 \ell\rangle}{[\ell 5]\left[\ell \eta_{1}\right]^{2}\left[\ell \eta_{2}\right]^{2}\left\langle\ell\left|P_{56}\right| \ell\right]} \\
A_{3}=- & \frac{2\left(P_{56}^{2}\right)^{2}[a b]^{6}}{[1 \quad 2]\langle 34\rangle\left[b\left|P_{56} Q\right| b\right]^{3}} \frac{g\left(\widetilde{\ell}[\ell 2]^{2}\langle 3|Q| \ell]^{2}\left\langle 5\left|P_{56}\right| \ell\right]\langle 5|Q| \ell]\left\langle\eta_{1} \ell\right\rangle\right.}{\langle 6|Q| \ell]\left\langle\eta_{1}\left|P_{56}\right| \ell\right]\left[\ell \eta_{1}\right]^{3}\left[\ell \eta_{2}\right]^{3}\left\langle\ell\left|P_{56}\right| \ell\right]}
\end{aligned}
$$

The function $A_{1}$ is the collection of the first, second, fourth, sixth, seventh and eighth terms after writing them in the form of $\left\langle d \ell \partial_{\ell}\right\rangle(\bullet)$. Similarly, $A_{2}$ is the collection of the third and fifth terms, while $A_{3}$ is the ninth term, after writing them in the form $\left\langle d \ell \partial_{\ell}\right\rangle(\bullet)$. Unlike in $A_{1}$, we have double poles in $A_{2}$ and triple poles in $A_{3}$, so we need to separate these contributions. One feature $A_{3}$ is that we have chosen the auxiliary spinor carefully to cancel one of the two triple poles (notice the factor $\left\langle\eta_{1} \ell\right\rangle$ in numerator).

First, $A_{1}$ gives following contribution to the bubble coefficient:

$$
c_{2: 2 ; 5}^{1 r ; A 1}=\sum_{i=1}^{7} \lim _{\ell \rightarrow \ell_{i}}\left(\left[\ell \ell_{i}\right] A_{1}\right)
$$

where

$\left.\left.\left.\left.\left.\left.\left.\left.\left.\left.\left.\left.\left.\left.\mid \ell_{1}\right]=\mid 1\right], \mid \ell_{2}\right]=\left|P_{12}\right| 4\right\rangle, \mid \ell_{3}\right]=\left|P_{56}\right| 4\right\rangle, \quad \mid \ell_{4}\right]=\left|P_{56} P_{5612}\right| 2\right], \quad \mid \ell_{5}\right]=|Q| 6\right\rangle, \mid \ell_{6}\right]=\mid \eta_{1}\right], \quad \mid \ell_{7}\right]=\mid \eta_{2}\right]$

$A_{2}$ gives following single pole contribution

$$
c_{2: 2 ; 5}^{1 r ; A 2-1}=\sum_{i=1}^{5} \lim _{\ell \rightarrow \ell_{i}}\left(\left[\ell \ell_{i}\right] A_{2}\right)
$$

and double pole contribution

$$
\begin{aligned}
& c_{2: 2 ; 5}^{1 r ; A 2-2}= \\
& \left.\left.\left.\left.\frac{\left(P_{56}^{2}\right)^{2}[a b]^{4}\left\langle 5 \eta_{1}\right\rangle^{2}}{\left[\begin{array}{ll}
1 & 2]\langle 3
\end{array}\right]\left[b\left|P_{56} Q\right| b\right]^{2}} P_{2}\left[\mid \eta_{1}\right], L_{1}, L_{2}\right]-\frac{4 P_{56}^{2}\langle 13\rangle[a b]^{4}\left\langle 5 \eta_{1}\right\rangle}{\langle 34\rangle\left[b\left|P_{56} Q\right| b\right]^{2}} P_{2}\left[\mid \eta_{1}\right], L_{3}, L_{4}\right]+\left\{\mid \eta_{1}\right] \leftrightarrow \mid \eta_{2}\right]\right\}
\end{aligned}
$$

where these lists are

$$
\begin{aligned}
& \left.\left.\left.\left.\left.\left.\left.\left.\left.L_{1}=\{\mid 6], \mid 2\right], \mid 2\right],\left|P_{56}\right| 3\right\rangle,\left|P_{56}\right| 3\right\rangle, \mid 2\right], \mid 2\right],|Q| 3\right\rangle,|Q| 3\right\rangle\right\} \\
& \left.\left.\left.\left.\left.\left.\left.\left.\left.L_{2}=\{\mid 1],\left|P_{12}\right| 4\right\rangle,\left|P_{56}\right| 4\right\rangle,\left|P_{56} P_{34}\right| 2\right],|Q| 6\right\rangle, \mid \eta_{2}\right], \mid \eta_{2}\right],\left|P_{56}\right| \eta_{1}\right\rangle,\left|P_{56}\right| \eta_{1}\right\rangle\right\} \\
& \left.\left.\left.\left.\left.\left.\left.\left.L_{3}=\{\mid 6], \mid 2\right], \mid 2\right],\left|P_{56}\right| 3\right\rangle,\left|P_{56}\right| 3\right\rangle, \mid 2\right], \mid 6\right],|Q| 3\right\rangle\right\} \\
& \left.\left.\left.\left.\left.\left.\left.\left.L_{4}=\{\mid 1],\left|P_{12}\right| 4\right\rangle,\left|P_{56}\right| 4\right\rangle,\left|P_{56} P_{34}\right| 2\right], \mid 5\right], \mid \eta_{2}\right], \mid \eta_{2}\right],\left|P_{56}\right| \eta_{1}\right\rangle\right\}
\end{aligned}
$$


and the function $P_{2}$ is given in (2.19).

For $A_{3}$, the single pole contribution is

$$
c_{2: 2 ; 5}^{1 r ; A 3-1}=\sum_{i=1}^{5} \lim _{\ell \rightarrow \ell_{i}}\left(\left[\ell \ell_{i}\right] A_{3}\right)+\lim _{\left.\ell \rightarrow\left|P_{56}\right| \eta_{1}\right\rangle}\left(\left[\ell\left|P_{56}\right| \eta_{1}\right\rangle A_{3}\right),
$$

and the triple pole contribution is

$$
\left.c_{2: 2 ; 5}^{1 r ; A 3-2}=-\frac{2\left(P_{56}^{2}\right)^{2}[a b]^{6}\langle 56\rangle\left\langle\eta_{1} \eta_{2}\right\rangle}{\left[\begin{array}{ll}
1 & 2]\langle 3
\end{array}\right]\left\langle b\left|P_{56} Q\right| b\right]^{3}} P_{3}\left[\mid \eta_{2}\right], L_{5}, L_{6}\right]
$$

with

$$
\begin{aligned}
& \left.\left.\left.\left.\left.\left.\left.\left.\left.\left.\left.L_{5}=\{\mid 6], \mid 2\right], \mid 2\right],\left|P_{56}\right| 3\right\rangle,\left|P_{56}\right| 3\right\rangle, \mid 2\right], \mid 2\right],|Q| 3\right\rangle,|Q| 3\right\rangle, \mid 6\right],|Q| 5\right\rangle\right\} \\
& \left.\left.\left.\left.\left.\left.\left.\left.\left.L_{6}=\{\mid 1],\left|P_{12}\right| 4\right\rangle,\left|P_{56}\right| 4\right\rangle,\left|P_{56} P_{34}\right| 2\right],|Q| 6|\rangle, \mid \eta_{1}\right], \mid \eta_{1}\right], \mid \eta_{1}\right],\left|P_{56}\right| \eta_{1}\right\rangle,\left|P_{56}\right| \eta_{1}\right\rangle\right\},
\end{aligned}
$$

and the function $P_{3}$ is given in (2.24).

Putting it all together, we find that the coefficient $c_{2: 2 ; 5}$ is given by the sum of (4.9), (4.11), 4.23), (4.24), (4.25), (4.27) and (4.28).

\section{The coefficient of triangle $I_{3: 2,2 ; 1}$ :}

To read off the coefficient of three-mass triangle function $I_{3: 2,2 ; 1}$ from (4.16), we need to be careful about different poles. As discussed carefully in Appendix B, poles from the factor $\left[\ell\left|P_{56} Q\right| \ell\right]$ are special. In this example, the poles from factors other than $\left[\ell\left|P_{56} Q\right| \ell\right]$ are all single poles, and their contributions are given by

$$
\begin{aligned}
c_{3: 2,2 ; 1}^{(1)} & =\sum_{i=1}^{6} \lim _{\left.\mid \ell] \rightarrow \mid \ell_{i}\right]}\left[\ell \ell_{i}\right] \frac{g(\widetilde{\ell})}{[\ell \widetilde{\eta}]} R_{1}\left[\ell, \widetilde{\eta}, P_{56}, Q\right]\left[-\frac{2\langle 13\rangle^{2}[12]}{\langle 34\rangle} \frac{\langle 5|Q| \ell]^{2}}{\langle 6|Q| \ell]\left[\ell\left|P_{56} P_{12}\right| \ell\right]}\right. \\
& \left.+\frac{2\left(P_{56}^{2}\right)^{2}}{[12]\langle 34\rangle} \frac{[\ell 2]^{2}\langle 3|Q| \ell]^{2}\langle 5|Q| \ell]^{2}}{\langle 6|Q| \ell]\left[\ell\left|P_{56} P_{12}\right| \ell\right]^{3}}+\frac{4 P_{56}^{2}\langle 13\rangle}{\langle 34\rangle} \frac{[\ell 2]\langle 3|Q| \ell]\langle 5|Q| \ell]^{2}}{\langle 6|Q| \ell]\left[\ell\left|P_{56} P_{12}\right| \ell\right]^{2}}\right]
\end{aligned}
$$

with the following poles:

$$
\begin{aligned}
& \left.\left.\left.\left.\left.\left.\left.\left.\left.\left.\left.\left.\mid \ell_{1}\right]=\mid 1\right], \quad \mid \ell_{2}\right]=\left|P_{12}\right| 4\right\rangle, \quad \mid \ell_{3}\right]=\left|P_{56}\right| 4\right\rangle, \quad \mid \ell_{4}\right]=\left|P_{56} P_{5612}\right| 2\right], \quad \mid \ell_{5}\right]=|Q| 6\right\rangle, \quad \mid \ell_{6}\right]=\mid \widetilde{\eta}\right] \\
& \left.\left.\left.\left.\mid \ell_{7}\right]=\mid \eta_{1}\right], \quad \mid \ell_{8}\right]=\mid \eta_{2}\right]
\end{aligned}
$$

Now consider the contributions from the poles from $\left[\ell\left|P_{56} Q\right| \ell\right]$. The first term in (4.16), is a single pole, so 


$$
c_{3: 2,2 ; 1}^{(2)}=-\frac{2\langle 13\rangle^{2}\left[\begin{array}{ll}
1 & 2
\end{array}\right]}{\langle 34\rangle} \frac{[a b]^{2}}{\left[b\left|P_{56} Q\right| b\right]}\left(\frac{g\left(\widetilde{\ell}_{7}\right) \widetilde{R}_{2}\left[\ell_{7}, \widetilde{\eta}, P_{56}\right]\left\langle 5|Q| \ell_{7}\right]^{2}}{\left\langle 6|Q| \ell_{7}\right]\left[\ell_{7} \widetilde{\eta}\right]\left[\ell_{7} \ell_{8}\right]}+\frac{g\left(\widetilde{\ell}_{8}\right) \widetilde{R}_{2}\left[\ell_{8}, \widetilde{\eta}, P_{56}\right]\left\langle 5|Q| \ell_{8}\right]^{2}}{\left\langle 6|Q| \ell_{8}\right]\left[\ell_{8} \widetilde{\eta}\right]\left[\ell_{8} \ell_{7}\right]}\right)
$$

where $\widetilde{R}_{2}\left[\ell_{7}, \widetilde{\eta}, P_{56}\right]$ is the conjugated version of $R_{2}$ given in Appendix B, i.e., $\left.|\bullet\rangle \leftrightarrow \mid \bullet\right]$.

The third term in (4.16) has a double pole, so the contribution is

$$
\begin{aligned}
c_{3: 2,2 ; 1}^{(3)}= & \frac{4 P_{56}^{2}\langle 13\rangle}{\langle 34\rangle} \frac{[a b]^{4}}{\left[b\left|P_{56} Q\right| b\right]^{2}} \frac{g\left(\widetilde{\ell}_{7}\right)\left[\ell_{7} 2\right]\left\langle 3|Q| \ell_{7}\right]\left\langle 5|Q| \ell_{7}\right]^{2}}{\left[\ell_{7} \ell_{8}\right]^{2}\left\langle 6|Q| \ell_{7}\right]\left[\ell_{7} \widetilde{\eta}\right]} \widetilde{R}_{2}\left[\ell_{7}, \widetilde{\eta}, P_{56}\right] \sum_{i=1}^{8} \frac{\left[L_{7, i} L_{8, i}\right]}{\left[\ell_{7} L_{7, i}\right]\left[\ell_{7} L_{8, i}\right]} \\
& \left.\left.\left.+\frac{4 P_{56}^{2}\langle 13\rangle}{\langle 34\rangle} \frac{[a b]^{4}}{\left[b\left|P_{56} Q\right| b\right]^{2}} \frac{g\left(\widetilde{\ell}_{7}\right)\left[\ell_{7} 2\right]\left\langle 3|Q| \ell_{7}\right]\left\langle 5|Q| \ell_{7}\right]^{2}}{\left[\ell_{7} \ell_{8}\right]^{2}\left\langle 6|Q| \ell_{7}\right]\left[\ell_{7} \widetilde{\eta}\right]\left[\ell_{7} 2\right]} \widetilde{R}_{3}\left[\ell_{7}, \widetilde{\eta}, \mid 2\right], P_{56}\right]+\left\{\mid \eta_{1}\right] \leftrightarrow \mid \eta_{2}\right]\right\}
\end{aligned}
$$

with the following two lists:

$$
\begin{aligned}
& \left.\left.\left.\left.\left.\left.\left.\left.\left.L_{7}=\{\mid 6], \mid 2\right], \mid 2\right],\left|P_{56}\right| 3\right\rangle,\left|P_{56}\right| 3\right\rangle,|Q| 5\right\rangle,|Q| 5\right\rangle,|Q| 3\right\rangle, \mid 2\right]\right\} \\
& \left.\left.\left.\left.\left.\left.\left.\left.\left.L_{8}=\{\mid 1],\left|P_{12}\right| 4\right\rangle,\left|P_{56}\right| 4\right\rangle,\left|P_{56} P_{34}\right| 2\right],|Q| 6\right\rangle, \mid \ell_{8}\right], \mid \ell_{8}\right], \mid \widetilde{\eta}\right],|P| \ell_{7}\right\rangle\right\}
\end{aligned}
$$

The second term in (4.16) has a triple pole, so the contribution is

$$
\begin{aligned}
c_{3: 2,2 ; 1}^{(4)} & =\frac{2\left(P_{56}^{2}\right)^{2}}{[1 \quad 2]\langle 34\rangle} \frac{[a b]^{6}}{\left[b\left|P_{56} Q\right| b\right]^{3}} \frac{g\left(\widetilde{\ell}_{7}\right)\left[\ell_{7} 2\right]^{2}\left\langle 3|Q| \ell_{7}\right]^{2}\left\langle 5|Q| \ell_{7}\right]^{2}}{\left[\ell_{7} \ell_{8}\right]^{3}\left\langle 6|Q| \ell_{7}\right]\left[\ell_{7} \widetilde{\eta}\right]} \\
& \left(\sum_{1 \leq i \leq j \leq 9} \frac{\left[L_{9, i} L_{10, i}\right]}{\left[\ell_{7} L_{9, i}\right]\left[\ell_{7} L_{10, i}\right]} \frac{\left[L_{9, j} L_{10, j}\right]}{\left[\ell_{7} L_{9, j}\right]\left[\ell_{7} L_{10, j}\right]} \widetilde{R}_{2}\left[\ell_{7}, \widetilde{\eta}, P_{56}\right]\right. \\
& +\sum_{i=1}^{9} \frac{\left[L_{9, i} L_{10, i}\right]}{\left[\ell_{7} L_{9, i}\right]\left[\ell_{7} L_{10, i}\right]} \frac{\left[L_{9,11} L_{9, i}\right]}{\left[\ell_{7} L_{9,11}\right]\left[\ell_{7} L_{9, i}\right]} \widetilde{R}_{2}\left[\ell_{7}, \widetilde{\eta}, P_{56}\right] \\
& \left.+\frac{\left[L_{9,11} L_{9, i}\right]}{\left[\ell_{7} L_{9,11}\right]\left[\ell_{7} L_{9, i}\right]\left[\ell_{7} 2\right]} \widetilde{R}_{3}\left[\ell_{7}, \widetilde{\eta}, \mid 2\right], P_{56}\right] \\
& \left.\left.\left.+\sum_{1 \leq i \leq 9} \frac{\left[L_{9, i} L_{10, i}\right]}{\left[\ell_{7} L_{9, i}\right]\left[\ell_{7} L_{10, i}\right]\left[\ell_{7} 2\right]} \widetilde{R}_{3}\left[\ell_{7}, \widetilde{\eta}, \mid 2\right], P_{56}\right]+\frac{1}{\left[\ell_{7} 2\right]^{2}} \widetilde{R}_{4}\left[\ell_{7}, \widetilde{\eta}, \mid 2\right], P_{56}\right]\right) \\
& \left.\left.+\left\{\mid \eta_{1}\right] \leftrightarrow \mid \eta_{2}\right]\right\}
\end{aligned}
$$

with the following two lists:

$$
\begin{aligned}
L_{9} & \left.\left.\left.\left.\left.\left.\left.\left.\left.=\{\mid 6], \mid 2], \mid 2],\left|P_{56}\right| 3\right\rangle,\left|P_{56}\right| 3\right\rangle,|Q| 3\right\rangle,|Q| 3\right\rangle,|Q| 5\right\rangle,|Q| 5\right\rangle, \mid 2\right], \mid 2\right]\right\} \\
L_{10} & \left.\left.\left.\left.\left.\left.\left.\left.\left.\left.=\{\mid 1],\left|P_{12}\right| 4\right\rangle,\left|P_{56}\right| 4\right\rangle,\left|P_{56} P_{34}\right| 2\right],|Q| 6\right\rangle, \mid \ell_{8}\right], \mid \ell_{8}\right], \mid 8\right], \mid \widetilde{\eta}\right],|P| \ell_{7}\right\rangle\right\} .
\end{aligned}
$$


The result of cut $C_{56}$

The coefficient of bubble $I_{2: 2 ; 5}$ is the sum of (4.9), (4.11), (4.23), (4.24), (4.25), (4.27), and (4.28):

$$
c_{2: 2 ; 5}=c_{2: 2 ; 5}^{(3 r)}+c_{2: 2 ; 5}^{1 r ; A 1}+c_{2: 2 ; 5}^{1 r ; A 2-1}+c_{2: 2 ; 5}^{1 r ; A 2-2}+c_{2: 2 ; 5}^{1 r ; A 3-1}+c_{2: 2 ; 5}^{1 r ; A 3-2} .
$$

The coefficient of the three-mass triangle function $I_{3: 2,2 ; 1}$ is given by the sum of (4.30), (4.32), (4.33), and (4.35):

$$
c_{3: 2,2 ; 1}=c_{3: 2,2 ; 1}^{(1)}+c_{3: 2,2 ; 1}^{(2)}+c_{3: 2,2 ; 1}^{(3)}+c_{3: 2,2 ; 1}^{(4)} .
$$

The result could have been written directly using the functions defined at the end of Appendix C. Here we have given some intermediate steps for illustration. For further details, see Appendix C.

5. $A\left(1^{-}, 2^{-}, 3^{+}, 4^{-}, 5^{+}, 6^{+}\right)$

The last of the NMHV six-gluon helicity configurations requires the heaviest computation. However, there are no essentially new features encountered. In this section we present, with minimal discussion, some of our intermediate steps in order to allow the reader to confirm our final formulas for the coefficients.

This helicity configuration is invariant under a $\mathbb{Z}_{2}$ symmetry generated by $\alpha: i \leftrightarrow 7-i$ accompanied by conjugation. There are box, triangle and bubble contributions. The box coefficients are straightforward to calculate by quadruple cuts and have been given in [42]. We list them again here in the notation consistent with the rest of this paper.10

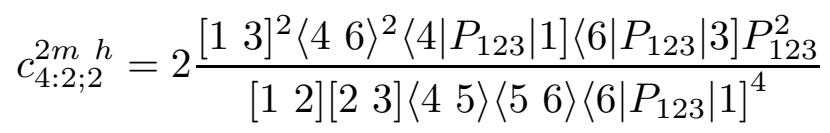

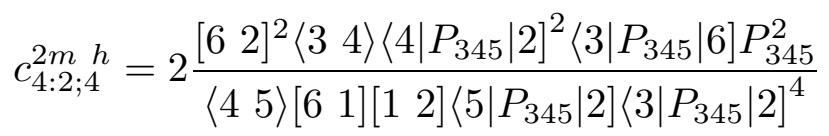

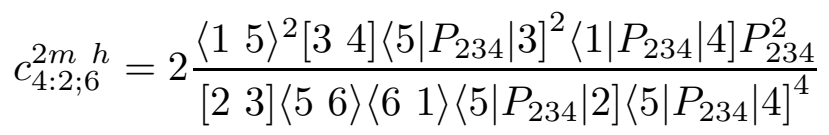

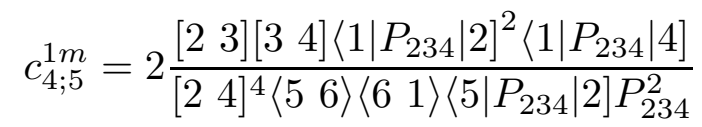

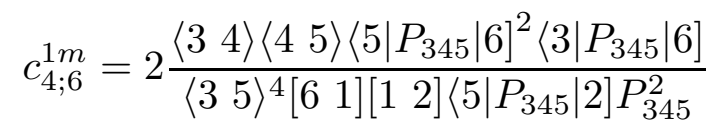

10 An apparent discrepancy is due to a typo in the numerator of the coefficient $c_{5}^{\mathcal{N}}=0$ in 42 . 
In this configuration, there is only one nonvanishing three-mass-triangle coefficient, with the distribution $(23|45| 61)$.

For the bubble part, we have the following cuts: three particle channels $C_{123}, C_{612}$ and $C_{234}$; two particle channels $C_{23}, C_{34}, C_{45}$ and $C_{61}$. Among these, the pairs $\left(C_{612}, C_{234}\right)$ and $\left(C_{23}, C_{45}\right)$ are related by the $Z_{2}$ symmetry, while the others are invariant. So in total we have five independent double cuts $C_{123}, C_{234}, C_{23}, C_{34}$ and $C_{61}$. We address these one by one.

Throughout this section we freely omit the integral sign when its presence may be inferred from spinor differentials.

\subsection{Cut $C_{123}$}

For $C_{123}$, there is no three-mass triangle contribution and the calculation will be relatively simple. The expression is given by

$$
\begin{aligned}
C_{123}=\int d \mu & {\left[A\left(\ell_{1}^{+}, 1^{-}, 2^{-}, 3^{+}, \ell_{2}^{-}\right) A\left(\ell_{2}^{+}, 4^{-}, 5^{+}, 6^{+}, \ell_{1}^{-}\right)\right.} \\
& \left.+A\left(\ell_{1}^{-}, 1^{-}, 2^{-}, 3^{+}, \ell_{2}^{+}\right) A\left(\ell_{2}^{-}, 4^{-}, 5^{+}, 6^{+}, \ell_{1}^{+}\right)\right] \\
= & \int d \mu \frac{2\left\langle 4 \ell_{1}\right\rangle^{2}\left\langle 4 \ell_{2}\right\rangle^{2}\left[3 \ell_{1}\right]^{2}\left[3 \ell_{2}\right]}{\langle 45\rangle\langle 56\rangle\left\langle 6 \ell_{1}\right\rangle\left\langle\ell_{1} \ell_{2}\right\rangle\left\langle\ell_{2} 4\right\rangle[12][23]\left[\ell_{1} 1\right]\left[\ell_{2} \ell_{1}\right]}
\end{aligned}
$$

After the $t$-integration, the rational contribution can be read as a sum of three contributions,

$$
C_{123}^{r a t}=C_{123}^{(1 r)}+C_{123}^{(2 r)}+C_{123}^{(3 r)}
$$

each of which will be discussed separately.

The term $C_{123}^{(1 r)}$

$$
C_{123}^{(1 r)}=\frac{2\langle\ell d \ell\rangle[\ell d \ell]\langle 4 \ell\rangle^{2}\left\langle 4\left|P_{123}\right| 1\right]\left\langle\ell\left|P_{123}\right| 3\right]^{2}[31]\left(P_{123}^{2}\right)}{\langle 45\rangle\langle 56\rangle\langle 6 \ell\rangle\left\langle\ell\left|P_{123}\right| 1\right]^{3}\left\langle\ell\left|P_{123}\right| \ell\right]^{2}[12][23]} .
$$

This integrand may be turned into a full derivative by choosing as a reference spinor $\mid \eta]=P_{123}|1\rangle$, to neutralize the multiple pole $\left\langle\ell\left|P_{123}\right| 1\right]^{3}$ :

$$
C_{123}^{(1 r)}=\frac{2\langle\ell d \ell\rangle\left[d \ell \partial_{\ell}\right]\langle 4 \ell\rangle^{2}\left\langle 1\left|P_{123}\right| \ell\right]\left\langle 4\left|P_{123}\right| 1\right]\left\langle\ell\left|P_{123}\right| 3\right]^{2}[31]}{\langle 1 \ell\rangle\langle 45\rangle\langle 56\rangle\langle 6 \ell\rangle\left\langle\ell\left|P_{123}\right| 1\right]^{3}\left\langle\ell\left|P_{123}\right| \ell\right][12][23]} .
$$

The sum of the residues can therefore be performed as follows:

$$
C_{123}^{(1 r: s)}=\frac{2\left\langle 4\left|P_{123}\right| 1\right][31]}{\langle 45\rangle\langle 56\rangle[12][23]} \sum_{i=1,2} \lim _{\ell \rightarrow \ell_{i}}\left\langle\ell \ell_{i}\right\rangle \frac{\langle 4 \ell\rangle^{2}\left\langle 1\left|P_{123}\right| \ell\right]\left\langle\ell\left|P_{123}\right| 3\right]^{2}}{\langle 1 \ell\rangle\langle 6 \ell\rangle\left\langle\ell\left|P_{123}\right| 1\right]^{3}\left\langle\ell\left|P_{123}\right| \ell\right]},
$$


with $\left|\ell_{1}\right\rangle=|1\rangle$ and $\left|\ell_{2}\right\rangle=|6\rangle$.

The term $C_{123}^{(2 r)}$

$$
C_{123}^{(2 r)}=\frac{2\langle\ell d \ell\rangle[\ell d \ell]\langle 4 \ell\rangle^{2}\left\langle 4\left|P_{123}\right| 1\right]\left\langle\ell\left|P_{123}\right| 3\right]^{2}[3 \ell]\left(P_{123}^{2}\right)}{\langle 45\rangle\langle 56\rangle\langle 6 \ell\rangle\left\langle\ell\left|P_{123}\right| 1\right]^{2}\left\langle\ell\left|P_{123}\right| \ell\right]^{3}[12][23]} .
$$

In this case, one can write the integrand as a full derivative by choosing the reference spinor $\eta=3$, so that

$$
C_{123}^{(2 r)}=-\frac{\langle\ell d \ell\rangle\left[d \ell \partial_{\ell}\right]\langle 4 \ell\rangle^{2}\left\langle 4\left|P_{123}\right| 1\right]\left\langle\ell\left|P_{123}\right| 3\right][3 \ell]^{2}\left(P_{123}^{2}\right)}{\langle 45\rangle\langle 56\rangle\langle 6 \ell\rangle\left\langle\ell\left|P_{123}\right| 1\right]^{2}\left\langle\ell\left|P_{123}\right| \ell\right]^{2}[12][23]} .
$$

Note the presence of a double pole, $\left\langle\ell\left|P_{123}\right| 1\right]^{2}$.

The residue of the single pole $\ell=6$ is

$$
C_{123}^{(2 r: s)}=-\frac{\langle 46\rangle^{2}\left\langle 4\left|P_{123}\right| 1\right]\left\langle 6\left|P_{123}\right| 3\right][36]^{2}\left(P_{123}^{2}\right)}{\langle 45\rangle\langle 56\rangle\left\langle 6\left|P_{123}\right| 1\right]^{2}\left\langle 6\left|P_{123}\right| 6\right]^{2}[12][23]},
$$

while the residue of the double pole $\left.|\ell\rangle=P_{123} \mid 1\right]$ is

$$
\left.C_{123}^{(2 r: d)}=-\frac{\left\langle 4\left|P_{123}\right| 1\right]\left[3\left|P_{123}\right| 1\right\rangle^{2}}{\langle 45\rangle\langle 56\rangle[12][23]\left(P_{123}^{2}\right)} P_{2}\left[P_{123} \mid 1\right], L_{1}^{I I: C_{123}}, L_{2}^{I I: C_{123}}\right]
$$

with

$$
\begin{aligned}
& \left.L_{1}^{I I: C_{123}}=\left\{|4\rangle,|4\rangle, P_{123} \mid 3\right]\right\} \\
& L_{2}^{I I: C_{123}}=\{|6\rangle,|1\rangle,|1\rangle\}
\end{aligned}
$$

since, having chosen $\mid \ell]=P_{123}|1\rangle$, we used $\left\langle\ell\left|P_{123}\right| \ell\right] \rightarrow P_{123}^{2}\langle\ell 1\rangle$.

The term $C_{123}^{(3 r)}$

$$
C_{123}^{(3 r)}=\frac{2\langle\ell d \ell\rangle[\ell d \ell]\langle 4 \ell\rangle^{3}\left\langle\ell\left|P_{123}\right| 3\right][3 \ell]^{2}\left(P_{123}^{2}\right)^{2}}{\langle 45\rangle\langle 56\rangle\langle 6 \ell\rangle\left\langle\ell\left|P_{123}\right| 1\right]\left\langle\ell\left|P_{123}\right| \ell\right]^{4}[12][23]} .
$$

It is straightforward to write it as a full derivative with $\eta=3$ :

$$
C_{123}^{(3 r)}=-\frac{2\langle\ell d \ell\rangle\left[d \ell \partial_{\ell}\right]\langle 4 \ell\rangle^{3}[3 \ell]^{3}\left(P_{123}^{2}\right)^{2}}{3\langle 45\rangle\langle 56\rangle\langle 6 \ell\rangle\left\langle\ell\left|P_{123}\right| 1\right]\left\langle\ell\left|P_{123}\right| \ell\right]^{3}[12][23]} .
$$

We obtain an expression where only single poles are present, whose sum of residues is

$$
C_{123}^{(3 r: s)}=-\frac{2\left(P_{123}^{2}\right)^{2}}{3\langle 45\rangle\langle 56\rangle[12][23]} \sum_{i=2,3} \lim _{\ell \rightarrow \ell_{i}}\left\langle\ell \ell_{i}\right\rangle \frac{\langle 4 \ell\rangle^{3}[3 \ell]^{3}}{\langle 6 \ell\rangle\left\langle\ell\left|P_{123}\right| 1\right]\left\langle\ell\left|P_{123}\right| \ell\right]^{3}},
$$

with $\left|\ell_{2}\right\rangle=|6\rangle$ and $\left.\left|\ell_{3}\right\rangle=P_{123} \mid 1\right]$

Finally, the coefficient of the bubble $I_{2: 3 ; 1}$ is obtained by adding (5.6), (5.9), (5.10), and (5.14):

$$
c_{2: 3 ; 1}=C_{123}^{(1 r: s)}+C_{123}^{(2 r: s)}+C_{123}^{(2 r: d)}+C_{123}^{(3 r: s)}
$$




\subsection{Cut $C_{234}$}

The cut $C_{234}$ is given by

$$
\begin{aligned}
C_{234}=\int d \mu & {\left[A\left(\ell_{1}^{+}, 2^{-}, 3^{+}, 4^{-}, \ell_{2}^{-}\right) A\left(\ell_{2}^{+}, 5^{+}, 6^{+}, 1^{-}, \ell_{1}^{-}\right)\right.} \\
& \left.+A\left(\ell_{1}^{-}, 2^{-}, 3^{+}, 4^{-}, \ell_{2}^{+}\right) A\left(\ell_{2}^{-}, 5^{+}, 6^{+}, 1^{-}, \ell_{1}^{+}\right)\right] \\
= & \int d \mu \frac{2\left\langle 1 \ell_{1}\right\rangle\left\langle 1 \ell_{2}\right\rangle^{2}\left[3 \ell_{1}\right]^{2}\left[3 \ell_{2}\right]^{2}}{\langle 56\rangle\langle 61\rangle\left\langle\ell_{1} \ell_{2}\right\rangle\left\langle\ell_{2} 5\right\rangle[23][34]\left[4 \ell_{2}\right]\left[\ell_{1} 2\right]\left[\ell_{2} \ell_{1}\right]}
\end{aligned}
$$

After the $t$-integration, the rational contribution can be read as a sum of three contributions,

$$
C_{234}^{r a t}=C_{234}^{(1 r)}+C_{234}^{(2 r)}+C_{234}^{(3 r)}
$$

each of which will be discussed separately.

The term $C_{234}^{(1 r)}$

$$
C_{234}^{(1 r)}=-\frac{2\langle\ell d \ell\rangle[\ell d \ell]\langle 1 \ell\rangle^{2}\left\langle 1\left|P_{234}\right| 4\right]\left\langle\ell\left|P_{234}\right| 3\right]^{3}\left(P_{234}^{2}\right)}{\langle 56\rangle\langle 5 \ell\rangle\langle 61\rangle\left\langle\ell\left|P_{234}\right| 2\right]\left\langle\ell\left|P_{234}\right| 4\right]^{3}\left\langle\ell\left|P_{234}\right| \ell\right]^{2}[23]} .
$$

We write it as a full derivative by choosing the reference spinor $\mid \eta]=P_{234}|4\rangle$ in order to neutralize the multiple pole $\left\langle\ell\left|P_{23}\right| 4\right]^{3}$ :

$$
C_{234}^{(1 r)}=-\frac{2\langle\ell d \ell\rangle\left[d \ell \partial_{\ell}\right]\langle 1 \ell\rangle^{2}\left\langle 1\left|P_{234}\right| 4\right]\left\langle 4\left|P_{234}\right| \ell\right]\left\langle\ell\left|P_{234}\right| 3\right]^{3}}{\langle 4 \ell\rangle\langle 56\rangle\langle 5 \ell\rangle\langle 61\rangle\left\langle\ell\left|P_{234}\right| 2\right]\left\langle\ell\left|P_{234}\right| 4\right]^{3}\left\langle\ell\left|P_{234}\right| \ell\right][23]}
$$

The sum of the residues can be therefore performed as follows:

$$
C_{234}^{(1 r: s)}=-\frac{2\left\langle 1\left|P_{234}\right| 4\right]}{\langle 56\rangle\langle 61\rangle[23]} \sum_{i=1,2,3} \lim _{\ell \rightarrow \ell_{i}}\left\langle\ell \ell_{i}\right\rangle \frac{\langle 1 \ell\rangle^{2}\left\langle 4\left|P_{234}\right| \ell\right]\left\langle\ell\left|P_{234}\right| 3\right]^{3}}{\langle 4 \ell\rangle\langle 5 \ell\rangle\left\langle\ell\left|P_{234}\right| 2\right]\left\langle\ell\left|P_{234}\right| 4\right]^{3}\left\langle\ell\left|P_{234}\right| \ell\right]},
$$

with $\left|\ell_{1}\right\rangle=|4\rangle,\left|\ell_{2}\right\rangle=|5\rangle$, and $\left.\left|\ell_{3}\right\rangle=P_{234} \mid 2\right]$.

The term $C_{234}^{(2 r)}$

$$
C_{234}^{(2 r)}=-\frac{2\langle\ell d \ell\rangle[\ell d \ell]\langle 1 \ell\rangle^{2}\left\langle 1\left|P_{234}\right| 4\right]\left\langle\ell\left|P_{234}\right| 3\right]^{3}[3 \ell]\left(P_{234}^{2}\right)}{\langle 56\rangle\langle 5 \ell\rangle\langle 61\rangle\left\langle\ell\left|P_{234}\right| 2\right]\left\langle\ell\left|P_{234}\right| 4\right]^{2}\left\langle\ell\left|P_{234}\right| \ell\right]^{3}[23][34]}
$$

We write it as a full derivative by choosing the reference spinor $\eta=3$ :

$$
C_{234}^{(2 r)}=\frac{\langle\ell d \ell\rangle\left[d \ell \partial_{\ell}\right]\langle 1 \ell\rangle^{2}\left\langle 1\left|P_{234}\right| 4\right]\left\langle\ell\left|P_{234}\right| 3\right]^{2}[3 \ell]^{2}\left(P_{234}^{2}\right)}{\langle 56\rangle\langle 5 \ell\rangle\langle 61\rangle\left\langle\ell\left|P_{234}\right| 2\right]\left\langle\ell\left|P_{234}\right| 4\right]^{2}\left\langle\ell\left|P_{234}\right| \ell\right]^{2}[23][34]} .
$$


The sum of the residues of the single pole is

$$
C_{234}^{(2 r: s)}=\frac{\left\langle 1\left|P_{234}\right| 4\right]\left(P_{234}^{2}\right)}{\langle 56\rangle\langle 61\rangle[23][34]} \sum_{i=2,3} \lim _{\ell \rightarrow \ell_{i}}\left\langle\ell \ell_{i}\right\rangle \frac{\langle 1 \ell\rangle^{2}\left\langle\ell\left|P_{234}\right| 3\right]^{2}[3 \ell]^{2}}{\langle 5 \ell\rangle\left\langle\ell\left|P_{234}\right| 2\right]\left\langle\ell\left|P_{234}\right| 4\right]^{2}\left\langle\ell\left|P_{234}\right| \ell\right]^{2}},
$$

with $\left|\ell_{2}\right\rangle=|5\rangle$, and $\left.\left|\ell_{3}\right\rangle=P_{234} \mid 2\right]$. The residue of double pole $\left.|\ell\rangle=P_{234} \mid 4\right]$ can be written as

$$
\left.C_{234}^{(2 r: d)}=\frac{\left\langle 1\left|P_{234}\right| 4\right]\left\langle 4\left|P_{234}\right| 3\right]^{2}}{\left(P_{234}^{2}\right)\langle 56\rangle\langle 61\rangle[23][34]} P_{2}\left[P_{234} \mid 4\right], L_{1}^{I I: C_{234}}, L_{2}^{I I: C_{234}}\right],
$$

with

$$
\begin{aligned}
& \left.\left.L_{1}^{I I: C_{234}}=\left\{|1\rangle,|1\rangle, P_{234} \mid 3\right], P_{234} \mid 3\right]\right\} \\
& \left.L_{2}^{I I: C_{234}}=\left\{|5\rangle, P_{234} \mid 2\right],|4\rangle,|4\rangle\right\}
\end{aligned}
$$

since, having chosen $\mid \ell]=P_{234}|4\rangle$, we used $\left\langle\ell\left|P_{234}\right| \ell\right] \rightarrow P_{234}^{2}\langle\ell 4\rangle$.

The term $C_{234}^{(3 r)}$

$$
C_{234}^{(3 r)}=-\frac{2\langle\ell d \ell\rangle[\ell d \ell]\langle 1 \ell\rangle^{3}\left\langle\ell\left|P_{234}\right| 3\right]^{2}[3 \ell]^{2}\left(P_{234}^{2}\right)^{2}}{\langle 56\rangle\langle 5 \ell\rangle\langle 61\rangle\left\langle\ell\left|P_{234}\right| 2\right]\left\langle\ell\left|P_{234}\right| 4\right]\left\langle\ell\left|P_{234}\right| \ell\right]^{4}[23][34]} .
$$

In this case only simple poles are present. We can write it as a full derivative with $\eta=3$ :

$$
C_{234}^{(3 r)}=\frac{2\langle\ell d \ell\rangle\left[d \ell \partial_{\ell}\right]\langle 1 \ell\rangle^{3}\left\langle\ell\left|P_{234}\right| 3\right][3 \ell]^{3}\left(P_{234}^{2}\right)^{2}}{3\langle 56\rangle\langle 5 \ell\rangle\langle 61\rangle\left\langle\ell\left|P_{234}\right| 2\right]\left\langle\ell\left|P_{234}\right| 4\right]\left\langle\ell\left|P_{234}\right| \ell\right]^{3}[23][34]} .
$$

We obtain an expression where only single poles are present, whose sum of residues is

$$
C_{123}^{(3 r: s)}=\frac{2\left(P_{234}^{2}\right)^{2}}{3\langle 56\rangle\langle 61\rangle[23][34]} \sum_{i=2,3,4} \lim _{\ell \rightarrow \ell_{i}}\left\langle\ell \ell_{i}\right\rangle \frac{\langle 1 \ell\rangle^{3}\left\langle\ell\left|P_{234}\right| 3\right][3 \ell]^{3}}{\langle 5 \ell\rangle\left\langle\ell\left|P_{234}\right| 2\right]\left\langle\ell\left|P_{234}\right| 4\right]\left\langle\ell\left|P_{234}\right| \ell\right]^{3}}
$$

with $\left.\left|\ell_{2}\right\rangle=|5\rangle,\left|\ell_{3}\right\rangle=P_{234} \mid 2\right]$ and $\left.\left|\ell_{4}\right\rangle=P_{234} \mid 4\right]$.

Finally the coefficient of the bubble $I_{2: 3 ; 2}$ is obtained by adding (5.20), (5.23), (5.24), and $(5.28)$ :

$$
c_{2: 3 ; 2}=C_{234}^{(1 r: s)}+C_{234}^{(2 r: s)}+C_{234}^{(3 r: d)}+C_{234}^{(3 r: s)}
$$




\section{3. $C u t C_{34}$}

For this double cut, there is no triangle contribution and the result is simpler. The cut is given by

$$
\begin{aligned}
C_{34}=\int d \mu & {\left[A\left(\ell_{1}^{+}, 3^{+}, 4^{-}, \ell_{2}^{-}\right) A\left(\ell_{2}^{+}, 5^{+}, 6^{+}, 1^{-}, 2^{-}, \ell_{1}^{-}\right)\right.} \\
& \left.+A\left(\ell_{1}^{-}, 3^{+}, 4^{-}, \ell_{2}^{+}\right) A\left(\ell_{2}^{-}, 5^{+}, 6^{+}, 1^{-}, 2^{-}, \ell_{1}^{+}\right)\right] \\
=\int d \mu & \left\{\frac{2\left\langle 4 \ell_{1}\right\rangle^{2}\left\langle 4 \ell_{2}\right\rangle\left\langle 1\left|P_{56}\right| \ell_{1}\right]^{2}\left\langle 1\left|P_{56}\right| \ell_{2}\right]}{\langle 34\rangle\langle 56\rangle\langle 61\rangle\left\langle\ell_{1} 3\right\rangle\left\langle\ell_{2} \ell_{1}\right\rangle\left\langle 5\left|P_{61}\right| 2\right]\left[2 \ell_{1}\right]\left[\ell_{1} \ell_{2}\right]\left(P_{561}^{2}\right)}\right. \\
& \left.+\frac{2\left\langle 4 \ell_{1}\right\rangle^{2}\left\langle 4 \ell_{2}\right\rangle\left\langle\ell_{1}\left|P_{12}\right| 6\right]\left\langle\ell_{2}\left|P_{12}\right| 6\right]^{2}}{\langle 34\rangle\left\langle\ell_{1} 3\right\rangle\left\langle\ell_{1} \ell_{2}\right\rangle\left\langle\ell_{2} 5\right\rangle\left\langle\ell_{2} \ell_{1}\right\rangle\left\langle 5\left|P_{61}\right| 2\right][12][61]\left(P_{345}^{2}\right)}\right\} \\
=C_{34}^{(1)}+ & C_{34}^{(2)}
\end{aligned}
$$

Due to the possibility of writing $A\left(\ell_{1}^{+}, 3^{+}, 4^{-}, \ell_{2}^{-}\right)$in terms of either holomorphic or antiholomorphic spinor products, as follows,

$$
A\left(\ell_{1}^{+}, 3^{+}, 4^{-}, \ell_{2}^{-}\right)=\frac{\left\langle 4 \ell_{1}\right\rangle^{2}\left\langle 4 \ell_{2}\right\rangle^{2}}{\left\langle\ell_{1} 3\right\rangle\langle 34\rangle\left\langle 4 \ell_{2}\right\rangle\left\langle\ell_{2} \ell_{1}\right\rangle}=\frac{\left[3 \ell_{1}\right]^{2}\left[3 \ell_{2}\right]^{2}}{\left[\ell_{1} 3\right][34]\left[4 \ell_{2}\right]\left[\ell_{2} \ell_{1}\right]},
$$

one can rewrite $C_{34}^{(2)}$ as

$$
\begin{aligned}
C_{34}^{(2)} & =\frac{2\left\langle 4 \ell_{1}\right\rangle^{2}\left\langle 4 \ell_{2}\right\rangle\left\langle\ell_{1}\left|P_{12}\right| 6\right]\left\langle\ell_{2}\left|P_{12}\right| 6\right]^{2}}{\langle 34\rangle\left\langle\ell_{1} 3\right\rangle\left\langle\ell_{1} \ell_{2}\right\rangle\left\langle\ell_{2} 5\right\rangle\left\langle\ell_{2} \ell_{1}\right\rangle\left\langle 5\left|P_{61}\right| 2\right][12][61]\left(P_{345}^{2}\right)} \\
& =\frac{2\left[3 \ell_{2}\right]^{2}\left[3 \ell_{1}\right]\left\langle\ell_{1}\left|P_{12}\right| 6\right]\left\langle\ell_{2}\left|P_{12}\right| 6\right]^{2}}{[34]\left[\ell_{2} 4\right]\left[\ell_{2} \ell_{1}\right]\left\langle\ell_{2} 5\right\rangle\left\langle\ell_{2} \ell_{1}\right\rangle\left\langle 5\left|P_{61}\right| 2\right][12][61]\left(P_{345}^{2}\right)} .
\end{aligned}
$$

In this shape, $C_{34}^{(2)}$ can be obtained from $C_{34}^{(1)}$, as the following relation holds,

$$
P_{345}^{2} C_{34}(2,0)=(-1) \operatorname{Tfm}\left\{P_{561}^{2} C_{34}(1,0)\right\}
$$

where Tfm is the composition of three operations: i) Parity: $\langle,\rangle \leftrightarrow[$, ]; ii) relabeling: $\{1,2,3,4,5,6\} \rightarrow\{6,5,4,3,2,1\} ;$ iii) exchange: $\ell_{1} \leftrightarrow \ell_{2}$. Therefore one can worry only about $C_{34}^{(1)}$ and recover $C_{34}^{(2)}$ at the end through the above relation. 
After the $t$-integration, the rational contribution coming from $C_{34}^{(1)}$ can be read as

$$
\begin{aligned}
C_{34}^{(1 r)} & =\frac{2\langle\ell d \ell\rangle[\ell d \ell]\left\langle 1\left|P_{56}\right| \ell\right]^{3}[3 \ell][43] P_{34}}{\langle 56\rangle\langle 61\rangle\left\langle 5\left|P_{61}\right| 2\right]\left\langle\ell\left|P_{34}\right| \ell\right]^{2}[2 \ell][4 \ell]^{3}\left(P_{561}^{2}\right)} \\
& -\frac{2\langle\ell d \ell\rangle[\ell d \ell]\left\langle 1\left|P_{56}\right| 3\right]\left\langle 1\left|P_{56}\right| \ell\right]^{2}[3 \ell] P_{34}}{\langle 56\rangle\langle 61\rangle\left\langle 5\left|P_{61}\right| 2\right]\left\langle\ell\left|P_{34}\right| \ell\right]^{2}[2 \ell][4 \ell]^{2}\left(P_{561}^{2}\right)} \\
& -\frac{2\langle\ell d \ell\rangle[\ell d \ell]\langle 4 \ell\rangle\left\langle 1\left|P_{56}\right| \ell\right]^{3}[3 \ell][43] P_{34}}{\langle 56\rangle\langle 61\rangle\left\langle 5\left|P_{61}\right| 2\right]\left\langle\ell\left|P_{34}\right| \ell\right]^{3}[2 \ell][4 \ell]^{2}\left(P_{561}^{2}\right)} \\
& +\frac{2\langle\ell d \ell\rangle[\ell d \ell]\langle 4 \ell\rangle\left\langle 1\left|P_{56}\right| 3\right]\left\langle 1\left|P_{56}\right| \ell\right]^{2}[3 \ell] P_{34}}{\langle 56\rangle\langle 61\rangle\left\langle 5\left|P_{61}\right| 2\right]\left\langle\ell\left|P_{34}\right| \ell\right]^{3}[2 \ell][4 \ell]\left(P_{561}^{2}\right)} \\
& +\frac{2\langle\ell d \ell\rangle[\ell d \ell]\langle 4 \ell\rangle^{2}\left\langle 1\left|P_{56}\right| \ell\right]^{3}[3 \ell][43] P_{34}}{\langle 56\rangle\langle 61\rangle\left\langle 5\left|P_{61}\right| 2\right]\left\langle\ell\left|P_{34}\right| \ell\right]^{4}[2 \ell][4 \ell]\left(P_{561}^{2}\right)} .
\end{aligned}
$$

This can be written as a full derivative by choosing as reference spinor $\eta=4$ :

$$
\begin{aligned}
C_{34}^{(1 r)} & =\frac{2[\ell d \ell]\left\langle d \ell \partial_{\ell}\right\rangle\langle 4 \ell\rangle\left\langle 1\left|P_{56}\right| \ell\right]^{3}[43] P_{34}}{\langle 43\rangle\langle 56\rangle\langle 61\rangle\left\langle 5\left|P_{61}\right| 2\right]\left\langle\ell\left|P_{34}\right| \ell\right][2 \ell][4 \ell]^{3}\left(P_{561}^{2}\right)} \\
& -\frac{2[\ell d \ell]\left\langle d \ell \partial_{\ell}\right\rangle\langle 4 \ell\rangle\left\langle 1\left|P_{56}\right| 3\right]\left\langle 1\left|P_{56}\right| \ell\right]^{2} P_{34}}{\langle 43\rangle\langle 56\rangle\langle 61\rangle\left\langle 5\left|P_{61}\right| 2\right]\left\langle\ell\left|P_{34}\right| \ell\right][2 \ell][4 \ell]^{2}\left(P_{561}^{2}\right)} \\
& -\frac{[\ell d \ell]\left\langle d \ell \partial_{\ell}\right\rangle\langle 4 \ell\rangle^{2}\left\langle 1\left|P_{56}\right| \ell\right]^{3}[43] P_{34}}{\langle 43\rangle\langle 56\rangle\langle 61\rangle\left\langle 5\left|P_{61}\right| 2\right]\left\langle\ell\left|P_{34}\right| \ell\right]^{2}[2 \ell][4 \ell]^{2}\left(P_{561}^{2}\right)} \\
& +\frac{[\ell d \ell]\left\langle d \ell \partial_{\ell}\right\rangle\langle 4 \ell\rangle^{2}\left\langle 1\left|P_{56}\right| 3\right]\left\langle 1\left|P_{56}\right| \ell\right]^{2} P_{34}}{\langle 43\rangle\langle 56\rangle\langle 61\rangle\left\langle 5\left|P_{61}\right| 2\right]\left\langle\ell\left|P_{34}\right| \ell\right]^{2}[2 \ell][4 \ell]\left(P_{561}^{2}\right)} \\
& +\frac{2[\ell d \ell]\left\langle d \ell \partial_{\ell}\right\rangle\langle 4 \ell\rangle^{3}\left\langle 1\left|P_{56}\right| \ell\right]^{3}[43] P_{34}}{3\langle 43\rangle\langle 56\rangle\langle 61\rangle\left\langle 5\left|P_{61}\right| 2\right]\left\langle\ell\left|P_{34}\right| \ell\right]^{3}[2 \ell][4 \ell]\left(P_{561}^{2}\right)}
\end{aligned}
$$

As one can see, the higher pole $[4 \ell]^{n}$ is neutralized, and the only active pole is $\ell=2$, whose residue reads as follows:

$$
\begin{aligned}
C_{34}^{(1 r: s)} & =-\frac{2\langle 42\rangle\left\langle 1\left|P_{56}\right| 2\right]^{2}\left\langle 1\left|P_{56}\right| 3\right] P_{34}}{\langle 43\rangle\langle 56\rangle\langle 61\rangle\left\langle 2\left|P_{34}\right| 2\right]\left\langle 5\left|P_{61}\right| 2\right][24]^{2}\left(P_{561}^{2}\right)} \\
& -\frac{\langle 42\rangle^{2}\left\langle 1\left|P_{56}\right| 2\right]^{2}\left\langle 1\left|P_{56}\right| 3\right] P_{34}}{\langle 43\rangle\langle 56\rangle\langle 61\rangle\left\langle 2\left|P_{34}\right| 2\right]^{2}\left\langle 5\left|P_{61}\right| 2\right][24]\left(P_{561}^{2}\right)} \\
& -\frac{2\langle 42\rangle\left\langle 1\left|P_{56}\right| 2\right]^{3}[43] P_{34}}{\langle 43\rangle\langle 56\rangle\langle 61\rangle\left\langle 2\left|P_{34}\right| 2\right]\left\langle 5\left|P_{61}\right| 2\right][24]^{3}\left(P_{561}^{2}\right)} \\
& -\frac{\langle 42\rangle^{2}\left\langle 1\left|P_{56}\right| 2\right]^{3}[43] P_{34}}{\langle 43\rangle\langle 56\rangle\langle 61\rangle\left\langle 2\left|P_{34}\right| 2\right]^{2}\left\langle 5\left|P_{61}\right| 2\right][24]^{2}\left(P_{561}^{2}\right)} \\
& -\frac{2\langle 42\rangle^{3}\left\langle 1\left|P_{56}\right| 2\right]^{3}[43] P_{34}}{3\langle 43\rangle\langle 56\rangle\langle 61\rangle\left\langle 2\left|P_{34}\right| 2\right]^{3}\left\langle 5\left|P_{61}\right| 2\right][24]\left(P_{561}^{2}\right)}
\end{aligned}
$$


Through the relation in eq. $(5.33)$ one can get the other term contributing to the cut,

$$
\begin{aligned}
C_{34}^{(2 r: s)} & =\frac{2\left\langle 4\left|P_{12}\right| 6\right]\left\langle 5\left|P_{12}\right| 6\right]^{2}[35] P_{34}}{\langle 53\rangle^{2}\left\langle 5\left|P_{34}\right| 5\right]\left\langle 5\left|P_{61}\right| 2\right][16][21][34]\left(P_{612}^{2}\right)} \\
& +\frac{2\langle 34\rangle\left\langle 5\left|P_{12}\right| 6\right]^{3}[35] P_{34}}{\langle 53\rangle^{3}\left\langle 5\left|P_{34}\right| 5\right]\left\langle 5\left|P_{61}\right| 2\right][16][21][34]\left(P_{612}^{2}\right)} \\
& +\frac{\left\langle 4\left|P_{12}\right| 6\right]\left\langle 5\left|P_{12}\right| 6\right]^{2}[35]^{2} P_{34}}{\langle 53\rangle\left\langle 5\left|P_{34}\right| 5\right]^{2}\left\langle 5\left|P_{61}\right| 2\right][16][21][34]\left(P_{612}^{2}\right)} \\
& +\frac{\langle 34\rangle\left\langle 5\left|P_{12}\right| 6\right]^{3}[35]^{2} P_{34}}{\langle 53\rangle^{2}\left\langle 5\left|P_{34}\right| 5\right]^{2}\left\langle 5\left|P_{61}\right| 2\right][16][21][34]\left(P_{612}^{2}\right)} \\
& +\frac{2\langle 34\rangle\left\langle 5\left|P_{12}\right| 6\right]^{3}[35]^{3} P_{34}}{3\langle 53\rangle\left\langle 5\left|P_{34}\right| 5\right]^{3}\left\langle 5\left|P_{61}\right| 2\right][16][21][34]\left(P_{612}^{2}\right)} .
\end{aligned}
$$

Finally, the coefficient of the bubble $I_{2: 2 ; 3}$ is obtained by adding (5.36) and (5.37):

$$
c_{2: 2 ; 3}=C_{34}^{(1 r: s)}+C_{34}^{(2 r: s)}
$$

\subsection{Cut $C_{23}$}

The cut in the $P_{23}$-channel receives two contributions:

$$
\begin{aligned}
C_{23}=\int d \mu & {\left[A\left(\ell_{1}^{+}, 2^{-}, 3^{+}, \ell_{2}^{-}\right) A\left(\ell_{2}^{+}, 4^{-}, 5^{+}, 6^{+}, 1^{-}, \ell_{1}^{-}\right)\right.} \\
& \left.+A\left(\ell_{1}^{-}, 2^{-}, 3^{+}, \ell_{2}^{+}\right) A\left(\ell_{2}^{-}, 4^{-}, 5^{+}, 6^{+}, 1^{-}, \ell_{1}^{+}\right)\right] \\
= & 2 C_{23}^{(1)},
\end{aligned}
$$

where

$$
\begin{aligned}
C_{23}^{(1)} & =\int d \mu\left\{\frac{\left\langle 1 \ell_{1}\right\rangle\left\langle 1 \ell_{2}\right\rangle^{2}\left\langle 2 \ell_{1}\right\rangle^{2}\left\langle 2 \ell_{2}\right\rangle^{2}[56]^{3}}{\langle 23\rangle\left\langle 3 \ell_{2}\right\rangle\left\langle\ell_{1} 2\right\rangle\left\langle\ell_{1} \ell_{2}\right\rangle\left\langle\ell_{2} \ell_{1}\right\rangle\left\langle 1\left|P_{456}\right| 4\right]\left\langle\ell_{2}\left|P_{456}\right| 6\right][45] P_{456}}\right. \\
& +\frac{\left\langle 2 \ell_{1}\right\rangle^{2}\left\langle 2 \ell_{2}\right\rangle^{2}\left\langle 1\left|P_{561}\right| \ell_{1}\right]^{2}\left\langle 1\left|P_{561}\right| \ell_{2}\right]^{2}}{\langle 23\rangle\left\langle 3 \ell_{2}\right\rangle\langle 56\rangle\langle 61\rangle\left\langle\ell_{1} 2\right\rangle\left\langle\ell_{2} \ell_{1}\right\rangle\left\langle 1\left|P_{561}\right| 4\right]\left\langle 5\left|P_{561}\right| \ell_{1}\right]\left[\ell_{1} \ell_{2}\right]\left[\ell_{2} 4\right] P_{561}} \\
& \left.+\frac{\left\langle 2 \ell_{1}\right\rangle^{2}\left\langle 2 \ell_{2}\right\rangle^{2}\left\langle\ell_{2} 4\right\rangle^{3}\left\langle 4\left|P_{61 \ell_{1}}\right| 6\right]^{2}\left[6 \ell_{1}\right]^{4}}{\langle 23\rangle\left\langle 3 \ell_{2}\right\rangle\langle 45\rangle\left\langle 4 \ell_{2}\right\rangle^{2}\left\langle\ell_{1} 2\right\rangle\left\langle\ell_{2} \ell_{1}\right\rangle\left\langle 5\left|P_{61 \ell_{1}}\right| \ell_{1}\right]\left\langle\ell_{2}\left|P_{61 \ell_{1}}\right| 6\right]\left[1 \ell_{1}\right][61]\left[\ell_{1} 6\right]^{2}\left(P_{61 \ell_{1}}^{2}\right)}\right\} .
\end{aligned}
$$

Therefore we concentrate just on the term $C_{23}^{(1)}$, and finally multiply by 2 , in order to get the coefficients of the proper functions. In particular, since this cut contains the contributions to both bubbles and three-mass triangle coefficients, we discuss these separately. 
In the following formulas, for this cut only, we define

$$
Q=\left(P_{61}^{2} / P_{23}^{2}\right) P_{23}+P_{61}
$$

and

$$
\left.\left.\mid \omega_{6}\right]=P_{23} P_{123} \mid 6\right]
$$

\section{Rational contribution from $C_{23}^{(1)}$}

After the $t$-integration, the rational term which will contribute to the bubble coefficients $I_{2: 2 ; 2}$ reads as a sum of six terms:

$$
\begin{aligned}
C_{23}^{(1, r a t)} & =C_{23}^{(1, r, 1)}+C_{23}^{(1, r, 2)}+C_{23}^{(1, r, 3)} \\
& +C_{23}^{(1, r, 4)}+C_{23}^{(1, r, 5)}+C_{23}^{(1, r, 6)} .
\end{aligned}
$$

For each of these we give the expression after the $t$-integration, the form as full derivative, and the residues.

The term $C_{23}^{(1, r, 1)}$

i) $t$-integrated formula:

$$
\begin{aligned}
C_{23}^{(1, r, 1)} & =\frac{\langle\ell d \ell\rangle[\ell d \ell]\left\langle 1\left|P_{56}\right| 3\right]^{2}\left\langle 1\left|P_{56}\right| \ell\right]^{2}[23][3 \ell]}{\langle 56\rangle\langle 61\rangle\left\langle 1\left|P_{56}\right| 4\right]\left\langle 5\left|P_{61}\right| \ell\right]\left\langle\ell\left|P_{23}\right| \ell\right]^{2}[2 \ell][4 \ell]\left(P_{561}^{2}\right)} \\
& +\frac{\langle\ell d \ell\rangle[\ell d \ell]\langle 23\rangle^{2}\left\langle 1\left|P_{56}\right| \ell\right]^{4}[23]^{3}[34]^{2}[3 \ell]}{\langle 56\rangle\langle 61\rangle\left\langle 1\left|P_{56}\right| 4\right]\left\langle 5\left|P_{61}\right| \ell\right]\left\langle\ell\left|P_{23}\right| \ell\right]^{2}[2 \ell][4 \ell]^{3}\left(P_{23}^{2}\right)^{2}\left(P_{561}^{2}\right)} \\
& -\frac{2\langle\ell d \ell\rangle[\ell d \ell]\langle 23\rangle\left\langle 1\left|P_{56}\right| 3\right]\left\langle 1\left|P_{56}\right| \ell\right]^{3}[23]^{2}[34][3 \ell]}{\langle 56\rangle\langle 61\rangle\left\langle 1\left|P_{56}\right| 4\right]\left\langle 5\left|P_{61}\right| \ell\right]\left\langle\ell\left|P_{23}\right| \ell\right]^{2}[2 \ell][4 \ell]^{2}\left(P_{23}^{2}\right)\left(P_{561}^{2}\right)}
\end{aligned}
$$

ii) full derivative:

$$
\begin{aligned}
C_{23}^{(1, r, 1)} & =\frac{[\ell d \ell]\left\langle d \ell \partial_{\ell}\right\rangle\langle 4 \ell\rangle\left\langle 1\left|P_{56}\right| 3\right]^{2}\left\langle 1\left|P_{56}\right| \ell\right]^{2}[23][3 \ell]}{\langle 56\rangle\langle 61\rangle\left\langle 1\left|P_{56}\right| 4\right]\left\langle 4\left|P_{23}\right| \ell\right]\left\langle 5\left|P_{61}\right| \ell\right]\left\langle\ell\left|P_{23}\right| \ell\right][2 \ell][4 \ell]\left(P_{561}^{2}\right)} \\
& +\frac{[\ell d \ell]\left\langle d \ell \partial_{\ell}\right\rangle\langle 23\rangle^{2}\langle 4 \ell\rangle\left\langle 1\left|P_{56}\right| \ell\right]^{4}[23]^{3}[34]^{2}[3 \ell]}{\langle 56\rangle\langle 61\rangle\left\langle 1\left|P_{56}\right| 4\right]\left\langle 4\left|P_{23}\right| \ell\right]\left\langle 5\left|P_{61}\right| \ell\right]\left\langle\ell\left|P_{23}\right| \ell\right][2 \ell][4 \ell]^{3}\left(P_{23}^{2}\right)^{2}\left(P_{561}^{2}\right)} \\
& -\frac{2[\ell d \ell]\left\langle d \ell \partial_{\ell}\right\rangle\langle 23\rangle\langle 4 \ell\rangle\left\langle 1\left|P_{56}\right| 3\right]\left\langle 1\left|P_{56}\right| \ell\right]^{3}[23]^{2}[34][3 \ell]}{\langle 56\rangle\langle 61\rangle\left\langle 1\left|P_{56}\right| 4\right]\left\langle 4\left|P_{23}\right| \ell\right]\left\langle 5\left|P_{61}\right| \ell\right]\left\langle\ell\left|P_{23}\right| \ell\right][2 \ell][4 \ell]^{2}\left(P_{23}^{2}\right)\left(P_{561}^{2}\right)}
\end{aligned}
$$


As one can see the higher pole $[4 \ell]^{n}$ in neutralized by the presence of $\langle 4 \ell\rangle$ in the corresponding numerator. Therefore, only single poles will give nonzero residues,

$$
\begin{aligned}
C_{23}^{(1, r, 1: s)} & =\sum_{i=1}^{4} \lim _{\ell \rightarrow \ell_{i}}\left[\ell \ell_{i}\right]\left\{\frac{\langle 4 \ell\rangle\left\langle 1\left|P_{56}\right| 3\right]^{2}\left\langle 1\left|P_{56}\right| \ell\right]^{2}[23][3 \ell]}{\langle 56\rangle\langle 61\rangle\left\langle 1\left|P_{56}\right| 4\right]\left\langle 4\left|P_{23}\right| \ell\right]\left\langle 5\left|P_{61}\right| \ell\right]\left\langle\ell\left|P_{23}\right| \ell\right][2 \ell][4 \ell]\left(P_{561}^{2}\right)}\right. \\
& +\frac{\langle 23\rangle^{2}\langle 4 \ell\rangle\left\langle 1\left|P_{56}\right| \ell\right]^{4}[23]^{3}[34]^{2}[3 \ell]}{\langle 56\rangle\langle 61\rangle\left\langle 1\left|P_{56}\right| 4\right]\left\langle 4\left|P_{23}\right| \ell\right]\left\langle 5\left|P_{61}\right| \ell\right]\left\langle\ell\left|P_{23}\right| \ell\right][2 \ell][4 \ell]^{3}\left(P_{23}^{2}\right)^{2}\left(P_{561}^{2}\right)} \\
& \left.-\frac{2\langle 23\rangle\langle 4 \ell\rangle\left\langle 1\left|P_{56}\right| 3\right]\left\langle 1\left|P_{56}\right| \ell\right]^{3}[23]^{2}[34][3 \ell]}{\langle 56\rangle\langle 61\rangle\left\langle 1\left|P_{56}\right| 4\right]\left\langle 4\left|P_{23}\right| \ell\right]\left\langle 5\left|P_{61}\right| \ell\right]\left\langle\ell\left|P_{23}\right| \ell\right][2 \ell][4 \ell]^{2}\left(P_{23}^{2}\right)\left(P_{561}^{2}\right)}\right\}
\end{aligned}
$$

with $\left.\left.\left.\mid \ell_{i}\right]=P_{23}|4\rangle, P_{61}|5\rangle, \mid 2\right], \mid 4\right]$ for $(i=1, \ldots, 4)$.

The term $C_{23}^{(1, r, 2)}$

i) $t$-integrated formula:

$$
\begin{aligned}
C_{23}^{(1, r, 2)} & =\frac{2\langle\ell d \ell\rangle[\ell d \ell]\langle 2 \ell\rangle\left\langle 1\left|P_{56}\right| 3\right]\left\langle 1\left|P_{56}\right| \ell\right]^{3}[23]^{2}[3 \ell]}{\langle 56\rangle\langle 61\rangle\left\langle 1\left|P_{56}\right| 4\right]\left\langle 5\left|P_{61}\right| \ell\right]\left\langle\ell\left|P_{23}\right| \ell\right]^{3}[2 \ell][4 \ell]\left(P_{561}^{2}\right)} \\
& -\frac{\langle\ell d \ell\rangle[\ell d \ell]\langle 23\rangle\langle 2 \ell\rangle\left\langle 1\left|P_{56}\right| \ell\right]^{4}[23]^{3}[34][3 \ell]}{\langle 56\rangle\langle 61\rangle\left\langle 1\left|P_{56}\right| 4\right]\left\langle 5\left|P_{61}\right| \ell\right]\left\langle\ell\left|P_{23}\right| \ell\right]^{3}[2 \ell][4 \ell]^{2}\left(P_{23}^{2}\right)\left(P_{561}^{2}\right)}
\end{aligned}
$$

ii) full derivative:

$$
\begin{aligned}
C_{23}^{(1, r, 2)} & =\frac{[\ell d \ell]\left\langle d \ell \partial_{\ell}\right\rangle\langle 2 \ell\rangle^{2}\left\langle 1\left|P_{56}\right| 3\right]\left\langle 1\left|P_{56}\right| \ell\right]^{3}[23]^{2}}{\langle 23\rangle\langle 56\rangle\langle 61\rangle\left\langle 1\left|P_{56}\right| 4\right]\left\langle 5\left|P_{61}\right| \ell\right]\left\langle\ell\left|P_{23}\right| \ell\right]^{2}[2 \ell][4 \ell]\left(P_{561}^{2}\right)} \\
& -\frac{[\ell d \ell]\left\langle d \ell \partial_{\ell}\right\rangle\langle 2 \ell\rangle^{2}\left\langle 1\left|P_{56}\right| \ell\right]^{4}[23]^{3}[34]}{2\langle 56\rangle\langle 61\rangle\left\langle 1\left|P_{56}\right| 4\right]\left\langle 5\left|P_{61}\right| \ell\right]\left\langle\ell\left|P_{23}\right| \ell\right]^{2}[2 \ell][4 \ell]^{2}\left(P_{23}^{2}\right)\left(P_{561}^{2}\right)}
\end{aligned}
$$

In this case we have both the residues of the simple poles,

$$
\begin{aligned}
C_{23}^{(1, r, 2: s)} & =\frac{\left\langle 1\left|P_{56}\right| 3\right][23]^{2}}{\langle 23\rangle\langle 56\rangle\langle 61\rangle\left(P_{561}^{2}\right)} \sum_{i=2}^{4} \lim _{\ell \rightarrow \ell_{i}}\left[\ell \ell_{i}\right] \frac{\langle 2 \ell\rangle^{2}\left\langle 1\left|P_{56}\right| \ell\right]^{3}}{\left\langle 1\left|P_{56}\right| 4\right]\left\langle 5\left|P_{61}\right| \ell\right]\left\langle\ell\left|P_{23}\right| \ell\right]^{2}[2 \ell][4 \ell]} \\
& -\frac{[23]^{3}[34]}{2\langle 56\rangle\langle 61\rangle\left\langle 1\left|P_{56}\right| 4\right]\left(P_{23}^{2}\right)\left(P_{561}^{2}\right)} \sum_{i=2}^{3} \lim _{\ell \rightarrow \ell_{i}}\left[\ell \ell_{i}\right] \frac{\langle 2 \ell\rangle^{2}\left\langle 1\left|P_{56}\right| \ell\right]^{4}}{\left\langle 5\left|P_{61}\right| \ell\right]\left\langle\ell\left|P_{23}\right| \ell\right]^{2}[2 \ell][4 \ell]^{2}}
\end{aligned}
$$

and the residue from a double pole,

$$
\left.C_{23}^{(1, r, 2: d)}=-\frac{[23]^{3}[34]\langle 24\rangle^{2}}{2\langle 56\rangle\langle 61\rangle\left\langle 1\left|P_{56}\right| 4\right]\left(P_{23}^{2}\right)\left(P_{561}^{2}\right)} P_{2}[\mid 4], L_{1}^{I I: C_{23}}, L_{2}^{I I: C_{23}}\right],
$$

with

$$
\begin{aligned}
& L_{1}^{I I: C_{23}}=\left\{P_{561}|1\rangle, P_{561}|1\rangle, P_{561}|1\rangle, P_{561}|1\rangle\right\} \\
& \left.L_{2}^{I I: C_{23}}=\left\{P_{561}|5\rangle, P_{23}|4\rangle, P_{23}|4\rangle, \mid 2\right]\right\} .
\end{aligned}
$$


The term $C_{23}^{(1, r, 3)}$

i) $t$-integrated formula:

$$
C_{23}^{(1, r, 3)}=\frac{\langle\ell d \ell\rangle[\ell d \ell]\langle 2 \ell\rangle^{2}\left\langle 1\left|P_{56}\right| \ell\right]^{4}[23]^{3}[3 \ell]}{\langle 56\rangle\langle 61\rangle\left\langle 1\left|P_{56}\right| 4\right]\left\langle 5\left|P_{61}\right| \ell\right]\left\langle\ell\left|P_{23}\right| \ell\right]^{4}[2 \ell][4 \ell]\left(P_{561}^{2}\right)}
$$

ii) full derivative:

$$
C_{23}^{(1, r, 3)}=\frac{[\ell d \ell]\left\langle d \ell \partial_{\ell}\right\rangle\langle 2 \ell\rangle^{3}\left\langle 1\left|P_{56}\right| \ell\right]^{4}[23]^{3}}{3\langle 23\rangle\langle 56\rangle\langle 61\rangle\left\langle 1\left|P_{56}\right| 4\right]\left\langle 5\left|P_{61}\right| \ell\right]\left\langle\ell\left|P_{23}\right| \ell\right]^{3}[2 \ell][4 \ell]\left(P_{561}^{2}\right)}
$$

There are only simple poles. Therefore,

$$
C_{23}^{(1, r, 3: s)}=\frac{[23]^{3}}{3\langle 23\rangle\langle 56\rangle\langle 61\rangle\left\langle 1\left|P_{56}\right| 4\right]\left(P_{561}^{2}\right)} \sum_{i=2}^{4} \lim _{\ell \rightarrow \ell_{i}}\left[\ell \ell_{i}\right] \frac{\langle 2 \ell\rangle^{3}\left\langle 1\left|P_{56}\right| \ell\right]^{4}}{\left\langle 5\left|P_{61}\right| \ell\right]\left\langle\ell\left|P_{23}\right| \ell\right]^{3}[2 \ell][4 \ell]}
$$

The term $C_{23}^{(1, r, 4)}$

i) $t$-integrated formula:

$$
\begin{aligned}
C_{23}^{(1, r, 4)}= & -\frac{\langle\ell d \ell\rangle[\ell d \ell]\langle 23\rangle\langle 41\rangle^{2}\left\langle 4\left|P_{23}\right| \ell\right][3 \ell]^{3}[61][6 \ell]^{2}}{\langle 45\rangle\left\langle 5\left|P_{61}\right| \ell\right]\left\langle\ell\left|P_{23}\right| \ell\right]^{2}[1 \ell][2 \ell]\left[\ell\left|P_{23} Q\right| \ell\right]\left[\ell \omega_{6}\right]} \\
- & \frac{2\langle\ell d \ell\rangle[\ell d \ell]\langle 41\rangle\langle 2|Q| \ell]\left\langle 4\left|P_{23}\right| \ell\right]^{2}[3 \ell]^{2}[6 \ell]^{3}\left(P_{23}^{2}\right)}{\langle 45\rangle\left\langle 5\left|P_{61}\right| \ell\right]\left\langle\ell\left|P_{23}\right| \ell\right]^{2}[1 \ell][2 \ell]\left[\ell\left|P_{23} Q\right| \ell\right]^{2}\left[\ell \omega_{6}\right]} \\
- & \frac{\langle\ell d \ell\rangle[\ell d \ell]\langle 2|Q| \ell]\langle 4|Q| \ell]\left\langle 4\left|P_{23}\right| \ell\right]^{2}[3 \ell]^{2}[6 \ell]^{4}\left(P_{23}^{2}\right)^{2}}{\langle 45\rangle\left\langle 5\left|P_{61}\right| \ell\right]\left\langle\ell\left|P_{23}\right| \ell\right]^{2}[1 \ell][2 \ell][61]\left[\ell\left|P_{23} Q\right| \ell\right]^{3}\left[\ell \omega_{6}\right]}
\end{aligned}
$$

ii) full derivative:

$$
C_{23}^{(1, r, 4)}=[\ell d \ell]\left\langle d \ell \partial_{\ell}\right\rangle \mathcal{I}^{(4)}
$$




$$
\begin{aligned}
\mathcal{I}^{(4)} & =-\frac{\langle 2 \ell\rangle\langle 41\rangle^{2}\left\langle 4\left|P_{23}\right| \ell\right][3 \ell]^{2}[61][6 \ell]^{2}[a b]^{2}}{\langle 45\rangle\left\langle 5\left|P_{61}\right| \ell\right]\left\langle\ell\left|P_{23}\right| \ell\right][1 \ell][2 \ell]\left[\eta_{1} \ell\right]\left[\eta_{2} \ell\right]\left[b\left|P_{23} Q\right| b\right]\left[\ell \omega_{6}\right]} \\
& -\frac{2\langle 41\rangle\left\langle\eta_{1} \ell\right\rangle\langle 2|Q| \ell]\left\langle 4\left|P_{23}\right| \ell\right]^{2}[6 \ell]^{3}[a b]^{4}\left[\eta_{1} 3\right]^{2}\left(P_{23}^{2}\right)}{\langle 45\rangle\left\langle 5\left|P_{61}\right| \ell\right]\left\langle\ell\left|P_{23}\right| \ell\right]\left\langle\eta_{1}\left|P_{23}\right| \ell\right][1 \ell][2 \ell]\left[\eta_{1} \ell\right]^{2}\left[\eta_{1} \eta_{2}\right]^{2}\left[b\left|P_{23} Q\right| b\right]^{2}\left[\ell \omega_{6}\right]} \\
& -\frac{2\langle 41\rangle\left\langle\eta_{2} \ell\right\rangle\langle 2|Q| \ell]\left\langle 4\left|P_{23}\right| \ell\right]^{2}\left[3 \eta_{2}\right]^{2}[6 \ell]^{3}[a b]^{4}\left(P_{23}^{2}\right)}{\langle 45\rangle\left\langle 5\left|P_{61}\right| \ell\right]\left\langle\ell\left|P_{23}\right| \ell\right]\left\langle\eta_{2}\left|P_{23}\right| \ell\right][1 \ell][2 \ell]\left[\eta_{1} \eta_{2}\right]^{2}\left[\eta_{2} \ell\right]^{2}\left[b\left|P_{23} Q\right| b\right]^{2}\left[\ell \omega_{6}\right]} \\
& -\frac{4\langle 1 \ell\rangle\langle 41\rangle\langle 2|Q| \ell]\left\langle 4\left|P_{23}\right| \ell\right]^{2}\left[3 \eta_{2}\right][6 \ell]^{3}[a b]^{4}\left[\eta_{1} 3\right]\left(P_{23}^{2}\right)}{\langle 45\rangle\left\langle 1\left|P_{23}\right| \ell\right]\left\langle 5\left|P_{61}\right| \ell\right]\left\langle\ell\left|P_{23}\right| \ell\right|[1 \ell][2 \ell]\left[\eta_{1} \ell\right]\left[\eta_{1} \eta_{2}\right]^{2}\left[\eta_{2} \ell\right]\left[b\left|P_{23} Q\right| b\right]^{2}\left[\ell \omega_{6}\right]} \\
& -\frac{\left\langle\eta_{1} \ell\right\rangle\langle 2|Q| \ell]\langle 4|Q| \ell]\left\langle 4\left|P_{23}\right| \ell\right]^{2}[3 \ell]^{2}[6 \ell][a b]^{6}\left[\eta_{1} 6\right]^{3}\left(P_{23}^{2}\right)^{2}}{\langle 45\rangle\left\langle 5\left|P_{61}\right| \ell\right]\left\langle\ell\left|P_{23}\right| \ell\right]\left\langle\eta_{1}\left|P_{23}\right| \ell\right][1 \ell][2 \ell][61]\left[\eta_{1} \ell\right]^{3}\left[\eta_{1} \eta_{2}\right]^{3}\left[b\left|P_{23} Q\right| b\right]^{3}\left[\ell \omega_{6}\right]} \\
& -\frac{\left\langle\eta_{2} \ell\right\rangle\langle 2|Q| \ell]\langle 4|Q| \ell]\left\langle 4\left|P_{23}\right| \ell\right]^{2}[3 \ell]^{2}[6 \ell]\left[6 \eta_{2}\right]^{3}[a b]^{6}\left(P_{23}^{2}\right)^{2}}{\langle 45\rangle\left\langle 5\left|P_{61}\right| \ell\right]\left\langle\ell\left|P_{23}\right| \ell\right]\left\langle\eta_{2}\left|P_{23}\right| \ell\right][1 \ell][2 \ell][61]\left[\eta_{1} \eta_{2}\right]^{3}\left[\eta_{2} \ell\right]^{3}\left[b\left|P_{23} Q\right| b\right]^{3}\left[\ell \omega_{6}\right]} \\
& -\frac{3\left\langle\eta_{2} \ell\right\rangle\langle 2|Q| \ell]\langle 4|Q| \ell]\left\langle 4\left|P_{23}\right| \ell\right]^{2}[3 \ell]^{2}[6 \ell]\left[6 \eta_{2}\right]^{2}[a b]^{6}\left[\eta_{1} 6\right]\left(P_{23}^{2}\right)^{2}}{\langle 45\rangle\left\langle 5\left|P_{61}\right| \ell\right]\left\langle\ell\left|P_{23}\right| \ell\right]\left\langle\eta_{2}\left|P_{23}\right| \ell\right][1 \ell][2 \ell][61]\left[\eta_{1} \ell\right]\left[\eta_{1} \eta_{2}\right]^{3}\left[\eta_{2} \ell\right]^{2}\left[b\left|P_{23} Q\right| b\right]^{3}\left[\ell \omega_{6}\right]} \\
& -\frac{3\left\langle\eta_{1} \ell\right\rangle\langle 2|Q| \ell]\langle 4|Q| \ell]\left\langle 4\left|P_{23}\right| \ell\right]^{2}[3 \ell]^{2}[6 \ell]\left[6 \eta_{2}\right][a b]^{6}\left[\eta_{1} 6\right]^{2}\left(P_{23}^{2}\right)^{2}}{\langle 45\rangle\left\langle 5\left|P_{61}\right| \ell\right]\left\langle\ell\left|P_{23}\right| \ell\right]\left\langle\eta_{1}\left|P_{23}\right| \ell\right][1 \ell][2 \ell][61]\left[\eta_{1} \ell\right]^{2}\left[\eta_{1} \eta_{2}\right]^{3}\left[\eta_{2} \ell\right]\left[b\left|P_{23} Q\right| b\right]^{3}\left[\ell \omega_{6}\right]} .
\end{aligned}
$$

The term $C_{23}^{(1, r, 5)}$

i) $t$-integrated formula:

$$
\begin{aligned}
C_{23}^{(1, r, 5)} & =-2 \frac{\langle\ell d \ell\rangle[\ell d \ell]\langle 23\rangle\langle 41\rangle\langle 4 \ell\rangle\left\langle 4\left|P_{23}\right| \ell\right][3 \ell]^{3}[6 \ell]^{3}\left(P_{23}^{2}\right)}{\langle 45\rangle\left\langle 5\left|P_{61}\right| \ell\right|\left\langle\ell\left|P_{23}\right| \ell\right]^{3}[1 \ell][2 \ell]\left[\ell\left|P_{23} Q\right| \ell\right]\left[\ell \omega_{6}\right]} \\
& -\frac{\langle\ell d \ell\rangle[\ell d \ell]\langle 4 \ell\rangle\langle 2|Q| \ell]\left\langle 4\left|P_{23}\right| \ell\right]^{2}[3 \ell]^{2}[6 \ell]^{4}\left(P_{23}^{2}\right)^{2}}{\langle 45\rangle\left\langle 5\left|P_{61}\right| \ell\right]\left\langle\ell\left|P_{23}\right| \ell\right]^{3}[1 \ell][2 \ell][61]\left[\ell\left|P_{23} Q\right| \ell\right]^{2}\left[\ell \omega_{6}\right]} \\
& +\frac{\langle\ell d \ell\rangle[\ell d \ell]\langle 1 \ell\rangle\langle 21\rangle\left\langle 1\left|P_{23}\right| \ell\right][3 \ell]^{2}[56]^{3}\left(P_{23}^{2}\right)}{\left\langle 1\left|P_{456}\right| 4\right]\left\langle\ell\left|P_{23}\right| \ell\right]^{3}[2 \ell][45]\left[\ell \omega_{6}\right]\left(P_{123}^{2}\right)}
\end{aligned}
$$

ii) full derivative:

$$
C_{23}^{(1, r, 5)}=[\ell d \ell]\left\langle d \ell \partial_{\ell}\right\rangle \mathcal{I}^{(5)}
$$




$$
\begin{aligned}
\mathcal{I}^{(5)} & =-\frac{\langle 23\rangle\langle 41\rangle\langle 4 \ell\rangle^{2}[3 \ell]^{3}[6 \ell]^{3}[a b]^{2}\left(P_{23}^{2}\right)}{\langle 45\rangle\left\langle 5\left|P_{61}\right| \ell\right]\left\langle\ell\left|P_{23}\right| \ell\right]^{2}[1 \ell][2 \ell]\left[\eta_{1} \ell\right]\left[\eta_{2} \ell\right]\left[b\left|P_{23} Q\right| b\right]\left[\ell \omega_{6}\right]} \\
& -\frac{\left\langle\eta_{1} \ell\right\rangle^{2}\langle 2|Q| \ell]\left\langle 4\left|P_{23}\right| \ell\right]^{3}[6 \ell]^{4}[a b]^{4}\left[\eta_{1} 3\right]^{2}\left(P_{23}^{2}\right)^{2}}{2\langle 45\rangle\left\langle 5\left|P_{61}\right| \ell\right]\left\langle\ell\left|P_{23}\right| \ell\right]^{2}\left\langle\eta_{1}\left|P_{23}\right| \ell\right]^{2}[1 \ell][2 \ell][61]\left[\eta_{1} \ell\right]^{2}\left[\eta_{1} \eta_{2}\right]^{2}\left[b\left|P_{23} Q\right| b\right]^{2}\left[\ell \omega_{6}\right]} \\
& -\frac{\left\langle 4 \eta_{1}\right\rangle\left\langle\eta_{1} \ell\right\rangle\langle 2|Q| \ell]\left\langle 4\left|P_{23}\right| \ell\right]^{2}[6 \ell]^{4}[a b]^{4}\left[\eta_{1} 3\right]^{2}\left(P_{23}^{2}\right)^{2}}{\langle 45\rangle\left\langle 5\left|P_{61}\right| \ell\right]\left\langle\ell\left|P_{23}\right| \ell\right]\left\langle\eta_{1}\left|P_{23}\right| \ell\right]^{2}[1 \ell][2 \ell][61]\left[\eta_{1} \ell\right]^{2}\left[\eta_{1} \eta_{2}\right]^{2}\left[b\left|P_{23} Q\right| b\right]^{2}\left[\ell \omega_{6}\right]} \\
& -\frac{\left\langle\eta_{2} \ell\right\rangle^{2}\langle 2|Q| \ell]\left\langle 4\left|P_{23}\right| \ell\right]^{3}\left[3 \eta_{2}\right]^{2}[6 \ell]^{4}[a b]^{4}\left(P_{23}^{2}\right)^{2}}{2\langle 45\rangle\left\langle 5\left|P_{61}\right| \ell\right]\left\langle\ell\left|P_{23}\right| \ell\right]^{2}\left\langle\eta_{2}\left|P_{23}\right| \ell\right]^{2}[1 \ell][2 \ell][61]\left[\eta_{1} \eta_{2}\right]^{2}\left[\eta_{2} \ell\right]^{2}\left[b\left|P_{23} Q\right| b\right]^{2}\left[\ell \omega_{6}\right]} \\
& -\frac{\left\langle 4 \eta_{2}\right\rangle\left\langle\eta_{2} \ell\right\rangle\langle 2|Q| \ell]\left\langle 4\left|P_{23}\right| \ell\right]^{2}\left[3 \eta_{2}\right]^{2}[6 \ell]^{4}[a b]^{4}\left(P_{23}^{2}\right)^{2}}{\langle 45\rangle\left\langle 5\left|P_{61}\right| \ell\right]\left\langle\ell\left|P_{23}\right| \ell\right]\left\langle\eta_{2}\left|P_{23}\right| \ell\right]^{2}[1 \ell][2 \ell][61]\left[\eta_{1} \eta_{2}\right]^{2}\left[\eta_{2} \ell\right]^{2}\left[b\left|P_{23} Q\right| b\right]^{2}\left[\ell \omega_{6}\right]} \\
& -\frac{\langle 4 \ell\rangle^{2}\langle 2|Q| \ell]\left\langle 4\left|P_{23}\right| \ell\right]\left[3 \eta_{2}\right][6 \ell]^{4}[a b]^{4}\left[\eta_{1} 3\right]\left(P_{23}^{2}\right)^{2}}{\langle 45\rangle\left\langle 5\left|P_{61}\right| \ell\right]\left\langle\ell\left|P_{23}\right| \ell\right]^{2}[1 \ell][2 \ell][61]\left[\eta_{1} \ell\right]\left[\eta_{1} \eta_{2}\right]^{2}\left[\eta_{2} \ell\right]\left[b\left|P_{23} Q\right| b\right]^{2}\left[\ell \omega_{6}\right]} \\
+ & \frac{\langle 1 \ell\rangle^{2}\langle 21\rangle[3 \ell]^{2}[56]^{3}\left(P_{23}^{2}\right)}{2\left\langle 1\left|P_{456}\right| 4\right]\left\langle\ell\left|P_{23}\right| \ell\right]^{2}[2 \ell][45]\left[\ell \omega_{6}\right]\left(P_{123}^{2}\right)} .
\end{aligned}
$$

The above expression contains single and double poles. The residues of the single poles will be read off later; for the total rational contribution, we consider here only the terms having double poles, $\left\langle\eta_{1}\left|P_{23}\right| \ell\right]^{2}$ and $\left\langle\eta_{2}\left|P_{23}\right| \ell\right]^{2}$ :

$$
\begin{gathered}
C_{23}^{(1, r, 5: d)}=[\ell d \ell]\left\langle d \ell \partial_{\ell}\right\rangle \mathcal{I}^{(5: d)} \\
\mathcal{I}^{(5: d)=}=\frac{\left\langle\eta_{1} \ell\right\rangle^{2}\langle 2|Q| \ell]\left\langle 4\left|P_{23}\right| \ell\right]^{3}[6 \ell]^{4}[a b]^{4}\left[\eta_{1} 3\right]^{2}\left(P_{23}^{2}\right)^{2}}{2\langle 45\rangle\left\langle 5\left|P_{61}\right| \ell\right]\left\langle\ell\left|P_{23}\right| \ell\right]^{2}\left\langle\eta_{1}\left|P_{23}\right| \ell\right]^{2}[1 \ell][2 \ell][61]\left[\eta_{1} \ell\right]^{2}\left[\eta_{1} \eta_{2}\right]^{2}\left[b\left|P_{23} Q\right| b\right]^{2}\left[\ell \omega_{6}\right]} \\
\\
-\frac{\left\langle 4 \eta_{1}\right\rangle\left\langle\eta_{1} \ell\right\rangle\langle 2|Q| \ell]\left\langle 4\left|P_{23}\right| \ell\right]^{2}[6 \ell]^{4}[a b]^{4}\left[\eta_{1} 3\right]^{2}\left(P_{23}^{2}\right)^{2}}{\langle 45\rangle\left\langle 5\left|P_{61}\right| \ell\right]\left\langle\ell\left|P_{23}\right| \ell\right]\left\langle\eta_{1}\left|P_{23}\right| \ell\right]^{2}[1 \ell][2 \ell][61]\left[\eta_{1} \ell\right]^{2}\left[\eta_{1} \eta_{2}\right]^{2}\left[b\left|P_{23} Q\right| b\right]^{2}\left[\ell \omega_{6}\right]} \\
+\left\{\eta_{1} \rightarrow \eta_{2}\right\}
\end{gathered}
$$

The residues in this case give

$$
\begin{aligned}
C_{23}^{(1, r, 5: d)}= & \frac{[a b]^{4}\left[\eta_{1} 3\right]^{2}\left\langle\eta_{1}\left|P_{23}\right| \eta_{1}\right]^{2}}{2\langle 45\rangle[61]\left[\eta_{1} \eta_{2}\right]^{2}\left[b\left|P_{23} Q\right| b\right]^{2}} \widetilde{P}_{2}\left[P_{23}\left|\eta_{1}\right\rangle, L_{1}^{I I: C_{23}}, L_{2}^{I I: C_{23}}\right] \\
& +\frac{\left\langle 4 \eta_{1}\right\rangle[a b]^{4}\left[\eta_{1} 3\right]^{2}\left\langle\eta_{1}\left|P_{23}\right| \eta_{1}\right]\left(P_{23}^{2}\right)}{\langle 45\rangle[61]\left[\eta_{1} \eta_{2}\right]^{2}\left[b\left|P_{23} Q\right| b\right]^{2}} \widetilde{P}_{2}\left[P_{23}\left|\eta_{1}\right\rangle, M_{1}^{I I: C_{23}}, M_{2}^{I I: C_{23}}\right] \\
& +\left\{\eta_{1} \leftrightarrow \eta_{2}\right\},
\end{aligned}
$$


with

$$
\begin{aligned}
L_{1}^{I I: C_{23}} & \left.\left.\left.\left.=\left\{Q|2\rangle, P_{23}|4\rangle, P_{23}|4\rangle, P_{23}|4\rangle, \mid 6\right], \mid 6\right], \mid 6\right], \mid 6\right]\right\} \\
L_{2}^{I I: C_{23}} & \left.\left.\left.\left.\left.\left.\left.=\left\{P_{61}|5\rangle, \mid 1\right], \mid 2\right], \mid \eta_{1}\right], \mid \eta_{1}\right], \mid \eta_{1}\right], \mid \eta_{1}\right], \mid \omega_{6}\right]\right\} \\
M_{1}^{I I: C_{23}} & \left.\left.\left.\left.=\left\{Q|2\rangle, P_{23}|4\rangle, P_{23}|4\rangle, \mid 6\right], \mid 6\right], \mid 6\right], \mid 6\right]\right\} \\
M_{2}^{I I: C_{23}} & \left.\left.\left.\left.\left.\left.=\left\{P_{61}|5\rangle, \mid 1\right], \mid 2\right], \mid \eta_{1}\right], \mid \eta_{1}\right], \mid \eta_{1}\right], \mid \omega_{6}\right]\right\}
\end{aligned}
$$

since we used $\left.|\ell\rangle=P_{23} \mid \eta_{1}\right]$.

The term $C_{23}^{(1, r, 6)}$

i) $t$-integrated formula:

$$
\begin{aligned}
C_{23}^{(1, r, 6)} & =-\frac{\langle\ell d \ell\rangle[\ell d \ell]\langle 23\rangle\langle 4 \ell\rangle^{2}\left\langle 4\left|P_{23}\right| \ell\right][3 \ell]^{3}[6 \ell]^{4}\left(P_{23}^{2}\right)^{2}}{\langle 45\rangle\left\langle 5\left|P_{61}\right| \ell\right]\left\langle\ell\left|P_{23}\right| \ell\right]^{4}[1 \ell][2 \ell][61]\left[\ell\left|P_{23} Q\right| \ell\right]\left[\ell \omega_{6}\right]} \\
& +\frac{\langle\ell d \ell\rangle[\ell d \ell]\langle 1 \ell\rangle^{2}\langle 23\rangle\left\langle 1\left|P_{23}\right| \ell\right][3 \ell]^{3}[56]^{3}\left(P_{23}^{2}\right)}{\left\langle 1\left|P_{456}\right| 4\right]\left\langle\ell\left|P_{23}\right| \ell\right]^{4}[2 \ell][45]\left[\ell \omega_{6}\right]\left(P_{123}^{2}\right)}
\end{aligned}
$$

ii) full derivative:

$$
\begin{gathered}
C_{23}^{(1, r, 6)}=[\ell d \ell]\left\langle d \ell \partial_{\ell}\right\rangle \mathcal{I}^{(6)} \\
\mathcal{I}^{(6)}=-\frac{\langle 23\rangle\langle 4 \ell\rangle^{3}[3 \ell]^{3}[6 \ell]^{4}[a b]^{2}\left(P_{23}^{2}\right)^{2}}{3\langle 45\rangle\left\langle 5\left|P_{61}\right| \ell\right]\left\langle\ell\left|P_{23}\right| \ell\right]^{3}[1 \ell][2 \ell][61]\left[\eta_{1} \ell\right]\left[\eta_{2} \ell\right]\left[b\left|P_{23} Q\right| b\right]\left[\ell \omega_{6}\right]} \\
+\frac{\langle 1 \ell\rangle^{3}\langle 23\rangle[3 \ell]^{3}[56]^{3}\left(P_{23}^{2}\right)}{3\left\langle 1\left|P_{456}\right| 4\right]\left\langle\ell\left|P_{23}\right| \ell\right]^{3}[2 \ell][45]\left[\ell \omega_{6}\right]\left(P_{123}^{2}\right)}
\end{gathered}
$$

Given the expressions of $C_{23}^{(1, r, 4)}, C_{23}^{(1, r, 5)}$ and $C_{23}^{(1, r, 6)}$, in eqs.(5.55), (5.60), and (5.65), their combined contribution can be written as,

$$
C_{23}^{(1, r, 4,5,6)}=[\ell d \ell]\left\langle d \ell \partial_{\ell}\right\rangle\left\{\mathcal{I}^{(4)}+\mathcal{I}^{(5)}+\mathcal{I}^{(6)}\right\}
$$

Therefore, the sum of residues of their single poles will give

$$
C_{23}^{(1, r, 4,5,6: s)}=\sum_{j=1}^{10} \lim _{\ell \rightarrow \ell_{j}}\left[\ell \ell_{j}\right]\left\{\mathcal{I}^{(4)}+\mathcal{I}^{(5)}+\mathcal{I}^{(6)}\right\}
$$

with

$$
\left.\left.\left.\left.\left.\left.\left.\mid \ell_{j}\right]=P_{61}|5\rangle, \mid 1\right], \mid 2\right], \mid 4\right], \mid \eta_{1}\right], \mid \eta_{2}\right], \mid \omega_{6}\right], P_{23}|1\rangle, P_{23}\left|\eta_{1}\right\rangle, P_{23}\left|\eta_{2}\right\rangle \quad(j=1, \ldots, 10)
$$

The coefficient of the bubble $I_{2: 2 ; 2}$ is given by adding eqs. (5.44), (5.47), (5.48), (5.52), (5.67), (5.61), and multiplying by 2 :

$$
c_{2: 2 ; 2}=2\left(C_{23}^{(1, r, 1: s)}+C_{23}^{(1, r, 2: s)}+C_{23}^{(1, r, 2: d)}+C_{23}^{(1, r, 3: s)}+C_{23}^{(1, r, 3,4,5,6: s)}+C_{23}^{(1, r, 5: d)}\right) .
$$




\section{3-Mass-Triangle contribution from $C_{23}^{(1)}$}

After the $t$-integration the contribution to the three-mass-triangle coefficient reads:

$$
C_{23}^{(1,3 m)}=C_{23}^{(1,3 m, 1)}+C_{23}^{(1,3 m, 2)}+C_{23}^{(1,3 m, 3)} .
$$

Unlike in the case presented in Section 4, here we make use of the functions defined in Appendix $\mathrm{C}$ to write down answers directly and compactly.

The term $C_{23}^{(1,3 m, 1)}$

i) after $t$-integration

$$
C_{23}^{(1,3 m, 1)}=-\frac{\langle 41\rangle^{2}[61]}{\langle 45\rangle} \frac{\langle\ell d \ell\rangle[\ell d \ell]\langle 2|Q| \ell]\left\langle 4\left|P_{23}\right| \ell\right][3 \ell]^{2}[6 \ell]^{2}}{\langle\ell|Q| \ell]\left\langle\ell\left|P_{23}\right| \ell\right]\left[\ell\left|P_{23} Q\right| \ell\right]\left\langle 5\left|P_{61}\right| \ell\right][1 \ell][2 \ell]\left[\omega_{6} \ell\right]}
$$

ii) triangle coefficient:

$$
\begin{aligned}
C_{23}^{(1,3 m, 1)} & =-\frac{\langle 41\rangle^{2}[61]}{\langle 45\rangle} \widetilde{C}_{3}^{I I}\left[L_{a}, L_{b}^{I I}, P_{23}, Q\right] \\
L_{a} & \left.\left.\left.\left.=\left\{Q|2\rangle, P_{23}|4\rangle, \mid 3\right], \mid 3\right], \mid 6\right], \mid 6\right]\right\} \\
L_{b}^{I I} & \left.\left.\left.\left.=\left\{P_{61}|5\rangle, \mid 1\right], \mid 2\right], \mid \omega_{6}\right], \mid \eta\right]\right\}
\end{aligned}
$$

The term $C_{23}^{(1,3 m, 2)}$

i) after $t$-integration

$$
C_{23}^{(1,3 m, 2)}=-\frac{2\langle 41\rangle\left(P_{23}^{2}\right)}{\langle 45\rangle} \frac{\langle\ell d \ell\rangle[\ell d \ell]\langle 2|Q| \ell]\langle 4|Q| \ell]\left\langle 4\left|P_{23}\right| \ell\right][3 \ell]^{2}[6 \ell]^{3}}{\langle\ell|Q| \ell]\left\langle\ell\left|P_{23}\right| \ell\right]\left[\ell\left|P_{23} Q\right| \ell\right]^{2}\left\langle 5\left|P_{61}\right| \ell\right][1 \ell][2 \ell]\left[\omega_{6} \ell\right]}
$$

ii) triangle coefficient:

$$
\begin{aligned}
C_{23}^{(1,3 m, 2)} & =-\frac{2\langle 41\rangle\left(P_{23}^{2}\right)}{\langle 45\rangle} \widetilde{C}_{3}^{I I I}\left[L_{a}, L_{b, 1}^{I I I}, L_{b, 2}^{I I I}, P_{23}, Q\right] \\
L_{a} & \left.\left.\left.\left.\left.=\left\{Q|2\rangle, Q|4\rangle, P_{23}|4\rangle, \mid 3\right], \mid 3\right], \mid 6\right], \mid 6\right], \mid 6\right]\right\} \\
L_{b, 1}^{I I I} & \left.\left.\left.\left.\left.\left.=\left\{P_{61}|5\rangle, \mid 1\right], \mid 2\right], \mid \omega_{6}\right], \mid \eta\right], \mid \eta_{2}\right], \mid \eta_{2}\right]\right\} \\
L_{b, 2}^{I I I} & \left.\left.\left.\left.\left.\left.=\left\{P_{61}|5\rangle, \mid 1\right], \mid 2\right], \mid \omega_{6}\right], \mid \eta\right], \mid \eta_{1}\right], \mid \eta_{1}\right]\right\}
\end{aligned}
$$

The term $C_{23}^{(1,3 m, 3)}$ 
i) after $t$-integration

$$
C_{23}^{(1,3 m, 3)}=-\frac{\left(P_{23}^{2}\right)^{2}}{\langle 45\rangle[61]} \frac{\langle\ell d \ell\rangle[\ell d \ell]\langle 2|Q| \ell]\langle 4|Q| \ell]^{2}\left\langle 4\left|P_{23}\right| \ell\right][3 \ell]^{2}[6 \ell]^{4}}{\langle\ell|Q| \ell]\left\langle\ell\left|P_{23}\right| \ell\right]\left[\ell\left|P_{23} Q\right| \ell\right]^{3}\left\langle 5\left|P_{61}\right| \ell\right][1 \ell][2 \ell]\left[\omega_{6} \ell\right]}
$$

ii) triangle coefficient:

$$
\begin{gathered}
C_{23}^{(1,3 m, 3)}=-\frac{\left(P_{23}^{2}\right)^{2}}{\langle 45\rangle[61]} \widetilde{C}_{3}^{I V}\left[L_{a}, L_{b, 1}^{I V}, L_{b, 2}^{I V}, P_{23}, Q\right] \\
\left.\left.\left.\left.\left.\left.L_{a}=\left\{Q|2\rangle, Q|4\rangle, Q|4\rangle, P_{23}|4\rangle, \mid 3\right], \mid 3\right], \mid 6\right], \mid 6\right], \mid 6\right], \mid 6\right]\right\} \\
\left.\left.\left.\left.\left.\left.\left.L_{b, 1}^{I V}=\left\{P_{61}|5\rangle, \mid 1\right], \mid 2\right], \mid \omega_{6}\right], \mid \eta\right], \mid \eta_{2}\right], \mid \eta_{2}\right], \mid \eta_{2}\right]\right\} \\
\left.\left.\left.\left.\left.\left.\left.L_{b, 2}^{I V}=\left\{P_{61}|5\rangle, \mid 1\right], \mid 2\right], \mid \omega_{6}\right], \mid \eta\right], \mid \eta_{1}\right], \mid \eta_{1}\right], \mid \eta_{1}\right]\right\}
\end{gathered}
$$

Finally, the coefficient of the thee-mass triangle $I_{3: 2: 2 ; 2}$ can be obtained by taking the sum of (5.72), (5.75), and (5.78) and multiplying the result by 2 :

$$
c_{3: 2: 2 ; 2}=2\left(C_{23}^{(1,3 m, 1)}+C_{23}^{(1,3 m, 2)}+C_{23}^{(1,3 m, 3)}\right)
$$

\section{5. $C u t C_{61}$}

This cut has contributions from three-mass triangle, so the result will be more complicated. Also since it is the same three-mass triangle function as in cut $C_{23}$, we can use it as an independent check for this coefficient.

The cut is given by

$$
\begin{aligned}
C_{61}= & \int d \mu\left[A\left(\ell_{1}^{+}, 6^{+}, 1^{-}, \ell_{2}^{-}\right) A\left(\ell_{2}^{+}, 2^{-}, 3^{+}, 4^{-}, 5^{+}, \ell_{1}^{-}\right)\right. \\
& \left.+A\left(\ell_{1}^{-}, 6^{+}, 1^{-}, \ell_{2}^{+}\right) A\left(\ell_{2}^{-}, 2^{-}, 3^{+}, 4^{-}, 5^{+}, \ell_{1}^{+}\right)\right] \\
= & -\frac{2\left\langle 1 \ell_{1}\right\rangle^{2}\left\langle 1 \ell_{2}\right\rangle\langle 42\rangle^{4}\left[5 \ell_{1}\right]\left[5 \ell_{2}\right]^{2}}{\langle 23\rangle\langle 34\rangle\langle 61\rangle\left\langle 6 \ell_{1}\right\rangle\left\langle\ell_{2} \ell_{1}\right\rangle\left\langle 2\left|P_{234}\right| 5\right]\left\langle 4\left|P_{234}\right| \ell_{2}\right]\left[\ell_{1} \ell_{2}\right]\left(P_{234}^{2}\right)} \\
+ & \frac{2\left\langle 1 \ell_{1}\right\rangle^{2}\left\langle 1 \ell_{2}\right\rangle\left\langle 2 \ell_{1}\right\rangle^{2}\left\langle 2 \ell_{2}\right\rangle[35]^{4}}{\langle 61\rangle\left\langle 6 \ell_{1}\right\rangle\left\langle\ell_{1} \ell_{2}\right\rangle\left\langle\ell_{2} \ell_{1}\right\rangle\left\langle 2\left|P_{\ell_{1} \ell_{2} 2}\right| 5\right]\left\langle\ell_{1}\left|P_{\ell_{1} \ell_{2} 2}\right| 3\right][34][45]\left(P_{\ell_{1} \ell_{2} 2}^{2}\right)} \\
- & 2\left\langle 1 \ell_{1}\right\rangle^{2}\left\langle 1 \ell_{2}\right\rangle\left\langle 4 \ell_{1}\right\rangle^{2}\left\langle 4\left|P_{\ell_{2} 23}\right| 3\right]^{2}\left[\ell_{2} 3\right]^{2} \\
& \frac{\langle 45\rangle\left\langle 5 \ell_{1}\right\rangle\langle 61\rangle\left\langle 6 \ell_{1}\right\rangle\left\langle\ell_{2} \ell_{1}\right\rangle\left\langle 4\left|P_{\ell_{2} 23}\right| \ell_{2}\right]\left\langle\ell_{1}\left|P_{\ell_{2} 23}\right| 3\right][23]\left[\ell_{2} 2\right]\left(P_{\ell_{2} 23}^{2}\right)}{}
\end{aligned}
$$

In the following formulas, for this cut only, we define

$$
\left|\omega_{5}\right\rangle=P_{61} P_{23}|4\rangle
$$


and

$$
Q=\left(P_{23}^{2} / P_{61}^{2}\right) P_{61}+P_{23}
$$

\section{Rational contribution from $C_{61}$}

$$
C_{61}^{r a t}=C_{61}^{(r, 1)}+C_{61}^{(r, 2)}+C_{61}^{(r, 3)}+C_{61}^{(r, 4)}+C_{61}^{(r, 5)}
$$

The term $C_{61}^{(r, 1)}$

i) $t$-integrated formula:

$$
C_{61}^{(r, 1)}=\frac{2\langle\ell d \ell\rangle[\ell d \ell]\langle 1 \ell\rangle^{2}\langle 21\rangle\langle 2 \ell\rangle^{2}[1 \ell][35]^{4}\left(P_{61}^{2}\right)}{\langle 6 \ell\rangle^{2}\left\langle 2\left|P_{612}\right| 5\right]\left\langle\ell\left|P_{61}\right| \ell\right]^{3}\left\langle\ell\left|P_{612}\right| 3\right][34][45]\left(P_{612}^{2}\right)}
$$

We describe in detail how to write this term as a full derivative to show a technical point which allows us to get rid of higher poles - when possible.

We have seen in many examples that a suitable choice of the reference spinor $\eta$ can simplify writing an integrand as a full derivative, when using the integration-by-parts identity eq.(2.14). In the above expression there is a double pole $\langle 6 \ell\rangle^{2}$, but the presence in the numerator of $[1 \ell]$ seems to force us to pick up $\eta=1$. By doing so, one would end up with an expression containing a triple pole $\langle\ell 6\rangle^{3}$, to be dealt with afterwards.

Alternatively, one can multiply $C_{61}^{(r, 1)}$ by $1=[6 \ell] /[6 \ell]$ and use the following identity,

$$
\frac{[1 \ell]}{\left\langle\ell\left|P_{61}\right| \ell\right][6 \ell]}=\frac{1}{\left\langle\ell\left|P_{61}\right| 6\right]}\left(\frac{\left\langle\ell\left|P_{61}\right| 1\right]}{\left\langle\ell\left|P_{61}\right| \ell\right]}+\frac{[16]}{[6 \ell]}\right)
$$

to write $C_{61}^{(r, 1)}$ as a sum of two terms,

$$
\begin{aligned}
C_{61}^{(r, 1)} & =-\frac{2\langle\ell d \ell\rangle[\ell d \ell]\langle 1 \ell\rangle\langle 21\rangle\langle 2 \ell\rangle^{2}[35]^{4} P_{61}}{\langle 6 \ell\rangle^{2}\left\langle 2\left|P_{612}\right| 5\right]\left\langle\ell\left|P_{61}\right| \ell\right]^{2}\left\langle\ell\left|P_{612}\right| 3\right][34][45]\left(P_{612}^{2}\right)} \\
& +\frac{2\langle\ell d \ell\rangle[\ell d \ell]\langle 1 \ell\rangle\langle 21\rangle\langle 2 \ell\rangle^{2}[35]^{4}[61][6 \ell] P_{61}}{\langle 6 \ell\rangle\left\langle 2\left|P_{612}\right| 5\right]\left\langle\ell\left|P_{61}\right| \ell\right]^{3}\left\langle\ell\left|P_{612}\right| 3\right][16][34][45]\left(P_{612}^{2}\right)}
\end{aligned}
$$

each of which can be integrated by parts by using $\eta=6$, to neutralize the double pole. In fact its expression can be written as,

ii) full derivative:

$$
C_{61}^{(r, 1)}=\langle\ell d \ell\rangle\left[d \ell \partial_{\ell}\right] \mathcal{I}^{(1)}
$$




$$
\begin{aligned}
\mathcal{I}^{(1)} & =-\frac{2\langle 21\rangle\langle 2 \ell\rangle^{2}[35]^{4}[6 \ell]\left(P_{61}^{2}\right)}{\langle 6 \ell\rangle^{2}\left\langle 2\left|P_{612}\right| 5\right]\left\langle\ell\left|P_{61}\right| \ell\right]\left\langle\ell\left|P_{612}\right| 3\right][16][34][45]\left(P_{612}^{2}\right)} \\
& +\frac{\langle 21\rangle\langle 2 \ell\rangle^{2}[35]^{4}[61][6 \ell]^{2}\left(P_{61}^{2}\right)}{\langle 6 \ell\rangle\left\langle 2\left|P_{612}\right| 5\right]\left\langle\ell\left|P_{61}\right| \ell\right]^{2}\left\langle\ell\left|P_{612}\right| 3\right][16]^{2}[34][45]\left(P_{612}^{2}\right)}
\end{aligned}
$$

where the term with $1 /\langle 6 \ell\rangle^{2}$ has a factor in the numerator of $[6 \ell]$, which annihilates its residue. Therefore the contribution of this term to the corresponding bubble coefficients will be given by the sum of residues of only simple poles.

The term $C_{61}^{(r, 2)}$

i) $t$-integrated formula:

$$
\begin{aligned}
C_{61}^{(r, 2)} & =-\frac{2\langle\ell d \ell\rangle[\ell d \ell]\langle 1 \ell\rangle^{3}\langle 21\rangle\langle 2 \ell\rangle^{2}[16][1 \ell]^{2}[35]^{4}\left(P_{61}^{2}\right)}{\langle 6 \ell\rangle^{2}\left\langle 2\left|P_{612}\right| 5\right]\left\langle\ell\left|P_{61}\right| \ell\right]^{4}\left\langle\ell\left|P_{612}\right| 3\right][34][45][61]\left(P_{612}^{2}\right)} \\
& -\frac{2\langle\ell d \ell\rangle[\ell d \ell]\langle 1 \ell\rangle^{2}\langle 26\rangle\langle 2 \ell\rangle^{2}[35]^{4}[6 \ell]^{2}\left(P_{61}^{2}\right)}{\langle 6 \ell\rangle\left\langle 2\left|P_{612}\right| 5\right]\left\langle\ell\left|P_{61}\right| \ell\right]^{4}\left\langle\ell\left|P_{612}\right| 3\right][34][45]\left(P_{612}^{2}\right)}
\end{aligned}
$$

ii) full derivative:

$$
\begin{gathered}
C_{61}^{(r, 2)}=\langle\ell d \ell\rangle\left[d \ell \partial_{\ell}\right] \mathcal{I}^{(2)} \\
\mathcal{I}^{(2)}=\frac{2\langle 21\rangle\langle 2 \ell\rangle^{2}[35]^{4}[6 \ell]\left(P_{61}^{2}\right)}{\langle 6 \ell\rangle^{2}\left\langle 2\left|P_{612}\right| 5\right]\left\langle\ell\left|P_{61}\right| \ell\right]\left\langle\ell\left|P_{612}\right| 3\right][34][45][61]\left(P_{612}^{2}\right)} \\
-\frac{2\langle 21\rangle\langle 2 \ell\rangle^{2}[35]^{4}[6 \ell]^{2}\left(P_{61}^{2}\right)}{\langle 6 \ell\rangle\left\langle 2\left|P_{612}\right| 5\right]\left\langle\ell\left|P_{61}\right| \ell\right]^{2}\left\langle\ell\left|P_{612}\right| 3\right][16][34][45]\left(P_{612}^{2}\right)} \\
-\frac{2\langle 1 \ell\rangle\langle 26\rangle\langle 2 \ell\rangle^{2}[35]^{4}[6 \ell]^{3}\left(P_{61}^{2}\right)}{3\langle 6 \ell\rangle\left\langle 2\left|P_{612}\right| 5\right]\left\langle\ell\left|P_{61}\right| \ell\right]^{3}\left\langle\ell\left|P_{612}\right| 3\right][16][34][45]\left(P_{612}^{2}\right)} \\
+\frac{2\langle 21\rangle\langle 2 \ell\rangle^{2}[35]^{4}[61][6 \ell]^{3}\left(P_{61}^{2}\right)}{3\left\langle 2\left|P_{612}\right| 5\right]\left\langle\ell\left|P_{61}\right| \ell\right]^{3}\left\langle\ell\left|P_{612}\right| 3\right][16]^{2}[34][45]\left(P_{612}^{2}\right)}
\end{gathered}
$$

The term $C_{61}^{(r, 3)}$ 
i) $t$-integrated formula:

$$
\begin{aligned}
C_{61}^{(r, 3)}= & -\frac{2\langle\ell d \ell\rangle[\ell d \ell]\langle 1 \ell\rangle^{3}\langle 46\rangle^{2}\langle 4 \ell\rangle^{2}\langle\ell|Q| 6]^{2}\left\langle\ell\left|P_{61}\right| 3\right]^{4}[16]}{\langle 45\rangle\langle 5 \ell\rangle\langle 6 \ell\rangle\left\langle\omega_{5} \ell\right\rangle\left\langle\ell\left|P_{61} Q\right| \ell\right\rangle^{3}\left\langle\ell\left|P_{61}\right| 2\right]\left\langle\ell\left|P_{61}\right| \ell\right]^{2}\left\langle\ell\left|P_{612}\right| 3\right][23]} \\
& -\frac{2\langle\ell d \ell\rangle[\ell d \ell]\langle 1 \ell\rangle^{3}\langle 42\rangle^{2}\langle 4 \ell\rangle^{2}\left\langle\ell\left|P_{61}\right| 3\right]^{2}[16][23]}{\langle 45\rangle\langle 5 \ell\rangle\langle 6 \ell\rangle\left\langle\omega_{5} \ell\right\rangle\left\langle\ell\left|P_{61} Q\right| \ell\right\rangle\left\langle\ell\left|P_{61}\right| 2\right]\left\langle\ell\left|P_{61}\right| \ell\right]^{2}\left\langle\ell\left|P_{612}\right| 3\right]} \\
& -\frac{4\langle\ell d \ell\rangle[\ell d \ell]\langle 1 \ell\rangle^{3}\langle 41\rangle\langle 42\rangle\langle 4 \ell\rangle^{2}\langle\ell|Q| 1]\left\langle\ell\left|P_{61}\right| 3\right]^{3}[61]}{\langle 45\rangle\langle 5 \ell\rangle\langle 6 \ell\rangle\left\langle\omega_{5} \ell\right\rangle\left\langle\ell\left|P_{61} Q\right| \ell\right\rangle^{2}\left\langle\ell\left|P_{61}\right| 2\right]\left\langle\ell\left|P_{61}\right| \ell\right]^{2}\left\langle\ell\left|P_{612}\right| 3\right]} \\
& -\frac{4\langle\ell d \ell\rangle[\ell d \ell]\langle 1 \ell\rangle^{3}\langle 42\rangle\langle 46\rangle\langle 4 \ell\rangle^{2}\langle\ell|Q| 6]\left\langle\ell\left|P_{61}\right| 3\right]^{3}[61]}{\langle 45\rangle\langle 5 \ell\rangle\langle 6 \ell\rangle\left\langle\omega_{5} \ell\right\rangle\left\langle\ell\left|P_{61} Q\right| \ell\right\rangle^{2}\left\langle\ell\left|P_{61}\right| 2\right]\left\langle\ell\left|P_{61}\right| \ell\right]^{2}\left\langle\ell\left|P_{612}\right| 3\right]} \\
& -\frac{2\langle\ell d \ell\rangle[\ell d \ell]\langle 1 \ell\rangle^{2}\langle 41\rangle^{2}\langle 4 \ell\rangle^{2}\langle\ell|Q| 1]\langle\ell|Q| 6]\left\langle\ell\left|P_{61}\right|_{3}\right]^{4}[61]}{\langle 45\rangle\langle 5 \ell\rangle\left\langle\omega_{5} \ell\right\rangle\left\langle\ell\left|P_{61} Q\right| \ell\right\rangle^{3}\left\langle\ell\left|P_{61}\right| 2\right]\left\langle\ell\left|P_{61}\right| \ell\right]^{2}\left\langle\ell\left|P_{612}\right| 3\right][23]} \\
& -\frac{4\langle\ell d \ell\rangle[\ell d \ell]\langle 1 \ell\rangle^{2}\langle 41\rangle\langle 46\rangle\langle 4 \ell\rangle^{2}\langle\ell|Q| 6]^{2}\left\langle\ell\left|P_{61}\right| 3\right]^{4}[61]}{\langle 45\rangle\langle 5 \ell\rangle\left\langle\omega_{5} \ell\right\rangle\left\langle\ell\left|P_{61} Q\right| \ell\right\rangle^{3}\left\langle\ell\left|P_{61}\right| 2\right]\left\langle\ell\left|P_{61}\right| \ell\right]^{2}\left\langle\ell\left|P_{612}\right| 3\right][23]}
\end{aligned}
$$

ii) full derivative:

$$
C_{61}^{(r, 3)}=\langle\ell d \ell\rangle\left[d \ell \partial_{\ell}\right] \mathcal{I}^{(3)}
$$




$$
\begin{aligned}
& \mathcal{I}^{(3)}=-\frac{2\langle 1 \ell\rangle^{2}\langle 42\rangle^{2}\langle 4 \ell\rangle^{2}\left\langle\ell\left|P_{61}\right| 3\right]^{2}[23][6 \ell]}{\langle 45\rangle\langle 5 \ell\rangle\langle 6 \ell\rangle\left\langle\omega_{5} \ell\right\rangle\left\langle\ell\left|P_{61} Q\right| \ell\right\rangle\left\langle\ell\left|P_{61}\right| 2\right]\left\langle\ell\left|P_{61}\right| \ell\right]\left\langle\ell\left|P_{612}\right| 3\right]} \\
& +\frac{2\langle 1 \ell\rangle^{3}\langle 46\rangle^{2}\langle a b\rangle^{6}\left\langle\eta_{1} 4\right\rangle^{3}\langle\ell|Q| 6]^{2}\left\langle\ell\left|P_{61}\right| 3\right]^{4}[16]\left[\eta_{1} \ell\right]}{\langle 45\rangle\langle 4 \ell\rangle\langle 5 \ell\rangle\langle 6 \ell\rangle\left\langle\eta_{1} \ell\right\rangle^{3}\left\langle\eta_{1} \eta_{2}\right\rangle^{3}\left\langle\omega_{5} \ell\right\rangle\left\langle b\left|P_{61} Q\right| b\right\rangle^{3}\left\langle\ell\left|P_{61}\right| 2\right]\left\langle\ell\left|P_{61}\right| \ell\right]\left\langle\ell\left|P_{61}\right| \eta_{1}\right]\left\langle\ell\left|P_{612}\right| 3\right][23]} \\
& 6\langle 1 \ell\rangle^{3}\langle 46\rangle^{2}\left\langle 4 \eta_{2}\right\rangle\langle a b\rangle^{6}\left\langle\eta_{1} 4\right\rangle^{2}\langle\ell|Q| 6]^{2}\left\langle\ell\left|P_{61}\right| 3\right]^{4}[16]\left[\eta_{1} \ell\right]
\end{aligned}
$$

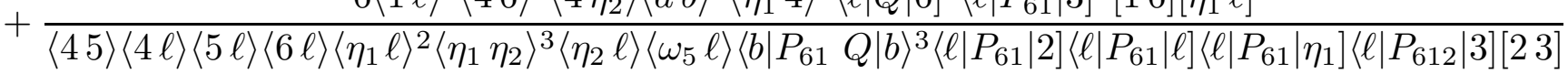

$$
\begin{aligned}
& 4\langle 1 \ell\rangle^{3}\langle 41\rangle\langle 42\rangle\langle a b\rangle^{4}\left\langle\eta_{1} 4\right\rangle^{2}\langle\ell|Q| 1]\left\langle\ell\left|P_{61}\right| 3\right]^{3}[61]\left[\eta_{1} \ell\right] \\
& \overline{\langle 45\rangle\langle 5 \ell\rangle\langle 6 \ell\rangle\left\langle\eta_{1} \ell\right\rangle^{2}\left\langle\eta_{1} \eta_{2}\right\rangle^{2}\left\langle\omega_{5} \ell\right\rangle\left\langle b\left|P_{61} Q\right| b\right\rangle^{2}\left\langle\ell\left|P_{61}\right| 2\right]\left\langle\ell\left|P_{61}\right| \ell\right]\left\langle\ell\left|P_{61}\right| \eta_{1}\right]\left\langle\ell\left|P_{612}\right| 3\right]} \\
& 8\langle 1 \ell\rangle^{3}\langle 41\rangle\langle 42\rangle\left\langle 4 \eta_{2}\right\rangle\langle a b\rangle^{4}\left\langle\eta_{1} 4\right\rangle\langle\ell|Q| 1]\left\langle\ell\left|P_{61}\right| 3\right]^{3}[61]\left[\eta_{1} \ell\right] \\
& +\frac{}{\langle 45\rangle\langle 5 \ell\rangle\langle 6 \ell\rangle\left\langle\eta_{1} \ell\right\rangle\left\langle\eta_{1} \eta_{2}\right\rangle^{2}\left\langle\eta_{2} \ell\right\rangle\left\langle\omega_{5} \ell\right\rangle\left\langle b\left|P_{61} Q\right| b\right\rangle^{2}\left\langle\ell\left|P_{61}\right| 2\right]\left\langle\ell\left|P_{61}\right| \ell\right]\left\langle\ell\left|P_{61}\right| \eta_{1}\right]\left\langle\ell\left|P_{612}\right| 3\right]} \\
& 4\langle 1 \ell\rangle^{3}\langle 42\rangle\langle 46\rangle\langle a b\rangle^{4}\left\langle\eta_{1} 4\right\rangle^{2}\langle\ell|Q| 6]\left\langle\ell\left|P_{61}\right| 3\right]^{3}[61]\left[\eta_{1} \ell\right] \\
& +\overline{\langle 45\rangle\langle 5 \ell\rangle\langle 6 \ell\rangle\left\langle\eta_{1} \ell\right\rangle^{2}\left\langle\eta_{1} \eta_{2}\right\rangle^{2}\left\langle\omega_{5} \ell\right\rangle\left\langle b\left|P_{61} Q\right| b\right\rangle^{2}\left\langle\ell\left|P_{61}\right| 2\right]\left\langle\ell\left|P_{61}\right| \ell\right]\left\langle\ell\left|P_{61}\right| \eta_{1}\right]\left\langle\ell\left|P_{612}\right| 3\right]} \\
& 8\langle 1 \ell\rangle^{3}\langle 42\rangle\langle 46\rangle\left\langle 4 \eta_{2}\right\rangle\langle a b\rangle^{4}\left\langle\eta_{1} 4\right\rangle\langle\ell|Q| 6]\left\langle\ell\left|P_{61}\right| 3\right]^{3}[61]\left[\eta_{1} \ell\right] \\
& +\frac{}{\langle 45\rangle\langle 5 \ell\rangle\langle 6 \ell\rangle\left\langle\eta_{1} \ell\right\rangle\left\langle\eta_{1} \eta_{2}\right\rangle^{2}\left\langle\eta_{2} \ell\right\rangle\left\langle\omega_{5} \ell\right\rangle\left\langle b\left|P_{61} Q\right| b\right\rangle^{2}\left\langle\ell\left|P_{61}\right| 2\right]\left\langle\ell\left|P_{61}\right| \ell\right]\left\langle\ell\left|P_{61}\right| \eta_{1}\right]\left\langle\ell\left|P_{612}\right| 3\right]} \\
& 2\langle 1 \ell\rangle^{2}\langle 41\rangle^{2}\langle a b\rangle^{6}\left\langle\eta_{1} 4\right\rangle^{3}\langle\ell|Q| 1]\langle\ell|Q| 6]\left\langle\ell\left|P_{61}\right| 3\right]^{4}[61]\left[\eta_{1} \ell\right] \\
& +\overline{\langle 45\rangle\langle 4 \ell\rangle\langle 5 \ell\rangle\left\langle\eta_{1} \ell\right\rangle^{3}\left\langle\eta_{1} \eta_{2}\right\rangle^{3}\left\langle\omega_{5} \ell\right\rangle\left\langle b\left|P_{61} Q\right| b\right\rangle^{3}\left\langle\ell\left|P_{61}\right| 2\right]\left\langle\ell\left|P_{61}\right| \ell\right]\left\langle\ell\left|P_{61}\right| \eta_{1}\right]\left\langle\ell\left|P_{612}\right| 3\right][23]} \\
& 6\langle 1 \ell\rangle^{2}\langle 41\rangle^{2}\left\langle 4 \eta_{2}\right\rangle\langle a b\rangle^{6}\left\langle\eta_{1} 4\right\rangle^{2}\langle\ell|Q| 1]\langle\ell|Q| 6]\left\langle\ell\left|P_{61}\right| 3\right]^{4}[61]\left[\eta_{1} \ell\right] \\
& +\frac{}{\langle 45\rangle\langle 4 \ell\rangle\langle 5 \ell\rangle\left\langle\eta_{1} \ell\right\rangle^{2}\left\langle\eta_{1} \eta_{2}\right\rangle^{3}\left\langle\eta_{2} \ell\right\rangle\left\langle\omega_{5} \ell\right\rangle\left\langle b\left|P_{61} Q\right| b\right\rangle^{3}\left\langle\ell\left|P_{61}\right| 2\right]\left\langle\ell\left|P_{61}\right| \ell\right]\left\langle\ell\left|P_{61}\right| \eta_{1}\right]\left\langle\ell\left|P_{612}\right| 3\right][23]} \\
& 4\langle 1 \ell\rangle^{2}\langle 41\rangle\langle 46\rangle\langle a b\rangle^{6}\left\langle\eta_{1} 4\right\rangle^{3}\langle\ell|Q| 6]^{2}\left\langle\ell\left|P_{61}\right| 3\right]^{4}[61]\left[\eta_{1} \ell\right] \\
& +\frac{155\rangle\langle 4 \ell\rangle\langle 5 \ell\rangle\left\langle\eta_{1} \ell\right\rangle^{3}\left\langle\eta_{1} \eta_{2}\right\rangle^{3}\left\langle\omega_{5} \ell\right\rangle\left\langle b\left|P_{61} Q\right| b\right\rangle^{3}\left\langle\ell\left|P_{61}\right| 2\right]\left\langle\ell\left|P_{61}\right| \ell\right]\left\langle\ell\left|P_{61}\right| \eta_{1}\right]\left\langle\ell\left|P_{612}\right| 3\right][23]}{\left.\langle 2\rangle^{2}\right]} \\
& 12\langle 1 \ell\rangle^{2}\langle 41\rangle\langle 46\rangle\left\langle 4 \eta_{2}\right\rangle\langle a b\rangle^{6}\left\langle\eta_{1} 4\right\rangle^{2}\langle\ell|Q| 6]^{2}\left\langle\ell\left|P_{61}\right| 3\right]^{4}[61]\left[\eta_{1} \ell\right] \\
& +\overline{\langle 45\rangle\langle 4 \ell\rangle\langle 5 \ell\rangle\left\langle\eta_{1} \ell\right\rangle^{2}\left\langle\eta_{1} \eta_{2}\right\rangle^{3}\left\langle\eta_{2} \ell\right\rangle\left\langle\omega_{5} \ell\right\rangle\left\langle b\left|P_{61} Q\right| b\right\rangle^{3}\left\langle\ell\left|P_{61}\right| 2\right]\left\langle\ell\left|P_{61}\right| \ell\right]\left\langle\ell\left|P_{61}\right| \eta_{1}\right]\left\langle\ell\left|P_{612}\right| 3\right][23]} \\
& 2\langle 1 \ell\rangle^{3}\langle 46\rangle^{2}\left\langle 4 \eta_{2}\right\rangle^{3}\langle a b\rangle^{6}\langle\ell|Q| 6]^{2}\left\langle\ell\left|P_{61}\right| 3\right]^{4}[16]\left[\eta_{2} \ell\right] \\
& +\frac{{ }^{2}}{\langle 45\rangle\langle 4 \ell\rangle\langle 5 \ell\rangle\langle 6 \ell\rangle\left\langle\eta_{1} \eta_{2}\right\rangle^{3}\left\langle\eta_{2} \ell\right\rangle^{3}\left\langle\omega_{5} \ell\right\rangle\left\langle b\left|P_{61} Q\right| b\right\rangle^{3}\left\langle\ell\left|P_{61}\right| 2\right]\left\langle\ell\left|P_{61}\right| \ell\right]\left\langle\ell\left|P_{61}\right| \eta_{2}\right]\left\langle\ell\left|P_{612}\right| 3\right][23]} \\
& 6\langle 1 \ell\rangle^{3}\langle 46\rangle^{2}\left\langle 4 \eta_{2}\right\rangle^{2}\langle a b\rangle^{6}\left\langle\eta_{1} 4\right\rangle\langle\ell|Q| 6]^{2}\left\langle\ell\left|P_{61}\right| 3\right]^{4}[16]\left[\eta_{2} \ell\right] \\
& +\frac{}{\langle 45\rangle\langle 4 \ell\rangle\langle 5 \ell\rangle\langle 6 \ell\rangle\left\langle\eta_{1} \ell\right\rangle\left\langle\eta_{1} \eta_{2}\right\rangle^{3}\left\langle\eta_{2} \ell\right\rangle^{2}\left\langle\omega_{5} \ell\right\rangle\left\langle b\left|P_{61} Q\right| b\right\rangle^{3}\left\langle\ell\left|P_{61}\right| 2\right]\left\langle\ell\left|P_{61}\right| \ell\right]\left\langle\ell\left|P_{61}\right| \eta_{2}\right]\left\langle\ell\left|P_{612}\right| 3\right][23]} \\
& 4\langle 1 \ell\rangle^{3}\langle 41\rangle\langle 42\rangle\left\langle 4 \eta_{2}\right\rangle^{2}\langle a b\rangle^{4}\langle\ell|Q| 1]\left\langle\ell\left|P_{61}\right| 3\right]^{3}[61]\left[\eta_{2} \ell\right]
\end{aligned}
$$

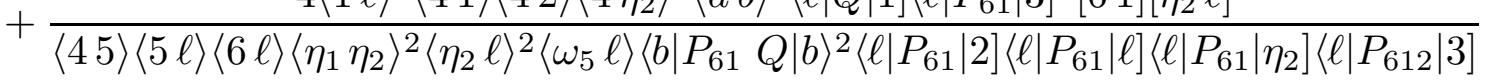

$$
\begin{aligned}
& 4\langle 1 \ell\rangle^{3}\langle 42\rangle\langle 46\rangle\left\langle 4 \eta_{2}\right\rangle^{2}\langle a b\rangle^{4}\langle\ell|Q| 6]\left\langle\ell\left|P_{61}\right| 3\right]^{3}[61]\left[\eta_{2} \ell\right]
\end{aligned}
$$

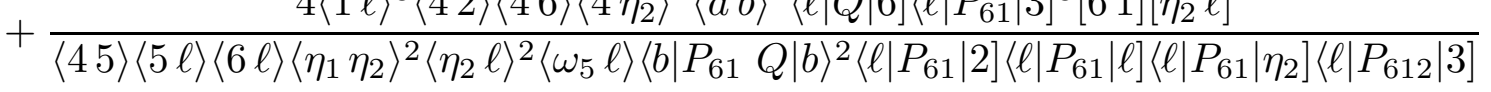

$$
\begin{aligned}
& 2\langle 1 \ell\rangle^{2}\langle 41\rangle^{2}\left\langle 4 \eta_{2}\right\rangle^{3}\langle a b\rangle^{6}\langle\ell|Q| 1]\langle\ell|Q| 6]\left\langle\ell\left|P_{61}\right| 3\right]^{4}[61]\left[\eta_{2} \ell\right] \\
& \overline{\langle 45\rangle\langle 4 \ell\rangle\langle 5 \ell\rangle\left\langle\eta_{1} \eta_{2}\right\rangle^{3}\left\langle\eta_{2} \ell\right\rangle^{3}\left\langle\omega_{5} \ell\right\rangle\left\langle b\left|P_{61} Q\right| b\right\rangle^{3}\left\langle\ell\left|P_{61}\right| 2\right]\left\langle\ell\left|P_{61}\right| \ell\right]\left\langle\ell\left|P_{61}\right| \eta_{2}\right]\left\langle\ell\left|P_{612}\right| 3\right][23]} \\
& 6\langle 1 \ell\rangle^{2}\langle 41\rangle^{2}\left\langle 4 \eta_{2}\right\rangle^{2}\langle a b\rangle^{6}\left\langle\eta_{1} 4\right\rangle\langle\ell|Q| 1]\langle\ell|Q| 6]\left\langle\ell\left|P_{61}\right| 3\right]^{4}[61]\left[\eta_{2} \ell\right] \\
& +\frac{{ }^{2}}{\langle 45\rangle\langle 4 \ell\rangle\langle 5 \ell\rangle\left\langle\eta_{1} \ell\right\rangle\left\langle\eta_{1} \eta_{2}\right\rangle^{3}\left\langle\eta_{2} \ell\right\rangle^{2}\left\langle\omega_{5} \ell\right\rangle\left\langle b\left|P_{61} Q\right| b\right\rangle^{3}\left\langle\ell\left|P_{61}\right| 2\right]\left\langle\ell\left|P_{61}\right| \ell\right]\left\langle\ell\left|P_{61}\right| \eta_{2}\right]\left\langle\ell\left|P_{612}\right| 3\right][23]} \\
& 4\langle 1 \ell\rangle^{2}\langle 41\rangle\langle 46\rangle\left\langle 4 \eta_{2}\right\rangle^{3}\langle a b\rangle^{6}\langle\ell|Q| 6]^{2}\left\langle\ell\left|P_{61}\right| 3\right]^{4}[61]\left[\eta_{2} \ell\right] \\
& +\frac{}{\langle 45\rangle\langle 4 \ell\rangle\langle 5 \ell\rangle\left\langle\eta_{1} \eta_{2}\right\rangle^{3}\left\langle\eta_{2} \ell\right\rangle^{3}\left\langle\omega_{5} \ell\right\rangle\left\langle b\left|P_{61} Q\right| b\right\rangle^{3}\left\langle\ell\left|P_{61}\right| 2\right]\left\langle\ell\left|P_{61}\right| \ell\right]\left\langle\ell\left|P_{61}\right| \eta_{2}\right]\left\langle\ell\left|P_{612}\right| 3\right][23]} \\
& 12\langle 1 \ell\rangle^{2}\langle 41\rangle\langle 46\rangle\left\langle 4 \eta_{2}\right\rangle^{2}\langle a b\rangle^{6}\left\langle\eta_{1} 4\right\rangle\langle\ell|Q| 6]^{2}\left\langle\ell\left|P_{61}\right| 3\right]^{4}[61]\left[\eta_{2} \ell\right] \\
& +\frac{}{\langle 45\rangle\langle 4 \ell\rangle\langle 5 \ell\rangle\left\langle\eta_{1} \ell\right\rangle\left\langle\eta_{1} \eta_{2}\right\rangle^{3}\left\langle\eta_{2} \ell\right\rangle^{2}\left\langle\omega_{5} \ell\right\rangle\left\langle b\left|P_{61} Q\right| b\right\rangle^{3}\left\langle\ell\left|P_{61}\right| 2\right]\left\langle\ell\left|P_{61}\right| \ell\right]\left\langle\ell\left|P_{61}\right| \eta_{2}\right]\left\langle\ell\left|P_{612}\right| 3\right][23]}
\end{aligned}
$$


The term $C_{61}^{(r, 4)}$

i) $t$-integrated formula:

$$
\begin{aligned}
C_{61}^{(r, 4)} & =\frac{4\langle\ell d \ell\rangle[\ell d \ell]\langle 1 \ell\rangle^{3}\langle 41\rangle\langle 46\rangle\langle 4 \ell\rangle^{2}\left\langle\ell\left|P_{61}\right| 3\right]^{4}[16][1 \ell]}{\langle 45\rangle\langle 5 \ell\rangle\langle 6 \ell\rangle^{2}\left\langle\omega_{5} \ell\right\rangle\left\langle\ell\left|P_{61} Q\right| \ell\right\rangle\left\langle\ell\left|P_{61}\right| 2\right]\left\langle\ell\left|P_{61}\right| \ell\right]^{3}\left\langle\ell\left|P_{612}\right| 3\right][23]} \\
+ & \frac{4\langle\ell d \ell\rangle[\ell d \ell]\langle 1 \ell\rangle^{3}\langle 41\rangle\langle 46\rangle\langle 4 \ell\rangle^{2}\langle\ell|Q| 6]\left\langle\ell\left|P_{61}\right| 3\right]^{4}[16][1 \ell]}{\langle 45\rangle\langle 5 \ell\rangle\langle 6 \ell\rangle\left\langle\omega_{5} \ell\right\rangle\left\langle\ell\left|P_{61} Q\right| \ell\right\rangle^{2}\left\langle\ell\left|P_{61}\right| 2\right]\left\langle\ell\left|P_{61}\right| \ell\right]^{3}\left\langle\ell\left|P_{612}\right| 3\right][23]} \\
+ & \frac{2\langle\ell d \ell\rangle[\ell d \ell]\langle 1 \ell\rangle^{2}\langle 41\rangle^{2}\langle 4 \ell\rangle^{2}\langle\ell|Q| 6]\left\langle\ell\left|P_{61}\right| 3\right]^{4}[1 \ell][61]}{\langle 45\rangle\langle 5 \ell\rangle\left\langle\omega_{5} \ell\right\rangle\left\langle\ell\left|P_{61} Q\right| \ell\right\rangle^{2}\left\langle\ell\left|P_{61}\right| 2\right]\left\langle\ell\left|P_{61}\right| \ell\right]^{3}\left\langle\ell\left|P_{612}\right| 3\right][23]} \\
+ & \frac{2\langle\ell d \ell\rangle[\ell d \ell]\langle 1 \ell\rangle^{3}\langle 46\rangle^{2}\langle 4 \ell\rangle^{2}\langle\ell|Q| 6]\left\langle\ell\left|P_{61}\right| 3\right]^{4}[16][6 \ell]}{\langle 45\rangle\langle 5 \ell\rangle\langle 6 \ell\rangle\left\langle\omega_{5} \ell\right\rangle\left\langle\ell\left|P_{61} Q\right| \ell\right\rangle^{2}\left\langle\ell\left|P_{61}\right| 2\right]\left\langle\ell\left|P_{61}\right| \ell\right]^{3}\left\langle\ell\left|P_{612}\right| 3\right][23]} \\
& -\frac{4\langle\ell d \ell\rangle[\ell d \ell]\langle 1 \ell\rangle^{2}\langle 41\rangle\langle 42\rangle\langle 4 \ell\rangle^{2}\left\langle\ell\left|P_{61}\right| 3\right]^{3}[61][6 \ell]}{\langle 45\rangle\langle 5 \ell\rangle\left\langle\omega_{5} \ell\right\rangle\left\langle\ell\left|P_{61} Q\right| \ell\right\rangle\left\langle\ell\left|P_{61}\right| 2\right]\left\langle\ell\left|P_{61}\right| \ell\right]^{3}\left\langle\ell\left|P_{612}\right| 3\right]} \\
+ & \frac{4\langle\ell d \ell\rangle[\ell d \ell]\langle 1 \ell\rangle^{3}\langle 42\rangle\langle 46\rangle\langle 4 \ell\rangle^{2}\left\langle\ell\left|P_{61}\right| 3\right]^{3}[61][6 \ell]}{\langle 45\rangle\langle 5 \ell\rangle\langle 6 \ell\rangle\left\langle\omega_{5} \ell\right\rangle\left\langle\ell\left|P_{61} Q\right| \ell\right\rangle\left\langle\ell\left|P_{61}\right| 2\right]\left\langle\ell\left|P_{61}\right| \ell\right]^{3}\left\langle\ell\left|P_{612}\right| 3\right]} \\
+ & \frac{2\langle\ell d \ell\rangle[\ell d \ell]\langle 1 \ell\rangle\langle 42\rangle^{4}\left\langle\ell\left|P_{61}\right| 5\right]^{2}[56][6 \ell]\left(P_{61}^{2}\right)}{\langle 23\rangle\langle 34\rangle\langle 6 \ell\rangle\left\langle\omega_{5} \ell\right\rangle\left\langle 2\left|P_{234}\right| 5\right]\left\langle\ell\left|P_{61}\right| \ell\right]^{3}[16]\left(P_{234}^{2}\right)}
\end{aligned}
$$

ii) full derivative:

$$
C_{61}^{(r, 4)}=\langle\ell d \ell\rangle\left[d \ell \partial_{\ell}\right] \mathcal{I}^{(4)}
$$




$$
\begin{aligned}
& \mathcal{I}^{(4)}=\frac{2\langle 1 \ell\rangle^{2}\langle 41\rangle^{2}\left\langle 4 \eta_{2}\right\rangle\langle a b\rangle^{4}\left\langle\eta_{1} 4\right\rangle\langle\ell|Q| 6]\left\langle\ell\left|P_{61}\right| 3\right]^{4}[1 \ell]^{2}}{\langle 45\rangle\langle 5 \ell\rangle\langle 6 \ell\rangle\left\langle\eta_{1} \ell\right\rangle\left\langle\eta_{1} \eta_{2}\right\rangle^{2}\left\langle\eta_{2} \ell\right\rangle\left\langle\omega_{5} \ell\right\rangle\left\langle b\left|P_{61} Q\right| b\right\rangle^{2}\left\langle\ell\left|P_{61}\right| 2\right]\left\langle\ell\left|P_{61}\right| \ell\right]^{2}\left\langle\ell\left|P_{612}\right| 3\right][23]} \\
& -\frac{4\langle 1 \ell\rangle\langle 41\rangle\langle 46\rangle\langle 4 \ell\rangle^{2}\langle a b\rangle^{2}\left\langle\ell\left|P_{61}\right| 3\right]^{4}[6 \ell]}{\langle 45\rangle\langle 5 \ell\rangle\langle 6 \ell\rangle^{2}\left\langle\eta_{1} \ell\right\rangle\left\langle\eta_{2} \ell\right\rangle\left\langle\omega_{5} \ell\right\rangle\left\langle b\left|P_{61} Q\right| b\right\rangle\left\langle\ell\left|P_{61}\right| 2\right]\left\langle\ell\left|P_{61}\right| \ell\right]\left\langle\ell\left|P_{612}\right| 3\right][23]} \\
& 8\langle 1 \ell\rangle\langle 41\rangle\langle 46\rangle\left\langle 4 \eta_{2}\right\rangle\langle a b\rangle^{4}\left\langle\eta_{1} 4\right\rangle\langle\ell|Q| 6]\left\langle\ell\left|P_{61}\right| 3\right]^{4}[6 \ell] \\
& -\frac{}{\langle 45\rangle\langle 5 \ell\rangle\langle 6 \ell\rangle\left\langle\eta_{1} \ell\right\rangle\left\langle\eta_{1} \eta_{2}\right\rangle^{2}\left\langle\eta_{2} \ell\right\rangle\left\langle\omega_{5} \ell\right\rangle\left\langle b\left|P_{61} Q\right| b\right\rangle^{2}\left\langle\ell\left|P_{61}\right| 2\right]\left\langle\ell\left|P_{61}\right| \ell\right]\left\langle\ell\left|P_{612}\right| 3\right][23]} \\
& +\frac{2\langle 1 \ell\rangle^{2}\langle 46\rangle^{2}\left\langle 4 \eta_{2}\right\rangle\langle a b\rangle^{4}\left\langle\eta_{1} 4\right\rangle\langle\ell|Q| 6]\left\langle\ell\left|P_{61}\right| 3\right]^{4}[6 \ell]^{2}}{\langle 45\rangle\langle 5 \ell\rangle\langle 6 \ell\rangle\left\langle\eta_{1} \ell\right\rangle\left\langle\eta_{1} \eta_{2}\right\rangle^{2}\left\langle\eta_{2} \ell\right\rangle\left\langle\omega_{5} \ell\right\rangle\left\langle b\left|P_{61} Q\right| b\right\rangle^{2}\left\langle\ell\left|P_{61}\right| 2\right]\left\langle\ell\left|P_{61}\right| \ell\right]^{2}\left\langle\ell\left|P_{612}\right| 3\right][23]} \\
& 2\langle 1 \ell\rangle\langle 41\rangle\langle 42\rangle\langle 4 \ell\rangle^{2}\langle a b\rangle^{2}\left\langle\ell\left|P_{61}\right| 3\right]^{3}[61][6 \ell]^{2} \\
& -\overline{\langle 45\rangle\langle 5 \ell\rangle\left\langle\eta_{1} \ell\right\rangle\left\langle\eta_{2} \ell\right\rangle\left\langle\omega_{5} \ell\right\rangle\left\langle b\left|P_{61} Q\right| b\right\rangle\left\langle\ell\left|P_{61}\right| 2\right]\left\langle\ell\left|P_{61}\right| \ell\right]^{2}\left\langle\ell\left|P_{612}\right| 3\right][16]} \\
& +\frac{2\langle 1 \ell\rangle^{2}\langle 42\rangle\langle 46\rangle\langle 4 \ell\rangle^{2}\langle a b\rangle^{2}\left\langle\ell\left|P_{61}\right| 3\right]^{3}[61][6 \ell]^{2}}{\langle 45\rangle\langle 5 \ell\rangle\langle 6 \ell\rangle\left\langle\eta_{1} \ell\right\rangle\left\langle\eta_{2} \ell\right\rangle\left\langle\omega_{5} \ell\right\rangle\left\langle b\left|P_{61} Q\right| b\right\rangle\left\langle\ell\left|P_{61}\right| 2\right]\left\langle\ell\left|P_{61}\right| \ell\right]^{2}\left\langle\ell\left|P_{612}\right| 3\right][16]} \\
& +\frac{2\langle 1 \ell\rangle\langle 41\rangle\langle 46\rangle\langle 4 \ell\rangle^{2}\langle a b\rangle^{2}\left\langle\ell\left|P_{61}\right| 3\right]^{4}[61][6 \ell]^{2}}{\langle 45\rangle\langle 5 \ell\rangle\langle 6 \ell\rangle\left\langle\eta_{1} \ell\right\rangle\left\langle\eta_{2} \ell\right\rangle\left\langle\omega_{5} \ell\right\rangle\left\langle b\left|P_{61} Q\right| b\right\rangle\left\langle\ell\left|P_{61}\right| 2\right]\left\langle\ell\left|P_{61}\right| \ell\right]^{2}\left\langle\ell\left|P_{612}\right| 3\right][16][23]} \\
& +\frac{4\langle 1 \ell\rangle\langle 41\rangle\langle 46\rangle\left\langle 4 \eta_{2}\right\rangle\langle a b\rangle^{4}\left\langle\eta_{1} 4\right\rangle\langle\ell|Q| 6]\left\langle\ell\left|P_{61}\right| 3\right]^{4}[61][6 \ell]^{2}}{\langle 45\rangle\langle 5 \ell\rangle\left\langle\eta_{1} \ell\right\rangle\left\langle\eta_{1} \eta_{2}\right\rangle^{2}\left\langle\eta_{2} \ell\right\rangle\left\langle\omega_{5} \ell\right\rangle\left\langle b\left|P_{61} Q\right| b\right\rangle^{2}\left\langle\ell\left|P_{61}\right| 2\right]\left\langle\ell\left|P_{61}\right| \ell\right]^{2}\left\langle\ell\left|P_{612}\right| 3\right][16][23]} \\
& -\frac{4\langle 1 \ell\rangle^{3}\langle 41\rangle\langle 46\rangle\langle a b\rangle^{4}\left\langle\eta_{1} 4\right\rangle^{2}\langle\ell|Q| 6]\left\langle\ell\left|P_{61}\right| 3\right]^{4}[16]\left[1 \eta_{1}\right]\left[\eta_{1} \ell\right]}{\langle 45\rangle\langle 5 \ell\rangle\langle 6 \ell\rangle\left\langle\eta_{1} \ell\right\rangle^{2}\left\langle\eta_{1} \eta_{2}\right\rangle^{2}\left\langle\omega_{5} \ell\right\rangle\left\langle b\left|P_{61} Q\right| b\right\rangle^{2}\left\langle\ell\left|P_{61}\right| 2\right]\left\langle\ell\left|P_{61}\right| \ell\right]\left\langle\ell\left|P_{61}\right| \eta_{1}\right]^{2}\left\langle\ell\left|P_{612}\right| 3\right][23]} \\
& 2\langle 1 \ell\rangle^{2}\langle 41\rangle^{2}\langle a b\rangle^{4}\left\langle\eta_{1} 4\right\rangle^{2}\langle\ell|Q| 6]\left\langle\ell\left|P_{61}\right| 3\right]^{4}\left[1 \eta_{1}\right][61]\left[\eta_{1} \ell\right] \\
& -\frac{}{\langle 45\rangle\langle 5 \ell\rangle\left\langle\eta_{1} \ell\right\rangle^{2}\left\langle\eta_{1} \eta_{2}\right\rangle^{2}\left\langle\omega_{5} \ell\right\rangle\left\langle b\left|P_{61} Q\right| b\right\rangle^{2}\left\langle\ell\left|P_{61}\right| 2\right]\left\langle\ell\left|P_{61}\right| \ell\right]\left\langle\ell\left|P_{61}\right| \eta_{1}\right]^{2}\left\langle\ell\left|P_{612}\right| 3\right][23]} \\
& 2\langle 1 \ell\rangle^{3}\langle 46\rangle^{2}\langle a b\rangle^{4}\left\langle\eta_{1} 4\right\rangle^{2}\langle\ell|Q| 6]\left\langle\ell\left|P_{61}\right| 3\right]^{4}[16]\left[6 \eta_{1}\right]\left[\eta_{1} \ell\right] \\
& -\overline{\langle 45\rangle\langle 5 \ell\rangle\langle 6 \ell\rangle\left\langle\eta_{1} \ell\right\rangle^{2}\left\langle\eta_{1} \eta_{2}\right\rangle^{2}\left\langle\omega_{5} \ell\right\rangle\left\langle b\left|P_{61} Q\right| b\right\rangle^{2}\left\langle\ell\left|P_{61}\right| 2\right]\left\langle\ell\left|P_{61}\right| \ell\right]\left\langle\ell\left|P_{61}\right| \eta_{1}\right]^{2}\left\langle\ell\left|P_{612}\right| 3\right][23]} \\
& +\frac{\langle 1 \ell\rangle^{4}\langle 46\rangle^{2}\langle a b\rangle^{4}\left\langle\eta_{1} 4\right\rangle^{2}\langle\ell|Q| 6]\left\langle\ell\left|P_{61}\right| 3\right]^{4}[16]^{2}\left[\eta_{1} \ell\right]^{2}}{\langle 45\rangle\langle 5 \ell\rangle\langle 6 \ell\rangle\left\langle\eta_{1} \ell\right\rangle^{2}\left\langle\eta_{1} \eta_{2}\right\rangle^{2}\left\langle\omega_{5} \ell\right\rangle\left\langle b\left|P_{61} Q\right| b\right\rangle^{2}\left\langle\ell\left|P_{61}\right| 2\right]\left\langle\ell\left|P_{61}\right| \ell\right]^{2}\left\langle\ell\left|P_{61}\right| \eta_{1}\right]^{2}\left\langle\ell\left|P_{612}\right| 3\right][23]} \\
& 2\langle 1 \ell\rangle^{3}\langle 41\rangle\langle 46\rangle\langle a b\rangle^{4}\left\langle\eta_{1} 4\right\rangle^{2}\langle\ell|Q| 6]\left\langle\ell\left|P_{61}\right| 3\right]^{4}[61]^{2}\left[\eta_{1} \ell\right]^{2} \\
& -\overline{\langle 45\rangle\langle 5 \ell\rangle\left\langle\eta_{1} \ell\right\rangle^{2}\left\langle\eta_{1} \eta_{2}\right\rangle^{2}\left\langle\omega_{5} \ell\right\rangle\left\langle b\left|P_{61} Q\right| b\right\rangle^{2}\left\langle\ell\left|P_{61}\right| 2\right]\left\langle\ell\left|P_{61}\right| \ell\right]^{2}\left\langle\ell\left|P_{61}\right| \eta_{1}\right]^{2}\left\langle\ell\left|P_{612}\right| 3\right][23]} \\
& +\frac{\langle 1 \ell\rangle^{2}\langle 41\rangle^{2}\langle 6 \ell\rangle\langle a b\rangle^{4}\left\langle\eta_{1} 4\right\rangle^{2}\langle\ell|Q| 6]\left\langle\ell\left|P_{61}\right| 3\right]^{4}[61]^{2}\left[\eta_{1} \ell\right]^{2}}{\langle 45\rangle\langle 5 \ell\rangle\left\langle\eta_{1} \ell\right\rangle^{2}\left\langle\eta_{1} \eta_{2}\right\rangle^{2}\left\langle\omega_{5} \ell\right\rangle\left\langle b\left|P_{61} Q\right| b\right\rangle^{2}\left\langle\ell\left|P_{61}\right| 2\right]\left\langle\ell\left|P_{61}\right| \ell\right]^{2}\left\langle\ell\left|P_{61}\right| \eta_{1}\right]^{2}\left\langle\ell\left|P_{612}\right| 3\right][23]} \\
& 4\langle 1 \ell\rangle^{3}\langle 41\rangle\langle 46\rangle\langle a b\rangle^{4}\left\langle\eta_{2} 4\right\rangle^{2}\langle\ell|Q| 6]\left\langle\ell\left|P_{61}\right| 3\right]^{4}[16]\left[1 \eta_{2}\right]\left[\eta_{2} \ell\right] \\
& -\frac{}{\langle 45\rangle\langle 5 \ell\rangle\langle 6 \ell\rangle\left\langle\eta_{2} \ell\right\rangle^{2}\left\langle\eta_{2} \eta_{1}\right\rangle^{2}\left\langle\omega_{5} \ell\right\rangle\left\langle b\left|P_{61} Q\right| b\right\rangle^{2}\left\langle\ell\left|P_{61}\right| 2\right]\left\langle\ell\left|P_{61}\right| \ell\right]\left\langle\ell\left|P_{61}\right| \eta_{2}\right]^{2}\left\langle\ell\left|P_{612}\right| 3\right][23]} \\
& 2\langle 1 \ell\rangle^{2}\langle 41\rangle^{2}\langle a b\rangle^{4}\left\langle\eta_{2} 4\right\rangle^{2}\langle\ell|Q| 6]\left\langle\ell\left|P_{61}\right| 3\right]^{4}\left[1 \eta_{2}\right][61]\left[\eta_{2} \ell\right] \\
& -\overline{\langle 45\rangle\langle 5 \ell\rangle\left\langle\eta_{2} \ell\right\rangle^{2}\left\langle\eta_{2} \eta_{1}\right\rangle^{2}\left\langle\omega_{5} \ell\right\rangle\left\langle b\left|P_{61} Q\right| b\right\rangle^{2}\left\langle\ell\left|P_{61}\right| 2\right]\left\langle\ell\left|P_{61}\right| \ell\right]\left\langle\ell\left|P_{61}\right| \eta_{2}\right]^{2}\left\langle\ell\left|P_{612}\right| 3\right][23]} \\
& 2\langle 1 \ell\rangle^{3}\langle 46\rangle^{2}\langle a b\rangle^{4}\left\langle\eta_{2} 4\right\rangle^{2}\langle\ell|Q| 6]\left\langle\ell\left|P_{61}\right| 3\right]^{4}[16]\left[6 \eta_{2}\right]\left[\eta_{2} \ell\right] \\
& \overline{\langle 45\rangle\langle 5 \ell\rangle\langle 6 \ell\rangle\left\langle\eta_{2} \ell\right\rangle^{2}\left\langle\eta_{2} \eta_{1}\right\rangle^{2}\left\langle\omega_{5} \ell\right\rangle\left\langle b\left|P_{61} Q\right| b\right\rangle^{2}\left\langle\ell\left|P_{61}\right| 2\right]\left\langle\ell\left|P_{61}\right| \ell\right]\left\langle\ell\left|P_{61}\right| \eta_{2}\right]^{2}\left\langle\ell\left|P_{612}\right| 3\right][23]}
\end{aligned}
$$$$
\text { (continues to the next page) }
$$ 
(continues from the previous page)

$$
\begin{aligned}
& +\frac{\langle 1 \ell\rangle^{4}\langle 46\rangle^{2}\langle a b\rangle^{4}\left\langle\eta_{2} 4\right\rangle^{2}\langle\ell|Q| 6]\left\langle\ell\left|P_{61}\right| 3\right]^{4}[16]^{2}\left[\eta_{2} \ell\right]^{2}}{\langle 45\rangle\langle 5 \ell\rangle\langle 6 \ell\rangle\left\langle\eta_{2} \ell\right\rangle^{2}\left\langle\eta_{2} \eta_{1}\right\rangle^{2}\left\langle\omega_{5} \ell\right\rangle\left\langle b\left|P_{61} Q\right| b\right\rangle^{2}\left\langle\ell\left|P_{61}\right| 2\right]\left\langle\ell\left|P_{61}\right| \ell\right]^{2}\left\langle\ell\left|P_{61}\right| \eta_{2}\right]^{2}\left\langle\ell\left|P_{612}\right| 3\right][23]} \\
& -\frac{2\langle 1 \ell\rangle^{3}\langle 41\rangle\langle 46\rangle\langle a b\rangle^{4}\left\langle\eta_{2} 4\right\rangle^{2}\langle\ell|Q| 6]\left\langle\ell\left|P_{61}\right| 3\right]^{4}[61]^{2}\left[\eta_{2} \ell\right]^{2}}{\langle 45\rangle\langle 5 \ell\rangle\left\langle\eta_{2} \ell\right\rangle^{2}\left\langle\eta_{2} \eta_{1}\right\rangle^{2}\left\langle\omega_{5} \ell\right\rangle\left\langle b\left|P_{61} Q\right| b\right\rangle^{2}\left\langle\ell\left|P_{61}\right| 2\right]\left\langle\ell\left|P_{61}\right| \ell\right]^{2}\left\langle\ell\left|P_{61}\right| \eta_{2}\right]^{2}\left\langle\ell\left|P_{612}\right| 3\right][23]} \\
& +\frac{\langle 1 \ell\rangle^{2}\langle 41\rangle^{2}\langle 6 \ell\rangle\langle a b\rangle^{4}\left\langle\eta_{2} 4\right\rangle^{2}\langle\ell|Q| 6]\left\langle\ell\left|P_{61}\right| 3\right]^{4}[61]^{2}\left[\eta_{2} \ell\right]^{2}}{\langle 45\rangle\langle 5 \ell\rangle\left\langle\eta_{2} \ell\right\rangle^{2}\left\langle\eta_{2} \eta_{1}\right\rangle^{2}\left\langle\omega_{5} \ell\right\rangle\left\langle b\left|P_{61} Q\right| b\right\rangle^{2}\left\langle\ell\left|P_{61}\right| 2\right]\left\langle\ell\left|P_{61}\right| \ell\right]^{2}\left\langle\ell\left|P_{61}\right| \eta_{2}\right]^{2}\left\langle\ell\left|P_{612}\right| 3\right][23]} \\
& +\frac{\langle 42\rangle^{4}\left\langle\ell\left|P_{61}\right| 5\right]^{2}[56][6 \ell]^{2}\left(P_{61}^{2}\right)}{\langle 23\rangle\langle 34\rangle\langle 6 \ell\rangle\left\langle\omega_{5} \ell\right\rangle\left\langle 2\left|P_{234}\right| 5\right]\left\langle\ell\left|P_{61}\right| \ell\right]^{2}[16]^{2}\left(P_{234}^{2}\right)}
\end{aligned}
$$

The above expression contains both single and double poles. Here we give only the expression for the residues of the double poles $\left\langle\ell\left|P_{61}\right| \eta_{1}\right]^{2}$ and $\left\langle\ell\left|P_{61}\right| \eta_{2}\right]^{2}$,

$$
C_{61}^{(r, 4: d)}=\langle\ell d \ell\rangle\left[d \ell \partial_{\ell}\right] \mathcal{I}^{(4: d)}
$$

$$
\begin{aligned}
\mathcal{I}^{(4: d)} & =-\frac{4\langle 1 \ell\rangle^{3}\langle 41\rangle\langle 46\rangle\langle a b\rangle^{4}\left\langle\eta_{1} 4\right\rangle^{2}\langle\ell|Q| 6]\left\langle\ell\left|P_{61}\right| 3\right]^{4}[16]\left[1 \eta_{1}\right]\left[\eta_{1} \ell\right]}{\langle 45\rangle\langle 5 \ell\rangle\langle 6 \ell\rangle\left\langle\eta_{1} \ell\right\rangle^{2}\left\langle\eta_{1} \eta_{2}\right\rangle^{2}\left\langle\omega_{5} \ell\right\rangle\left\langle b\left|P_{61} Q\right| b\right\rangle^{2}\left\langle\ell\left|P_{61}\right| 2\right]\left\langle\ell\left|P_{61}\right| \ell\right]\left\langle\ell\left|P_{61}\right| \eta_{1}\right]^{2}\left\langle\ell\left|P_{612}\right| 3\right][23]} \\
& -\frac{2\langle 1 \ell\rangle^{2}\langle 41\rangle^{2}\langle a b\rangle^{4}\left\langle\eta_{1} 4\right\rangle^{2}\langle\ell|Q| 6]\left\langle\ell\left|P_{61}\right| 3\right]^{4}\left[1 \eta_{1}\right][61]\left[\eta_{1} \ell\right]}{\langle 45\rangle\langle 5 \ell\rangle\left\langle\eta_{1} \ell\right\rangle^{2}\left\langle\eta_{1} \eta_{2}\right\rangle^{2}\left\langle\omega_{5} \ell\right\rangle\left\langle b\left|P_{61} Q\right| b\right\rangle^{2}\left\langle\ell\left|P_{61}\right| 2\right]\left\langle\ell\left|P_{61}\right| \ell\right]\left\langle\ell\left|P_{61}\right| \eta_{1}\right]^{2}\left\langle\ell\left|P_{612}\right| 3\right][23]} \\
& -\frac{2\langle 1 \ell\rangle^{3}\langle 46\rangle^{2}\langle a b\rangle^{4}\left\langle\eta_{1} 4\right\rangle^{2}\langle\ell|Q| 6]\left\langle\ell\left|P_{61}\right| 3\right]^{4}[16]\left[6 \eta_{1}\right]\left[\eta_{1} \ell\right]}{\langle 45\rangle\langle 5 \ell\rangle\langle 6 \ell\rangle\left\langle\eta_{1} \ell\right\rangle^{2}\left\langle\eta_{1} \eta_{2}\right\rangle^{2}\left\langle\omega_{5} \ell\right\rangle\left\langle b\left|P_{61} Q\right| b\right\rangle^{2}\left\langle\ell\left|P_{61}\right| 2\right]\left\langle\ell\left|P_{61}\right| \ell\right]\left\langle\ell\left|P_{61}\right| \eta_{1}\right]^{2}\left\langle\ell\left|P_{612}\right| 3\right][23]} \\
& +\frac{\langle 1 \ell\rangle^{4}\langle 46\rangle^{2}\langle a b\rangle^{4}\left\langle\eta_{1} 4\right\rangle^{2}\langle\ell|Q| 6]\left\langle\ell\left|P_{61}\right| 3\right]^{4}[16]^{2}\left[\eta_{1} \ell\right]^{2}}{\langle 45\rangle\langle 5 \ell\rangle\langle 6 \ell\rangle\left\langle\eta_{1} \ell\right\rangle^{2}\left\langle\eta_{1} \eta_{2}\right\rangle^{2}\left\langle\omega_{5} \ell\right\rangle\left\langle b\left|P_{61} Q\right| b\right\rangle^{2}\left\langle\ell\left|P_{61}\right| 2\right]\left\langle\ell\left|P_{61}\right| \ell\right]^{2}\left\langle\ell\left|P_{61}\right| \eta_{1}\right]^{2}\left\langle\ell\left|P_{612}\right| 3\right][23]} \\
& -\frac{2\langle 1 \ell\rangle^{3}\langle 41\rangle\langle 46\rangle\langle a b\rangle^{4}\left\langle\eta_{1} 4\right\rangle^{2}\langle\ell|Q| 6]\left\langle\ell\left|P_{61}\right| 3\right]^{4}[61]^{2}\left[\eta_{1} \ell\right]^{2}}{\langle 45\rangle\langle 5 \ell\rangle\left\langle\eta_{1} \ell\right\rangle^{2}\left\langle\eta_{1} \eta_{2}\right\rangle^{2}\left\langle\omega_{5} \ell\right\rangle\left\langle b\left|P_{61} Q\right| b\right\rangle^{2}\left\langle\ell\left|P_{61}\right| 2\right]\left\langle\ell\left|P_{61}\right| \ell\right]^{2}\left\langle\ell\left|P_{61}\right| \eta_{1}\right]^{2}\left\langle\ell\left|P_{612}\right| 3\right][23]} \\
& +\frac{\langle 1 \ell\rangle^{2}\langle 41\rangle^{2}\langle 6 \ell\rangle\langle a b\rangle^{4}\left\langle\eta_{1} 4\right\rangle^{2}\langle\ell|Q| 6]\left\langle\ell\left|P_{61}\right| 3\right]^{4}[61]^{2}\left[\eta_{1} \ell\right]^{2}}{\langle 45\rangle\langle 5 \ell\rangle\left\langle\eta_{1} \ell\right\rangle^{2}\left\langle\eta_{1} \eta_{2}\right\rangle^{2}\left\langle\omega_{5} \ell\right\rangle\left\langle b\left|P_{61} Q\right| b\right\rangle^{2}\left\langle\ell\left|P_{61}\right| 2\right]\left\langle\ell\left|P_{61}\right| \ell\right]^{2}\left\langle\ell\left|P_{61}\right| \eta_{1}\right]^{2}\left\langle\ell\left|P_{612}\right| 3\right][23]} \\
+ & \left\{\eta_{1} \leftrightarrow \eta_{2}\right\} \quad
\end{aligned}
$$


The sum of residue of the double poles reads,

$$
\begin{aligned}
& \left.C_{61}^{(r, 4: d)}=-\frac{4\langle 41\rangle\langle 46\rangle\langle a b\rangle^{4}\left\langle\eta_{1} 4\right\rangle^{2}[16]\left[1 \eta_{1}\right]\left\langle\eta_{1}\left|P_{61}\right| \eta_{1}\right]}{\langle 45\rangle\left\langle\eta_{1} \eta_{2}\right\rangle^{2}\left\langle b\left|P_{61} Q\right| b\right\rangle^{2}[23]\left(P_{61}^{2}\right)} P_{2}\left[P_{61} \mid \eta_{1}\right], L_{1}^{I I: C_{61}}, L_{2}^{I I: C_{61}}\right] \\
& \left.-\frac{2\langle 41\rangle^{2}\langle a b\rangle^{4}\left[1 \eta_{1}\right][61]\left\langle\eta_{1} 4\right\rangle^{2}\left\langle\eta_{1}\left|P_{61}\right| \eta_{1}\right]}{\langle 45\rangle\left\langle\eta_{1} \eta_{2}\right\rangle^{2}\left\langle b\left|P_{61} Q\right| b\right\rangle^{2}[23]\left(P_{61}^{2}\right)} P_{2}\left[P_{61} \mid \eta_{1}\right], M_{1}^{I I: C_{61}}, M_{2}^{I I: C_{61}}\right] \\
& \left.-\frac{2\langle 46\rangle^{2}\langle a b\rangle^{4}\left\langle\eta_{1} 4\right\rangle^{2}[16]\left[6 \eta_{1}\right]\left\langle\eta_{1}\left|P_{61}\right| \eta_{1}\right]}{\langle 45\rangle\left\langle\eta_{1} \eta_{2}\right\rangle^{2}\left\langle b\left|P_{61} Q\right| b\right\rangle^{2}[23]\left(P_{61}^{2}\right)} P_{2}\left[P_{61} \mid \eta_{1}\right], L_{1}^{I I: C_{61}}, L_{2}^{I I: C_{61}}\right] \\
& \left.-\frac{\langle 46\rangle^{2}\langle a b\rangle^{4}\left\langle\eta_{1} 4\right\rangle^{2}[16]^{2}\left\langle\eta_{1}\left|P_{61}\right| \eta_{1}\right]^{2}}{\langle 45\rangle\left\langle\eta_{1} \eta_{2}\right\rangle^{2}\left\langle b\left|P_{61} Q\right| b\right\rangle^{2}[23]\left(P_{61}^{2}\right)^{2}} P_{2}\left[P_{61} \mid \eta_{1}\right], N_{1}^{I I: C_{61}}, N_{2}^{I I: C_{61}}\right] \\
& \left.+\frac{2\langle 41\rangle\langle 46\rangle\langle a b\rangle^{4}\left\langle\eta_{1} 4\right\rangle^{2}[61]^{2}\left\langle\eta_{1}\left|P_{61}\right| \eta_{1}\right]^{2}}{\langle 45\rangle\left\langle\eta_{1} \eta_{2}\right\rangle^{2}\left\langle b\left|P_{61} Q\right| b\right\rangle^{2}[23]\left(P_{61}^{2}\right)^{2}} P_{2}\left[P_{61} \mid \eta_{1}\right], L_{1}^{I I: C_{61}}, O_{2}^{I I: C_{61}}\right] \\
& \left.-\frac{\langle 41\rangle^{2}\langle a b\rangle^{4}\left\langle\eta_{1} 4\right\rangle^{2}[61]^{2}\left\langle\eta_{1}\left|P_{61}\right| \eta_{1}\right]^{2}}{\langle 45\rangle\left\langle\eta_{1} \eta_{2}\right\rangle^{2}\left\langle b\left|P_{61} Q\right| b\right\rangle^{2}[23]\left(P_{61}^{2}\right)^{2}} P_{2}\left[P_{61} \mid \eta_{1}\right], O_{1}^{I I: C_{61}}, O_{2}^{I I: C_{61}}\right] \\
& +\left\{\eta_{1} \leftrightarrow \eta_{2}\right\}
\end{aligned}
$$

with

$$
\begin{aligned}
L_{1}^{I I: C_{61}} & \left.\left.\left.\left.\left.=\{|1\rangle,|1\rangle,|1\rangle, Q \mid 6], P_{61} \mid 3\right], P_{61} \mid 3\right], P_{61} \mid 3\right], P_{61} \mid 3\right]\right\} \\
L_{2}^{I I: C_{61}} & \left.\left.=\left\{|5\rangle,|6\rangle,\left|\eta_{1}\right\rangle,\left|\eta_{1}\right\rangle,\left|\eta_{1}\right\rangle,\left|\omega_{5}\right\rangle, P_{61} \mid 2\right], P_{612} \mid 3\right]\right\} \\
M_{1}^{I I: C_{61}} & \left.\left.\left.\left.\left.=\{|1\rangle,|1\rangle, Q \mid 6], P_{61} \mid 3\right], P_{61} \mid 3\right], P_{61} \mid 3\right], P_{61} \mid 3\right]\right\} \\
M_{2}^{I I: C_{61}} & \left.\left.=\left\{|5\rangle,\left|\eta_{1}\right\rangle,\left|\eta_{1}\right\rangle,\left|\eta_{1}\right\rangle,\left|\omega_{5}\right\rangle, P_{61} \mid 2\right], P_{612} \mid 3\right]\right\} \\
N_{1}^{I I: C_{61}} & \left.\left.\left.\left.\left.=\{|1\rangle,|1\rangle,|1\rangle,|1\rangle, Q \mid 6], P_{61} \mid 3\right], P_{61} \mid 3\right], P_{61} \mid 3\right], P_{61} \mid 3\right]\right\} \\
N_{2}^{I I: C_{61}} & \left.\left.=\left\{|5\rangle,|6\rangle,\left|\eta_{1}\right\rangle,\left|\eta_{1}\right\rangle,\left|\eta_{1}\right\rangle,\left|\eta_{1}\right\rangle,\left|\omega_{5}\right\rangle, P_{61} \mid 2\right], P_{612} \mid 3\right]\right\} \\
O_{1}^{I I: C_{61}} & \left.\left.\left.\left.\left.=\{|1\rangle,|1\rangle,|6\rangle, Q \mid 6], P_{61} \mid 3\right], P_{61} \mid 3\right], P_{61} \mid 3\right], P_{61} \mid 3\right]\right\} \\
O_{2}^{I I: C_{61}} & \left.\left.=\left\{|5\rangle,\left|\eta_{1}\right\rangle,\left|\eta_{1}\right\rangle,\left|\eta_{1}\right\rangle,\left|\eta_{1}\right\rangle,\left|\omega_{5}\right\rangle, P_{61} \mid 2\right], P_{612} \mid 3\right]\right\}
\end{aligned}
$$

since we used $\mid \ell]=P_{61}\left|\eta_{1}\right\rangle$.

The term $C_{61}^{(r, 5)}$

i) $t$-integrated formula:

$$
\begin{aligned}
C_{61}^{(r, 5)} & =-\frac{2\langle\ell d \ell\rangle[\ell d \ell]\langle 1 \ell\rangle^{3}\langle 41\rangle^{2}\langle 4 \ell\rangle^{2}\left\langle\ell\left|P_{61}\right| 3\right]^{4}[16][1 \ell]^{2}}{\langle 45\rangle\langle 5 \ell\rangle\langle 6 \ell\rangle\left\langle\omega_{5} \ell\right\rangle\left\langle\ell\left|P_{61} Q\right| \ell\right\rangle\left\langle\ell\left|P_{61}\right| 2\right]\left\langle\ell\left|P_{61}\right| \ell\right]^{4}\left\langle\ell\left|P_{612}\right| 3\right][23]} \\
& -\frac{4\langle\ell d \ell\rangle[\ell d \ell]\langle 1 \ell\rangle^{4}\langle 41\rangle\langle 46\rangle\langle 4 \ell\rangle^{2}\left\langle\ell\left|P_{61}\right| 3\right]^{4}[16]^{2}[1 \ell]^{2}}{\langle 45\rangle\langle 5 \ell\rangle\langle 6 \ell\rangle^{2}\left\langle\omega_{5} \ell\right\rangle\left\langle\ell\left|P_{61} Q\right| \ell\right\rangle\left\langle\ell\left|P_{61}\right| 2\right]\left\langle\ell\left|P_{61}\right| \ell\right]^{4}\left\langle\ell\left|P_{612}\right| 3\right][23][61]} \\
& -\frac{2\langle\ell d \ell\rangle[\ell d \ell]\langle 1 \ell\rangle^{3}\langle 46\rangle^{2}\langle 4 \ell\rangle^{2}\left\langle\ell\left|P_{61}\right| 3\right]^{4}[16][6 \ell]^{2}}{\langle 45\rangle\langle 5 \ell\rangle\langle 6 \ell\rangle\left\langle\omega_{5} \ell\right\rangle\left\langle\ell\left|P_{61} Q\right| \ell\right\rangle\left\langle\ell\left|P_{61}\right| 2\right]\left\langle\ell\left|P_{61}\right| \ell\right]^{4}\left\langle\ell\left|P_{612}\right| 3\right][23]} \\
& +\frac{2\langle\ell d \ell\rangle[\ell d \ell]\langle 1 \ell\rangle\langle 42\rangle^{4}\left\langle\ell\left|P_{61}\right| 5\right]^{3}[6 \ell]^{2}\left(P_{61}^{2}\right)}{\langle 23\rangle\langle 34\rangle\langle 6 \ell\rangle\left\langle\omega_{5} \ell\right\rangle\left\langle 2\left|P_{234}\right| 5\right]\left\langle\ell\left|P_{61}\right| \ell\right]^{4}[16]\left(P_{234}^{2}\right)}
\end{aligned}
$$


ii) full derivative:

$$
\begin{gathered}
C_{61}^{(r, 5)}=\langle\ell d \ell\rangle\left[d \ell \partial_{\ell}\right] \mathcal{I}^{(5)} \\
\mathcal{I}^{(5)}=-\frac{2\langle 41\rangle^{2}\langle 4 \ell\rangle^{2}\left\langle\ell\left|P_{61}\right| 3\right]^{4}[6 \ell]}{\langle 45\rangle\langle 5 \ell\rangle\langle 6 \ell\rangle\left\langle\omega_{5} \ell\right\rangle\left\langle\ell\left|P_{61} Q\right| \ell\right\rangle\left\langle\ell\left|P_{61}\right| 2\right]\left\langle\ell\left|P_{61}\right| \ell\right]\left\langle\ell\left|P_{612}\right| 3\right][23]} \\
+\frac{2\langle 41\rangle^{2}\langle 4 \ell\rangle^{2}\left\langle\ell\left|P_{61}\right| 3\right]^{4}[61][6 \ell]^{2}}{\langle 45\rangle\langle 5 \ell\rangle\left\langle\omega_{5} \ell\right\rangle\left\langle\ell\left|P_{61} Q\right| \ell\right\rangle\left\langle\ell\left|P_{61}\right| 2\right]\left\langle\ell\left|P_{61}\right| \ell\right]^{2}\left\langle\ell\left|P_{612}\right| 3\right][16][23]} \\
-\frac{2\langle 1 \ell\rangle^{2}\langle 46\rangle^{2}\langle 4 \ell\rangle^{2}\left\langle\ell\left|P_{61}\right| 3\right]^{4}[6 \ell]^{3}}{3\langle 45\rangle\langle 5 \ell\rangle\langle 6 \ell\rangle\left\langle\omega_{5} \ell\right\rangle\left\langle\ell\left|P_{61} Q\right| \ell\right\rangle\left\langle\ell\left|P_{61}\right| 2\right]\left\langle\ell\left|P_{61}\right| \ell\right]^{3}\left\langle\ell\left|P_{612}\right| 3\right][23]} \\
-\frac{2\langle 41\rangle^{2}\langle 4 \ell\rangle^{2}\langle 6 \ell\rangle\left\langle\ell\left|P_{61}\right| 3\right]^{4}[61]^{2}[6 \ell]^{3}}{3\langle 45\rangle\langle 5 \ell\rangle\left\langle\omega_{5} \ell\right\rangle\left\langle\ell\left|P_{61} Q\right| \ell\right\rangle\left\langle\ell\left|P_{61}\right| 2\right]\left\langle\ell\left|P_{61}\right| \ell\right]^{3}\left\langle\ell\left|P_{612}\right| 3\right][16]^{2}[23]} \\
+\frac{2\langle 42\rangle^{4}\left\langle\ell\left|P_{61}\right| 5\right]^{3}[6 \ell]^{3}\left(P_{61}^{2}\right)}{3\langle 23\rangle\langle 34\rangle\langle 6 \ell\rangle\left\langle\omega_{5} \ell\right\rangle\left\langle 2\left|P_{234}\right| 5\right]\left\langle\ell\left|P_{61}\right| \ell\right]^{3}[16]^{2}\left(P_{234}^{2}\right)}
\end{gathered}
$$

Finally, the coefficient of the bubble $I_{2: 2 ; 6}$ can be written as a sum of the residues of all the poles, by adding eqs. (5.87), (5.90), (5.93), (5.96), (5.104), and (5.100):

$$
c_{2: 2 ; 6}=\sum_{j=1}^{10} \lim _{\ell \rightarrow \ell_{j}}\left\langle\ell \ell_{j}\right\rangle \sum_{i=1}^{5} \mathcal{I}^{(i)}+C_{61}^{(4: d)},
$$

where the single poles are

$$
\left.\left.\left.\left.\left|\ell_{j}\right\rangle=|4\rangle,|5\rangle,|6\rangle, P_{612} \mid 3\right],\left|\omega_{5}\right\rangle, P_{61} \mid 2\right],\left|\eta_{1}\right\rangle,\left|\eta_{2}\right\rangle, P_{61} \mid \eta_{1}\right], P_{61} \mid \eta_{2}\right], \quad(j=1, \ldots, 10)
$$

\section{3-Mass-Triangle contribution from $C_{61}$}

$$
C_{61}^{(3 m)}=C_{61}^{(3 m, 1)}+C_{61}^{(3 m, 2)}+C_{61}^{(3 m, 3)}
$$

The term $C_{61}^{(3 m, 1)}$

i) after $t$-integration:

$$
C_{61}^{(3 m, 1)}=\frac{2\langle 42\rangle^{2}[23]}{\langle 45\rangle} \frac{\langle\ell d \ell\rangle[\ell d \ell]\langle\ell 1\rangle^{2}\langle\ell 4\rangle^{2}\langle\ell|Q| 6]\left\langle\ell\left|P_{61}\right| 3\right]^{2}}{\langle\ell|Q| \ell]\left\langle\ell\left|P_{61}\right| \ell\right]\left\langle\ell\left|P_{61} Q\right| \ell\right\rangle\langle\ell 5\rangle\langle\ell 6\rangle\left\langle\ell \omega_{5}\right\rangle\left\langle\ell\left|P_{61}\right| 2\right]\left\langle\ell\left|P_{612}\right| 3\right]}
$$

ii) triangle coefficient:

$$
C_{61}^{(3 m, 1)}=\frac{2\langle 42\rangle^{2}[23]}{\langle 45\rangle} C_{3}^{I I}\left[L_{a}, L_{b}^{I I}, P_{61}, Q\right]
$$




$$
\begin{aligned}
L_{a} & \left.\left.\left.=\{|1\rangle,|1\rangle,|4\rangle,|4\rangle, Q \mid 6], P_{61} \mid 3\right], P_{61} \mid 3\right]\right\} \\
L_{b}^{I I} & \left.\left.=\left\{|5\rangle,|6\rangle,\left|\omega_{5}\right\rangle, P_{61} \mid 2\right], P_{612} \mid 3\right],|\eta\rangle\right\}
\end{aligned}
$$

The term $C_{61}^{(3 m, 2)}$

i) after $t$-integration:

$$
\begin{aligned}
C_{61}^{(3 m, 2)}= & -\frac{4\langle 41\rangle\langle 42\rangle}{\langle 45\rangle} \frac{\langle\ell d \ell\rangle[\ell d \ell]\langle\ell 1\rangle^{2}\langle\ell 4\rangle^{2}\langle\ell|Q| 1]\langle\ell|Q| 6]\left\langle\ell\left|P_{61}\right| 3\right]^{3}}{\langle\ell|Q| \ell]\left\langle\ell\left|P_{61}\right| \ell\right]\left\langle\ell\left|P_{61} Q\right| \ell\right\rangle^{2}\langle\ell 5\rangle\langle\ell 6\rangle\left\langle\ell \omega_{5}\right\rangle\left\langle\ell\left|P_{61}\right| 2\right]\left\langle\ell\left|P_{612}\right| 3\right]} \\
& -\frac{4\langle 42\rangle\langle 46\rangle}{\langle 45\rangle} \frac{\langle\ell d \ell\rangle[\ell d \ell]\langle\ell 1\rangle^{2}\langle\ell 4\rangle^{2}\langle\ell|Q| 6]^{2}\left\langle\ell\left|P_{61}\right| 3\right]^{3}}{\langle\ell|Q| \ell]\left\langle\ell\left|P_{61}\right| \ell\right]\left\langle\ell\left|P_{61} Q\right| \ell\right\rangle^{2}\langle\ell 5\rangle\langle\ell 6\rangle\left\langle\ell \omega_{5}\right\rangle\left\langle\ell\left|P_{61}\right| 2\right]\left\langle\ell\left|P_{612}\right| 3\right]}
\end{aligned}
$$

ii) triangle coefficient:

$$
\begin{aligned}
& C_{61}^{(3 m, 2)}=-\frac{4\langle 41\rangle\langle 42\rangle}{\langle 45\rangle} C_{3}^{I I I}\left[L_{a}, L_{b, 1}^{I I I}, L_{b, 2}^{I I I}, P_{61}, Q\right] \\
&-\frac{4\langle 42\rangle\langle 46\rangle}{\langle 45\rangle} C_{3}^{I I I}\left[M_{a}, L_{b, 1}^{I I I}, L_{b, 2}^{I I I}, P_{61}, Q\right] \\
&\left.\left.\left.\left.\left.L_{a}=\{|1\rangle,|1\rangle,|4\rangle,|4\rangle, Q \mid 1], Q \mid 6\right], P_{61} \mid 3\right], P_{61} \mid 3\right], P_{61} \mid 3\right]\right\} \\
&\left.\left.\left.\left.\left.M_{a}=\{|1\rangle,|1\rangle,|4\rangle,|4\rangle, Q \mid 6], Q \mid 6\right], P_{61} \mid 3\right], P_{61} \mid 3\right], P_{61} \mid 3\right]\right\} \\
&\left.\left.L_{b, 1}^{I I I}=\left\{|5\rangle,|6\rangle,\left|\omega_{5}\right\rangle, P_{61} \mid 2\right], P_{612} \mid 3\right],|\eta\rangle,\left|\eta_{2}\right\rangle,\left|\eta_{2}\right\rangle\right\} \\
&\left.\left.L_{b, 2}^{I I I}=\left\{|5\rangle,|6\rangle,\left|\omega_{5}\right\rangle, P_{61} \mid 2\right], P_{612} \mid 3\right],|\eta\rangle,\left|\eta_{1}\right\rangle,\left|\eta_{1}\right\rangle\right\}
\end{aligned}
$$

The term $C_{61}^{(3 m, 3)}$

i) after $t$-integration:

$$
\begin{aligned}
C_{61}^{(3 m, 3)}= & \frac{2\langle 41\rangle^{2}}{\langle 45\rangle[23]} \frac{\langle\ell d \ell\rangle[\ell d \ell]\langle\ell 1\rangle^{2}\langle\ell 4\rangle^{2}\langle\ell|Q| 1]^{2}\langle\ell|Q| 6]\left\langle\ell\left|P_{61}\right| 3\right]^{4}}{\langle\ell| \ell]\left\langle\ell\left|P_{61}\right| \ell\right]\left\langle\ell\left|P_{61} Q\right| \ell\right\rangle^{3}\langle\ell 5\rangle\langle\ell 6\rangle\left\langle\ell \omega_{5}\right\rangle\left\langle\ell\left|P_{61}\right| 2\right]\left\langle\ell\left|P_{612}\right| 3\right]} \\
& +\frac{4\langle 41\rangle\langle 46\rangle}{\langle 45\rangle[23]} \frac{\langle\ell d \ell\rangle[\ell d \ell]\langle\ell 1\rangle^{2}\langle\ell 4\rangle^{2}\langle\ell|Q| 1]\langle\ell|Q| 6]^{2}\left\langle\ell\left|P_{61}\right| 3\right]^{4}}{\langle\ell|Q| \ell]\left\langle\ell\left|P_{61}\right| \ell\right]\left\langle\ell\left|P_{61} Q\right| \ell\right\rangle^{3}\langle\ell 5\rangle\langle\ell 6\rangle\left\langle\ell \omega_{5}\right\rangle\left\langle\ell\left|P_{61}\right| 2\right]\left\langle\ell\left|P_{612}\right| 3\right]} \\
& +\frac{2\langle 46\rangle^{2}}{\langle 45\rangle[23]} \frac{\langle\ell d \ell\rangle[\ell d \ell]\langle\ell 1\rangle^{2}\langle\ell 4\rangle^{2}\langle\ell|Q| 6]^{3}\left\langle\ell\left|P_{61}\right| 3\right]^{4}}{\langle\ell|Q| \ell]\left\langle\ell\left|P_{61}\right| \ell\right]\left\langle\ell\left|P_{61} Q\right| \ell\right\rangle^{3}\langle\ell 5\rangle\langle\ell 6\rangle\left\langle\ell \omega_{5}\right\rangle\left\langle\ell\left|P_{61}\right| 2\right]\left\langle\ell\left|P_{612}\right| 3\right]}
\end{aligned}
$$

ii) triangle coefficient:

$$
\begin{aligned}
C_{61}^{(3 m, 3)}= & \frac{2\langle 41\rangle^{2}}{\langle 45\rangle[23]} C_{3}^{I V}\left[L_{a}, L_{b, 1}^{I V}, L_{b, 2}^{I V}, P_{61}, Q\right] \\
& +\frac{4\langle 41\rangle\langle 46\rangle}{\langle 45\rangle[23]} C_{3}^{I V}\left[M_{a}, L_{b, 1}^{I V}, L_{b, 2}^{I V}, P_{61}, Q\right] \\
& +\frac{2\langle 46\rangle^{2}}{\langle 45\rangle[23]} C_{3}^{I V}\left[N_{a}, L_{b, 1}^{I V}, L_{b, 2}^{I V}, P_{61}, Q\right]
\end{aligned}
$$




$$
\begin{aligned}
L_{a} & \left.\left.\left.\left.\left.=\{|1\rangle,|1\rangle,|4\rangle,|4\rangle, Q \mid 1], Q \mid 1], Q \mid 6], P_{61} \mid 3\right], P_{61} \mid 3\right], P_{61} \mid 3\right], P_{61} \mid 3\right]\right\} \\
M_{a} & \left.\left.\left.\left.\left.=\{|1\rangle,|1\rangle,|4\rangle,|4\rangle, Q \mid 1], Q \mid 6], Q \mid 6], P_{61} \mid 3\right], P_{61} \mid 3\right], P_{61} \mid 3\right], P_{61} \mid 3\right]\right\} \\
N_{a} & \left.\left.\left.\left.\left.=\{|1\rangle,|1\rangle,|4\rangle,|4\rangle, Q \mid 6], Q \mid 6], Q \mid 6], P_{61} \mid 3\right], P_{61} \mid 3\right], P_{61} \mid 3\right], P_{61} \mid 3\right]\right\} \\
L_{b, 1}^{I V} & \left.\left.=\left\{|5\rangle,|6\rangle,\left|\omega_{5}\right\rangle, P_{61} \mid 2\right], P_{612} \mid 3\right],|\eta\rangle,\left|\eta_{2}\right\rangle,\left|\eta_{2}\right\rangle,\left|\eta_{2}\right\rangle\right\} \\
L_{b, 2}^{I V} & \left.\left.=\left\{|5\rangle,|6\rangle,\left|\omega_{5}\right\rangle, P_{61} \mid 2\right], P_{612} \mid 3\right],|\eta\rangle,\left|\eta_{1}\right\rangle,\left|\eta_{1}\right\rangle,\left|\eta_{1}\right\rangle\right\}
\end{aligned}
$$

Finally, the coefficient of the three-mass triangle $I_{3: 2: 2 ; 2}$ is given by the sum of (5.109), (5.112), and (5.115):

$$
c_{3: 2: 2 ; 2}=C_{61}^{(1,3 m, 1)}+C_{61}^{(1,3 m, 2)}+C_{61}^{(1,3 m, 3)} .
$$

\section{Conclusions}

In this work we completed the cut-constructible part of $\mathcal{A}^{\text {scalar }}$ for the one-loop sixgluon amplitude in QCD. This completes the calculation of the cut-constructible component of the one-loop six-gluon amplitude in QCD as whole.

The method we adopted from [12] relies on the combination of ideas belonging to generalized unitarity, to build the cuts out of tree-level amplitudes, and complex spinor algebra, to carry on the phase space integration. This method was already successfully

applied in the computation of the $\mathcal{A}^{\mathcal{N}=1}$ partner of the six-gluon amplitude. For the current task, we extended its features nontrivially to deal with the more general features of amplitudes belonging to less supersymmetric theories, such as QCD.

The background knowledge of two properties of one-loop amplitudes, namely supersymmetric decomposition, and integral reduction to a linear combination of analytically known scalar functions, associated to two-, three-, and four-point topologies, allowed us to concentrate mainly on the phase space integration.

By exploiting the finiteness of the amplitude, we chose an integral basis involving only boxes, three-mass triangles, and bubbles. Therefore, the problem of computing the (cutconstructible part of the) amplitude was shifted to the calculation of the corresponding coefficients - or rather to their extraction from the cut integrals.

Our goal was to reduce the complexity of the calculation as much as possible to trivial spinor algebra manipulations, and to minimize the number of actual integrations to be finally performed. 
The coefficients of the box functions could be computed via the quadruple-cut method without any integration whatsoever. Indeed, these have all appeared previously in the literature.

The known analytic properties of the bubble and three-mass triangle functions enabled us to distinguish unequivocally among them. To be more explicit, their branch cuts represented a specific signature to identify the coefficients of bubbles and three-mass triangles separately. The former multiply a term that after the integration would have generated a rational term; the latter multiply a term that after the integration would have generated logarithms with square roots in the arguments.

After this a priori analysis on the properties of the expected results, we could apply our optimized algorithm for the phase space integration. We wrote the 'twistor-motivated' Lorentz invariant phase space measure and the cut integrands in spinorial formalism, with the loop momentum written in its two components of opposite helicity, namely holomorphic and an antiholomorphic spinor variables. We used trivial spinor algebra to disentangle the dependence on the two variables and to write the integrands as a spinor derivative with respect to one of the two integration variables. We found that the expression of the result, at that stage of the calculation, contained only four classes of integrands.

The integration was finally performed by combining the holomorphic anomaly, which is an adaptation of the Cauchy residue theorem, and Feynman parametrization. That required the development of a technique for dealing with spinorial integrands carrying multiple poles, which constitutes the novel and the most powerful feature of our algorithm.

In view of the recent progress in understanding the recursive behaviour of scattering amplitudes, the results here obtained can, in principle, be used to drive the recursion relations for constructing the left-over rational piece of the six-gluon amplitude [13]. Moreover, due to its polylogarithmic structure, the amplitude we computed could represent a bootstrap point for the calculation of one-loop amplitudes with more external legs and different helicity configuration, once the one-loop recursive behaviour is fully sorted out.

The numerical implementation of the results here presented, and their crosschecks, is left to future work.

The method we have developed may be used to compute the cut-constructible part of a generic one-loop $n$-point amplitude with different particle content. 


\section{Acknowledgments}

We thank all the participants and organizers of the workshop "From Twistors To Amplitudes", and especially Babis Anastasiou and Nigel Glover, for the fruitful environment out of which this project started.

We would like to thank Freddy Cachazo and Thomas Gehrmann for feedback on the manuscript and Evgeny Buchbinder for conversations. P. M. wishes to thank Daniel Maitre for discussing useful features of Mathematica and Ettore Remiddi for an enjoyable discussion on complex analysis.

R. B. is supported by Stichting FOM. B. F. is supported by the Marie Curie Research Training Network under contract number MRTN-CT-2004-005104. P. M. has been partially supported by the US DOE grant DE-FG03-91ER40662 Task J, and by the European Commission Marie Curie Fellowship under contract number MEIF-CT-2006-024178.

\section{Appendix A. Tree-Level Amplitudes}

MHV tree amplitudes with fermions and scalars may be derived from supersymmetric Ward identities [63,34] applied to the Parke-Taylor formula [33]. See [23] for a review.

Here we summarize some NMHV tree-level amplitudes which are useful for our calculations. A similar list was given in Appendix B of [12]. However there is a technical point in these results, so we recall them again.

Each unitarity cut integral has two terms, from the two possible helicity assignments for the internal propagators. It is found that to simplify results, we would like the expressions for the tree amplitudes in each of these two terms to be related in a simple, symmetric way. For example, if we derive them by on-shell recursion relations [35, 36, 64, 65], we should take the same reference momenta for each pair at every step. Under this convention we have following results where $a=2$ is for scalars and $a=1$ is for fermions. Taking $a=0$ reproduces the results for all-gluon amplitudes; these may be used to check the relation $(2.3)$.

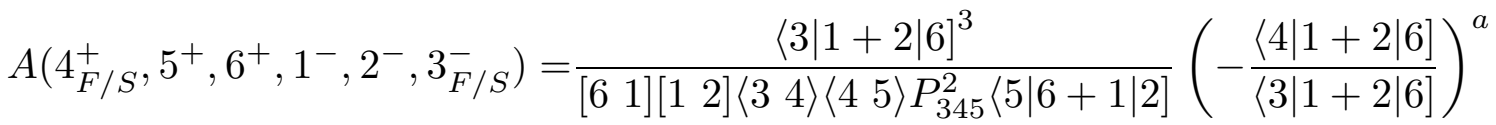

$$
\begin{aligned}
& +\frac{\langle 1|5+6| 4]^{3}}{\left[\begin{array}{lll}
2 & 3
\end{array}\right][3 \quad 4]\langle 56\rangle\langle 61\rangle P_{561}^{2}\langle 5|6+1| 2]}\left(\frac{\langle 1|5+6| 3]}{\langle 1|5+6| 4]}\right)^{a}
\end{aligned}
$$




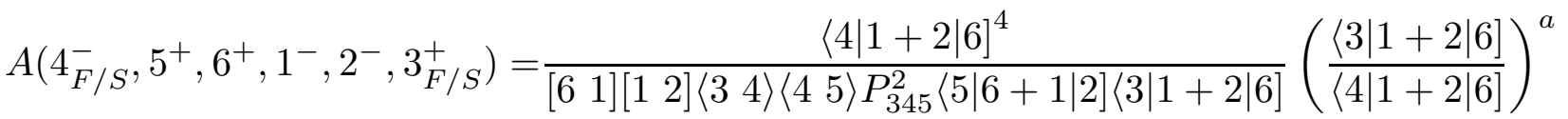

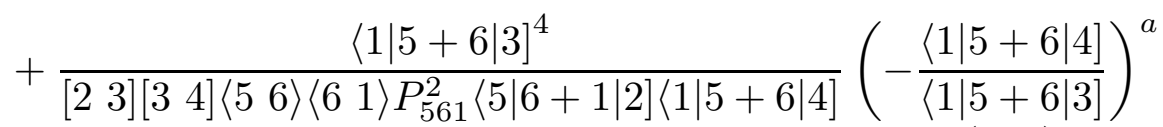

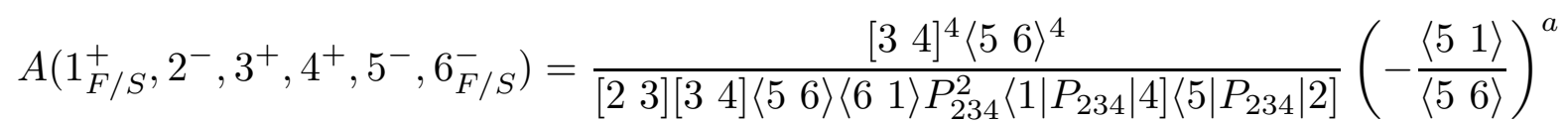

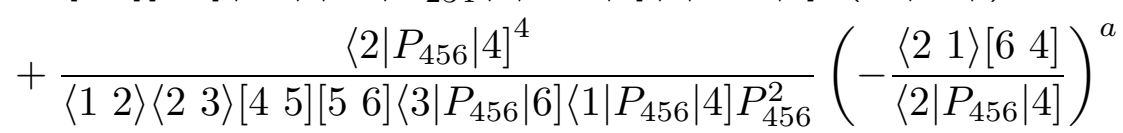

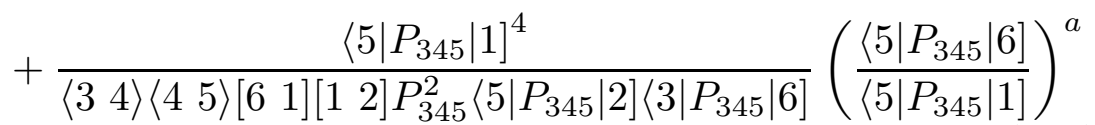

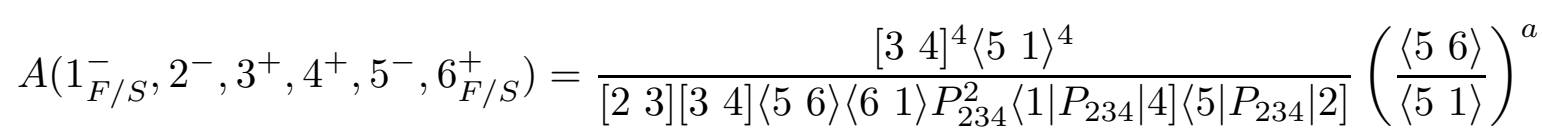

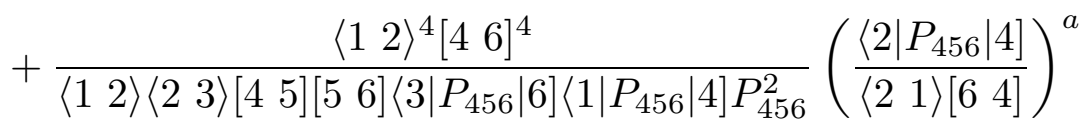

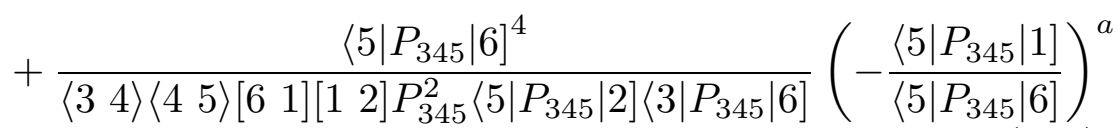

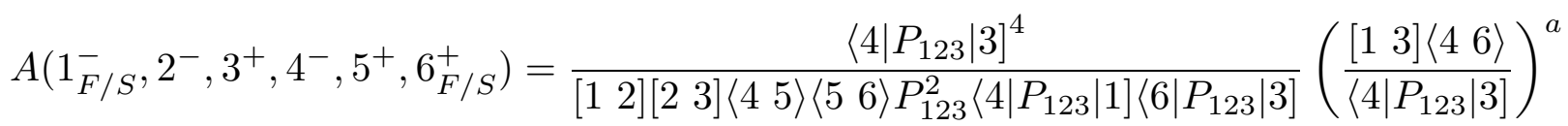

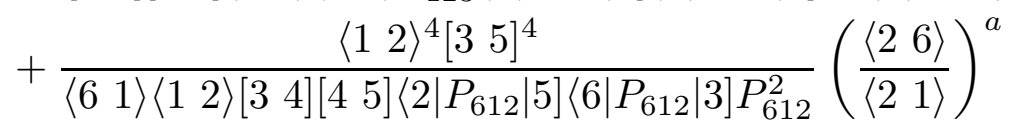

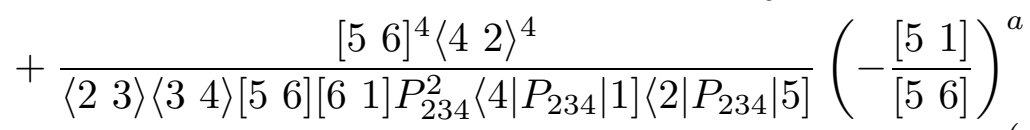

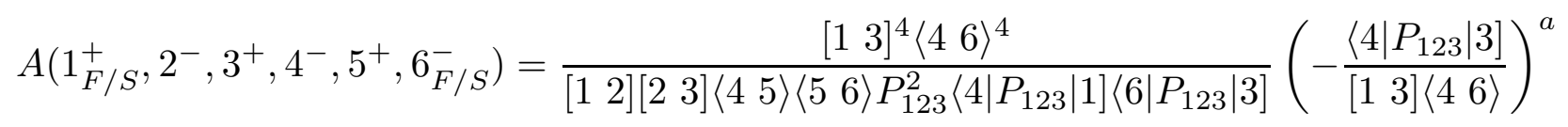

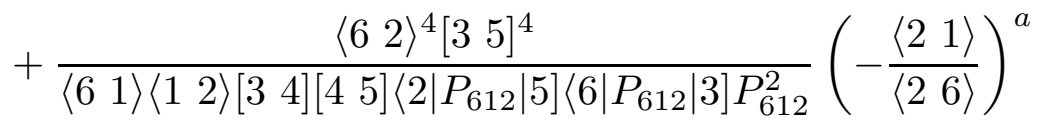

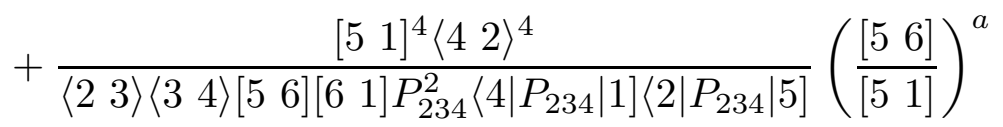


Comparing each pair $((\mathrm{A} .1),(\mathrm{A} .2)),((\mathrm{A} .3),(\mathrm{A} .4)),((\mathrm{A} .5),(\mathrm{A} .6))$, we see the pattern relating the amplitudes with opposite helicities for the fermions or scalars. In fact, this is also the reason why a factor of two appears in each cut in our scalar loops.

The expression (A.3) is different from the one given in [12]. The reason is that we have used $(2,3)$ as reference momenta in the recursion relations to derive the formula in 12 but used $(3,4)$ as reference momenta here to match the ones used in (A.4).

\section{Appendix B. Feynman Parametrization and its Integration}

Here we demonstrate in detail how to integrate terms of types (3) and (4) in the categorization (2.11). These are the integrands that give precisely the logarithmic contributions and require a Feynman parameter.

We define certain functions that we find useful. Where these appear in the paper with a tilde, this means to take the complex conjugate. We also use $\eta_{1,2}$ to denote the two solutions of the equation $\langle\ell|P Q| \ell\rangle=0$ with the proper $P, Q$ momenta, both in this and the following appendix.

\section{B.1. Type (3)}

Let us start from the following integral:

$$
T_{3}=\int\langle\ell d \ell\rangle[\ell d \ell] \frac{F(\lambda)\langle a \ell\rangle}{\left\langle\ell\left|P_{\text {cut }}\right| a\right]^{n-1}} \frac{1}{\left\langle\ell\left|P_{\text {cut }}\right| \ell\right]\left\langle\ell\left|P_{a}\right| \ell\right]} .
$$

Here we have multiplied numerator and denominator by a factor of $\langle a \ell\rangle$. To do the integration, first we introduce a Feynman parameter to rewrite $T_{3}$ as

$$
T_{3}=\int_{0}^{1} d z \int\langle\ell d \ell\rangle[\ell d \ell] \frac{F(\lambda)\langle a \ell\rangle}{\left\langle\ell\left|P_{c u t}\right| a\right]^{n-1}} \frac{1}{\langle\ell|P| \ell]^{2}}, \quad P=z P_{c u t}+(1-z) P_{a} .
$$

Next we use (2.14) to write the integrand as a derivative:

$$
T_{3}=\int_{0}^{1} d z \int\langle\ell d \ell\rangle\left[d \ell \partial_{\ell}\right]\left(\frac{F(\lambda)\langle a \ell\rangle[\widetilde{\eta} \ell]}{\left\langle\ell\left|P_{c u t}\right| a\right]^{n-1}} \frac{1}{\langle\ell|P| \ell]\langle\ell|P| \widetilde{\eta}]}\right),
$$

where $\widetilde{\eta}$ is an arbitrary but fixed spinor of negative chirality. A convenient choice is $\mid \widetilde{\eta}]=|P| a\rangle$.11 With this choice we find that

$$
T_{3}=\int_{0}^{1} d z \int\langle\ell d \ell\rangle\left[d \ell \partial_{\ell}\right]\left(-\frac{F(\lambda)\left\langle a\left|P_{c u t}\right| \ell\right]}{\left\langle\ell\left|P_{\text {cut }}\right| a\right]^{n-1}} \frac{1}{\langle\ell|P| \ell]\left(z P_{c u t}^{2}-(1-z)\left\langle a\left|P_{c u t}\right| a\right]\right)}\right) .
$$

11 There is one subtlety in the choice of $\widetilde{\eta}$. In $(\overline{B .3})$ we must avoid choosing $\mid \eta] \sim \mid a]$. The reason is that the starting point (B.1) already has a pole from the factor $[\ell a]$. In fact, if we choose $\mid \eta] \sim \mid a]$, then the integral $\int_{0}^{1} d z$ will diverge. 
Now we read out the residues of the poles. There are two kinds of poles: single poles from $F(\lambda)$ (as explained in Section 2) and the multiple pole from $\left\langle\ell\left|P_{c u t}\right| a\right]$. For residues from a single pole, the $z$-integration takes the form

$$
Z_{1}=\int_{0}^{1} d z \frac{1}{\left(a_{1}+b_{1} z\right)\left(c_{1}+d_{1} z\right)}=\frac{1}{a_{1} d_{1}-b_{1} c_{1}} \log \left(\frac{a_{1}\left(c_{1}+d_{1}\right)}{c_{1}\left(a_{1}+b_{1}\right)}\right)
$$

where $a_{1}, b_{1}, c_{1}, d_{1}$ are rational functions (for example, $a_{1}=\left\langle\ell\left|P_{a}\right| \ell\right]$ ) 12 This is always a logarithmic function. Furthermore, since it is rational, it does not have any square root which is the signature of three-mass triangle and four-mass box functions. Thus it contributes only to one-mass, two-mass and three-mass box functions.

Now we discuss the residue from the multiple pole $\left\langle\ell\left|P_{c u t}\right| a\right]^{n-1}$. One important feature of the form $(\mathbb{B . 4})$ is the factor $\left\langle a\left|P_{\text {cut }}\right| \ell\right]: 13$ it is zero precisely at the location of the pole from $\left\langle\ell\left|P_{c u t}\right| a\right]$. In other words, there is no residue contribution from the multiple pole $\left\langle\ell\left|P_{\text {cut }}\right| a\right]$ at all.14

\section{B.2. Type (4)}

The integral of type (4) is given by

$$
\begin{aligned}
T_{4} & =\int\langle\ell d \ell\rangle[\ell d \ell] \frac{F(\lambda)}{\left\langle\ell\left|P_{c u t} Q\right| \ell\right\rangle^{n-1}} \frac{1}{\left\langle\ell\left|P_{c u t}\right| \ell\right]\langle\ell|Q| \ell]} \\
& =\int_{0}^{1} d z \int\langle\ell d \ell\rangle[\ell d \ell] \frac{F(\lambda)}{\left\langle\ell\left|P_{c u t} Q\right| \ell\right\rangle^{n-1}} \frac{1}{\langle\ell|R| \ell]^{2}}, \quad R=z Q+(1-z) P_{c u t} .
\end{aligned}
$$

Similarly as for type (3), we arrive at

$$
T_{4}=\int_{0}^{1} d z \int\langle\ell d \ell\rangle\left[d \ell \partial_{\ell}\right]\left(\frac{F(\lambda)}{\left\langle\ell\left|P_{c u t} Q\right| \ell\right\rangle^{n-1}} \frac{\langle\eta|R| \ell]}{\langle\ell \eta\rangle R^{2}\langle\ell|R| \ell]}\right)
$$

12 There is a subtle point in this expression: we have assumed that there is no pole $\langle\ell a\rangle$. If this pole exists, it is easy to see that $a_{1}=0$ at pole $|\ell\rangle=|a\rangle$ and the $z$-integration diverges. We have no general argument why this is true, except to notice that there is a factor $\frac{1}{[\ell a]}$ from the anti-holomorphic part. It may be that for tree-level amplitudes, the two factors $\langle\ell a\rangle$ and $[\ell a]$ have the property that if one exists as a pole, then the other cannot.

13 Notice that since $F(\lambda)$ is a function of $\lambda$ only, the factor $\left\langle a\left|P_{c u t}\right| \ell\right]$ cannot be canceled.

14 If for some reason we have other multiple poles, we must be careful because in this case, the $z$-integration may give a rational contribution. Fortunately, in our decomposition no other multiple poles show up. 
where we have chosen the auxiliary spinor $\mid \widetilde{\eta}]=|R| \eta\rangle$.

Again we have two kinds of poles: the single poles from $F(\lambda)$ and multiple poles from the factor $\left\langle\ell\left|P_{c u t} Q\right| \ell\right\rangle^{n-1}$. Let us discuss them one by one.

\section{The Contribution from Single Poles}

The $z$-dependence comes from the momentum vector $R$, defined in (B.6) and appearing in the last factor of (B.7). It may be expressed as

$$
Z_{2}=\int_{0}^{1} d z \frac{\left(z c_{1}+c_{2}\right)}{\left(a_{0} z^{2}+a_{1} z+a_{2}\right)\left(z b_{1}+b_{2}\right)}
$$

where we have defined

$$
\begin{aligned}
& a_{0}=\left(Q-P_{\text {cut }}\right)^{2}, \quad a_{1}=2 P_{\text {cut }} \cdot\left(Q-P_{\text {cut }}\right), \quad a_{2}=P_{c u t}^{2}, \\
& b_{1}=\left\langle\ell\left|\left(Q-P_{\text {cut }}\right)\right| \ell\right], \quad b_{2}=\left\langle\ell\left|P_{\text {cut }}\right| \ell\right], \\
& c_{1}=\left\langle\eta\left|\left(Q-P_{\text {cut }}\right)\right| \ell\right], \quad c_{2}=\left\langle\eta\left|P_{\text {cut }}\right| \ell\right] .
\end{aligned}
$$

To understand the various contributions, we split the integral as follows:

$$
\begin{aligned}
Z_{2} & =\int_{0}^{1} d z \frac{\left(z c_{1}+c_{2}\right)}{\left(a_{0} z^{2}+a_{1} z+a_{2}\right)\left(z b_{1}+b_{2}\right)} \\
& =\int_{0}^{1} d z\left\{\frac{b_{1}\left(-b_{2} c_{1}+b_{1} c_{2}\right)}{\left(a_{2} b_{1}^{2}-a_{1} b_{1} b_{2}+a_{0} b_{2}^{2}\right)} \frac{1}{\left(z b_{1}+b_{2}\right)}\right. \\
& +\frac{\left(b_{2} c_{1}-b_{1} c_{2}\right)}{2\left(a_{2} b_{1}^{2}-a_{1} b_{1} b_{2}+a_{0} b_{2}^{2}\right)} \frac{\left(2 z a_{0}+a_{1}\right)}{\left(a_{0} z^{2}+a_{1} z+a_{2}\right)} \\
& \left.+\frac{\left(2 a_{2} b_{1} c_{1}-a_{1} b_{2} c_{1}-a_{1} b_{1} c_{2}+2 a_{0} b_{2} c_{2}\right)}{2\left(a_{2} b_{1}^{2}-a_{1} b_{1} b_{2}+a_{0} b_{2}^{2}\right)} \frac{1}{\left(a_{0} z^{2}+a_{1} z+a_{2}\right)}\right\}
\end{aligned}
$$

Among these three terms, the first two will give logarithmic functions with rational parameters, so these contribute to one-mass, two-mass and three-mass box functions. The third term will be

$$
\int_{0}^{1} d z \frac{1}{\left(a_{0} z^{2}+a_{1} z+a_{2}\right)}=\left.\frac{1}{\sqrt{\Delta}} \log \left(\frac{2 a_{0} z+a_{1}-\sqrt{\Delta}}{2 a_{0} z+a_{1}+\sqrt{\Delta}}\right)\right|_{0} ^{1}, \quad \Delta=a_{1}^{2}-4 a_{0} a_{2}
$$

where $\Delta$ is not a complete square, so this is not a rational function. In fact, the $\Delta$ is the characteristic signature (Gram determinant) which identifies the contribution with a particular three-mass triangle or four-mass box function. Specifically, expression (B.11) is the same as (2.6). 
The above splitting of $Z_{2}$ makes sense if and only if $a_{2} b_{1}^{2}-a_{1} b_{1} b_{2}+a_{0} b_{2}^{2} \neq 0$. From (B.9) we find that

$$
\begin{aligned}
a_{2} b_{1}^{2}-a_{1} b_{1} b_{2}+a_{0} b_{2}^{2} & =-\left\langle\ell\left|P_{\text {cut }} Q\right| \ell\right\rangle\left[\ell\left|P_{\text {cut }} Q\right| \ell\right] \\
2 a_{2} b_{1} c_{1}-a_{1} b_{2} c_{1}-a_{1} b_{1} c_{2}+2 a_{0} b_{2} c_{2} & =-\left\langle\ell\left|P_{\text {cut }} Q-Q P_{\text {cut }}\right| \eta\right\rangle\left[\ell\left|P_{\text {cut }} Q\right| \ell\right]
\end{aligned}
$$

We see that $\left(a_{2} b_{1}^{2}-a_{1} b_{1} b_{2}+a_{0} b_{2}^{2}\right)$ is zero exactly for the pole $\langle\ell|P Q| \ell\rangle$, so our manipulation is safe for single poles other than this one.

For future use, we define the following function:

$$
R_{1}\left[\ell, \eta, P_{c u t}, Q\right]=\frac{\left(2 a_{2} b_{1} c_{1}-a_{1} b_{2} c_{1}-a_{1} b_{1} c_{2}+2 a_{0} b_{2} c_{2}\right)}{2\left(a_{2} b_{1}^{2}-a_{1} b_{1} b_{2}+a_{0} b_{2}^{2}\right)}=\frac{\left\langle\ell\left|P_{c u t} Q-Q P_{c u t}\right| \eta\right\rangle}{2\left\langle\ell\left|P_{c u t} Q\right| \ell\right\rangle}
$$

The Contribution from Poles in $\langle\ell|P Q| \ell\rangle$

For the poles in $\left\langle\ell\left|P_{c u t} Q\right| \ell\right\rangle^{n-1}$, things become much more complicated. There are two facts we need to take into account. The first is that, as seen in (B.12),

$$
a_{2} b_{1}^{2}-a_{1} b_{1} b_{2}+a_{0} b_{2}^{2}=0
$$

so we need to be careful when we do the splitting. The second is that the residue will have terms like

$$
\frac{\left\langle\eta|R| \eta_{1,2}\right]\left\langle\xi|R| \eta_{1,2}\right]^{m-1}}{R^{2}\left\langle\eta_{1,2}|R| \eta_{1,2}\right]^{m}}
$$

with $m$ ranging from 1 to $(n-1)$, where $\eta_{1,2}$ are the two solutions for the poles in $\left\langle\ell\left|P_{c u t} Q\right| \ell\right\rangle^{n-1}$. In other words, we have more patterns for the $z$-integration.

Now we discuss the residues in detail for the cases $n=2,3,4$, which are the only ones needed in this paper.

\section{The case of $n=2$ :}

In this case, it is a single pole. Using (B.14) we can solve for $a_{0}$ :

$$
a_{0}=\frac{a_{1} b_{1} b_{2}-a_{2} b_{1}^{2}}{b_{2}^{2}}
$$


Therefore we can split the integral as follows:

$$
\begin{aligned}
& \int_{0}^{1} d z \frac{\left(z c_{1}+c_{2}\right)}{\left(a_{0} z^{2}+a_{1} z+a_{2}\right)\left(z b_{1}+b_{2}\right)}=\int_{0}^{1} d z \frac{b_{2}^{2}\left(z c_{1}+c_{2}\right)}{\left(a_{1} b_{2} z+a_{2} b_{2}-a_{2} b_{1} z\right)\left(z b_{1}+b_{2}\right)^{2}} \\
& =\int_{0}^{1} d z\left\{\frac{\left(a_{2} b_{2} c_{1}+a_{2} b_{1} c_{2}-a_{1} b_{2} c_{2}\right)}{b_{2}\left(2 a_{2} b_{1}-a_{1} b_{2}\right)} \frac{b_{2}^{2}}{\left(b_{1} z+b_{2}\right)\left(a_{1} b_{2} z+a_{2} b_{2}-a_{2} b_{1} z\right)}\right. \\
& \left.\quad-\frac{b_{2}\left(b_{2} c_{1}-b_{1} c_{2}\right)}{\left(2 a_{2} b_{1}-a_{1} b_{2}\right)} \frac{1}{\left(b_{2}+z b_{1}\right)^{2}}\right\} \\
& =-\int_{0}^{1} d z \frac{b_{2}\left(b_{2} c_{1}-b_{1} c_{2}\right)}{\left(2 a_{2} b_{1}-a_{1} b_{2}\right)} \frac{1}{\left(b_{2}+z b_{1}\right)^{2}}+\int_{0}^{1} d z \frac{\left(a_{2} b_{2} c_{1}+a_{2} b_{1} c_{2}-a_{1} b_{2} c_{2}\right)}{b_{2}\left(2 a_{2} b_{1}-a_{1} b_{2}\right)} \frac{1}{a_{0} z^{2}+a_{1} z+a_{2}} .
\end{aligned}
$$

Of these two terms, the first one gives a rational function while the second one gives a logarithmic function. However, we can see that

$$
b_{2} c_{1}-c_{2} b_{1}=-\langle\ell \eta\rangle\left[\ell\left|P_{c u t} Q\right| \ell\right]
$$

so that, at our pole,

$$
b_{2} c_{1}-c_{2} b_{1}=0
$$

and hence the first term vanishes. We can solve $(\mathbb{B} .16)$ for $c_{1}$ and simplify the coefficient:

$$
R_{2}\left[\ell, \eta, P_{c u t}\right]=\frac{\left(a_{2} b_{2} c_{1}+a_{2} b_{1} c_{2}-a_{1} b_{2} c_{2}\right)}{b_{2}\left(2 a_{2} b_{1}-a_{1} b_{2}\right)}=\frac{c_{2}}{b_{2}}=\frac{\left\langle\eta\left|P_{c u t}\right| \ell\right]}{\left\langle\ell\left|P_{c u t}\right| \ell\right]}
$$

Unlike the function $R_{1}$ defined in (B.13) in which $Q$ appears explicitly, $R_{2}$ does not involve $Q$. However, in the formula for the coefficient, $R_{2}$ will depend on $Q$ through $\ell$ when evaluated at $\eta_{1,2}$, the solutions of $\left\langle\ell\left|P_{c u t} Q\right| \ell\right\rangle=0$.

\section{The case of $n=3$ :}

For the case $n=3$ we have a double pole. Using our residue formula (2.19) we find that the new $z$-integral may be expressed as

$$
Z_{3}=\int_{0}^{1} d z \frac{\left(z c_{1}+c_{2}\right)\left(z d_{1}+d_{2}\right)}{\left(a_{0} z^{2}+a_{1} z+a_{2}\right)\left(z b_{1}+b_{2}\right)^{2}}
$$

where $a_{i}, b_{i}, c_{i}$ are the same as in $(\mathrm{B.9})$, and

$$
d_{1}=\left\langle\xi\left|\left(Q-P_{\text {cut }}\right)\right| \ell\right], \quad d_{2}=\left\langle\xi\left|P_{\text {cut }}\right| \ell\right] .
$$


By calculations similar to those in the case of $n=2$, we reach

$$
\begin{aligned}
Z_{3} & =\int_{0}^{1} d z \frac{b_{2}^{2}\left(z c_{1}+c_{2}\right)\left(z d_{1}+d_{2}\right)}{\left(a_{1} b_{2} z-a_{2} b_{1} z+a_{2} b_{2}\right)\left(z b_{1}+b_{2}\right)^{3}} \\
& =\frac{c_{1}\left(-b_{2} d_{1}+b_{1} d_{2}\right)}{b_{1}\left(b_{1}+b_{2}\right)\left(2 a_{2} b_{1}-a_{1} b_{2}\right)} \\
& +\frac{c_{1}\left(a_{2} b_{2} d_{1}+a_{2} b_{1} d_{2}-a_{1} b_{2} d_{2}\right)}{b_{2} b_{1}\left(2 a_{2} b_{1}-a_{1} b_{2}\right)} \int_{0}^{1} \frac{b_{2}^{2}}{\left(b_{1} z+b_{2}\right)\left(a_{1} b_{2} z+a_{2} b_{2}-a_{2} b_{1} z\right)}
\end{aligned}
$$

where we have used the condition $(\overline{B .16})$ to simplify the result. We see that

$$
-b_{2} d_{1}+b_{1} d_{2}=\langle\ell \xi\rangle[\ell|P Q| \ell]
$$

so that at our pole

$$
-b_{2} d_{1}+b_{1} d_{2}=0
$$

so the rational contribution is zero. The relevant nonzero coefficient is given by

$$
\begin{aligned}
R_{3}\left[\ell, \eta, \xi, P_{c u t}\right] & =\frac{c_{1}\left(a_{2} b_{2} d_{1}+a_{2} b_{1} d_{2}-a_{1} b_{2} d_{2}\right)}{b_{2} b_{1}\left(2 a_{2} b_{1}-a_{1} b_{2}\right)} \\
& =-\frac{\left\langle\eta\left|\left(Q-P_{c u t}\right)\right| \ell\right]\left\langle\xi\left|P_{c u t}\left(P_{c u t} Q+Q P_{c u t}\right)\right| \ell\right]}{\left\langle\ell\left|\left(Q-P_{c u t}\right)\right| \ell\right]\left(2 P_{c u t}^{2}\langle\ell|Q| \ell]-2 P_{c u t} \cdot Q\left\langle\ell\left|P_{c u t}\right| \ell\right]\right)}
\end{aligned}
$$

Using the results $(\mathbb{B . 1 6})$ and $(\mathbb{B} .20)$, this can be simplified to the formula

$$
R_{3}\left[\ell, \eta, \xi, P_{c u t}\right]=\frac{c_{2} d_{2}}{b_{2}^{2}}=\frac{\left\langle\eta\left|P_{c u t}\right| \ell\right]\left\langle\xi\left|P_{c u t}\right| \ell\right]}{\left\langle\ell\left|P_{c u t}\right| \ell\right]^{2}}
$$

The case of $n=4$ :

For $n=4$ we have a triple pole; the new $z$-integration pattern is given by

$$
Z_{4}=\int_{0}^{1} d z \frac{\left(z c_{1}+c_{2}\right)\left(z d_{1}+d_{2}\right)^{2}}{\left(a_{0} z^{2}+a_{1} z+a_{2}\right)\left(z b_{1}+b_{2}\right)^{3}}
$$

where $a_{i}, b_{i}, c_{i}, d_{i}$ are given above in $(\mathrm{B} .9)$ and (B.18).

Now we perform similar manipulations as in the case $n=3$. Using the three zeroconditions (B.14), (B.16), and (B.20), we find once again that the rational contribution is zero and we are left with

$$
Z_{4}=R_{4} \int_{0}^{1} d z \frac{1}{a_{0} z^{2}+a_{1} z+a_{2}}
$$


where

$$
R_{4}\left[\ell, \eta, \xi, P_{c u t}\right]=\frac{c_{2} d_{2}^{2}}{b_{2}^{3}}=\frac{\left\langle\eta\left|P_{c u t}\right| \ell\right]\left\langle\xi\left|P_{c u t}\right| \ell\right]^{2}}{\left\langle\ell\left|P_{\text {cut }}\right| \ell\right]^{3}} .
$$

For general situations, it can be done by similar way. Observing above patterns for $R_{2}, R_{3}, R_{4}$ we conjecture that for the pattern

$$
Z_{n+1} \equiv \int_{0}^{1} d z \frac{\prod_{i=1}^{n}\left\langle\xi_{i}|R| \ell\right]}{R^{2}\langle\ell|R| \ell]^{n}}
$$

the contribution of poles of the solution $\left\langle\ell\left|P_{c u t} Q\right| \ell\right\rangle=0$ to three-mass triangles or fourmass boxes will be given by

$$
R_{n+1}\left[\ell,\left\{\xi_{1}, \ldots, \xi_{n}\right\}, P_{c u t}\right] \equiv \frac{\prod_{i=1}^{n}\left\langle\xi_{i}\left|P_{c u t}\right| \ell\right]}{\left\langle\ell\left|P_{c u t}\right| \ell\right]^{n}}
$$

\section{Appendix C. Coefficients of Three-Mass Triangles}

In this appendix we discuss the presentation of coefficients of three-mass triangles. After the $t$-integration and splitting we end up with an integral of the form

$$
S_{3}=\int\langle\ell d \ell\rangle[\ell d \ell] \frac{1}{\langle\ell|P| \ell]\langle\ell|Q| \ell]} \frac{\prod_{i=1}^{N}\left\langle\ell a_{i}\right\rangle}{\langle\ell|P Q| \ell\rangle^{(N-m) / 2} \prod_{j=1}^{m}\left\langle\ell b_{j}\right\rangle},
$$

where the factor $\langle\ell|P Q| \ell\rangle$ is special to this case (here $P$ is the $P_{c u t}$ in Appendix $\mathrm{B}$, but for simplicity we write $P$ in this part).

The first thing is to write it using Feynman parametrization as

$$
\begin{aligned}
& S_{3}=\int_{0}^{1} d z \int\langle\ell d \ell\rangle[\ell d \ell] \frac{1}{\langle\ell|R| \ell]^{2}} \frac{\prod_{i=1}^{N}\left\langle\ell a_{i}\right\rangle}{\langle\ell|P Q| \ell\rangle^{(N-m) / 2} \prod_{j=1}^{m}\left\langle\ell b_{j}\right\rangle}, \quad R=z Q+(1-z) P, \\
& =\int_{0}^{1} d z \int\langle\ell d \ell\rangle\left[d \ell \partial_{\ell}\right] \frac{[\widetilde{\eta} \ell]}{\langle\ell|R| \ell]\langle\ell|R| \widetilde{\eta}]} \frac{\prod_{i=1}^{N}\left\langle\ell a_{i}\right\rangle}{\langle\ell|P Q| \ell\rangle^{(N-m) / 2} \prod_{j=1}^{m}\left\langle\ell b_{j}\right\rangle},
\end{aligned}
$$

where $\widetilde{\eta}$ is an arbitrary auxiliary spinor of negative chirality. In this form we have a pole at $|R| \widetilde{\eta}\rangle$, which depends on $z$. To simplify calculations, we take $\mid \widetilde{\eta}]=|R| \eta\rangle$ so that

$$
S_{3}=\int_{0}^{1} d z \int\langle\ell d \ell\rangle\left[d \ell \partial_{\ell}\right] \frac{\langle\eta|R| \ell]}{\langle\ell|R| \ell] R^{2}\langle\ell \eta\rangle} \frac{\prod_{i=1}^{N}\left\langle\ell a_{i}\right\rangle}{\langle\ell|P Q| \ell\rangle^{(N-m) / 2} \prod_{j=1}^{m}\left\langle\ell b_{j}\right\rangle} .
$$

The reason for this choice is that now all poles are independent of $z$ (notice the extra pole $\langle\ell \eta\rangle)$; all the $z$-dependence is in the first factor. 
First, we deal with the single poles at $b_{i}, \eta$. For a single pole, the $z$-integration pattern is given by $Z_{2}$ and $R_{1}$ defined in Appendix B. Thus we get the following result:

$$
C_{3}^{(\text {sing })}=-\sum_{p=1}^{m+1} \frac{\prod_{i=1}^{N}\left\langle b_{p} a_{i}\right\rangle}{\left\langle b_{p}|P Q| b_{p}\right\rangle^{(N-m) / 2} \prod_{j=1}^{\prime m+1}\left\langle b_{p} b_{j}\right\rangle} R_{1}\left[b_{p}, \eta, P, Q\right]
$$

There are several remarks about these results: (1) the minus sign appears because we need to take the negative residues of poles; (2) we have set $b_{m+1}=\eta ;(3)$ in the denominator, the prime symbol / means that we need to omit the factor with $j=p$ in the product; (4) the factor $R_{1}\left[b_{p}, \eta, P, Q\right]$ is the contribution of the $z$-integration defined by $(\overline{\mathrm{B} .13})$.

Now we need to deal with the pole from $\langle\ell|P Q| \ell\rangle^{(N-m) / 2}$. We proceed case by case. If $(N-m) / 2=0$ there is no such pole. The next case is that $(N-m) / 2=1$, i.e., two single poles. Then we have the following result:

$$
S_{3 ; 1}=-\sum_{p=1,2} \lim _{\ell \rightarrow \eta_{p}} \frac{\left\langle\ell \eta_{p}\right\rangle}{\langle\ell|P Q| \ell\rangle} \frac{\prod_{i=1}^{N}\left\langle\ell a_{i}\right\rangle}{\langle\ell \eta\rangle \prod_{j=1}^{N-2}\left\langle\ell b_{j}\right\rangle} R_{2}[\ell, \eta, P]
$$

where $\eta_{1,2}$ are solutions of $\langle\ell|P Q| \ell\rangle=0$ and $R_{2}$ is defined by (B.17). We will stick to the notation $\eta_{1,2}$.

Now we proceed to the case $(N-m) / 2=2$, i.e., double poles. Using

$$
\langle\ell|P Q| \ell\rangle=\left\langle\ell \eta_{1}\right\rangle\left\langle\ell \eta_{2}\right\rangle \frac{\langle b|P Q| b\rangle}{\langle a b\rangle^{2}}
$$

where $a, b$ are two spinors used to solve $\eta_{1}, \eta_{2}$ (see also equations (4.19)), we get the following expression:

$$
S_{3}=\int_{0}^{1} d z \int\langle\ell d \ell\rangle\left[d \ell \partial_{\ell}\right] \frac{\langle\eta|R| \ell]}{\langle\ell|R| \ell] R^{2}\langle\ell \eta\rangle} \frac{\prod_{i=1}^{N}\left\langle\ell a_{i}\right\rangle}{\left\langle\ell \eta_{1}\right\rangle^{2}\left\langle\ell \eta_{2}\right\rangle^{2} \frac{\langle b|P Q| b\rangle^{2}}{\langle a b\rangle^{4}} \prod_{j=1}^{N-4}\left\langle\ell b_{j}\right\rangle}
$$

Now using the formula (2.19) for the residue of double poles, we find (here the limit is more like replacement, but we use this notation to simplify our discussion)

$$
\begin{aligned}
& -\lim _{\ell \rightarrow \eta_{1}} \frac{\langle\eta|R| \ell]}{\langle\ell|R| \ell] R^{2}\langle\ell \eta\rangle} \frac{\prod_{i=1}^{N}\left\langle\ell a_{i}\right\rangle}{\left\langle\ell \eta_{2}\right\rangle^{2} \frac{\langle b|P Q| b\rangle^{2}}{\langle a b\rangle^{4}} \prod_{j=1}^{N-4}\left\langle\ell b_{j}\right\rangle}\left(\sum_{i=1}^{N-1} \frac{\left\langle L_{1 i} L_{2 i}\right\rangle}{\left\langle\eta_{1} L_{1 i}\right\rangle\left\langle\eta_{1} L_{2 i}\right\rangle}+\frac{\left\langle a_{N}|R| \eta_{1}\right]}{\left\langle\eta_{1}|R| \eta_{1}\right]\left\langle\eta_{1} a_{N}\right\rangle}\right) \\
& -\left\{\eta_{1} \rightarrow \eta_{2}, L_{2} \rightarrow L_{3}\right\}
\end{aligned}
$$


with

$$
\begin{aligned}
& L_{1}=\left\{a_{1}, a_{2}, \ldots, a_{N}\right\} \\
& \left.L_{2}=\left\{b_{1}, b_{2}, \ldots, b_{N-4}, \eta, \eta_{2}, \eta_{2},|R| \eta_{1}\right]\right\} \\
& \left.L_{3}=\left\{b_{1}, b_{2}, \ldots, b_{N-4}, \eta, \eta_{1}, \eta_{1},|R| \eta_{2}\right]\right\} .
\end{aligned}
$$

There are two terms. The first term is the contribution of double pole $\eta_{1}$ and the second term is that of $\eta_{2}$. We got the latter from the former by replacing $\eta_{1} \rightarrow \eta_{2}$ and $L_{2} \rightarrow L_{3}$. In the first line, the most significant manipulation is that in the brackets we have separated $N$ terms into two parts: the first $N-1$ terms are independent of $R$, while the last term depends on $R$ and thus on $z$. This will make the $z$-integration different as we will see shortly.

Having residues given by (C.6) we need to do the Feynman parameter integration and read out contribution to three-mass triangles. This has essentially been done in Appendix B. Using (B.17) and (B.22), we get

$$
\begin{aligned}
& -\lim _{\ell \rightarrow \eta_{1}} \frac{\prod_{i=1}^{N}\left\langle\ell a_{i}\right\rangle}{\langle\ell \eta\rangle\left\langle\ell \eta_{2}\right\rangle^{2} \frac{\langle b|P Q| b\rangle^{2}}{\langle a b\rangle^{4}} \prod_{j=1}^{N-4}\left\langle\ell b_{j}\right\rangle}\left(\sum_{i=1}^{N-1} \frac{\left\langle L_{1 i} L_{2 i}\right\rangle}{\left\langle\ell L_{1 i}\right\rangle\left\langle\ell L_{2 i}\right\rangle} R_{2}[\ell, \eta, P]\right. \\
& \left.+\frac{1}{\left\langle\ell a_{N}\right\rangle} R_{3}\left[\ell, \eta, a_{N}, P\right]\right)-\left\{\eta_{1} \rightarrow \eta_{2}, L_{2} \rightarrow L_{3}\right\}
\end{aligned}
$$

Now we discuss the last case needed for our calculation, namely $(N-m) / 2=3$. We have

$$
S_{3}=\int_{0}^{1} d z \int\langle\ell d \ell\rangle\left[d \ell \partial_{\ell}\right] \frac{\langle\eta|R| \ell]}{\langle\ell|R| \ell] R^{2}\langle\ell \eta\rangle} \frac{\prod_{i=1}^{N}\left\langle\ell a_{i}\right\rangle}{\left\langle\ell \eta_{1}\right\rangle^{3}\left\langle\ell \eta_{2}\right\rangle^{3} \frac{\langle b|P Q| b\rangle^{3}}{\langle a b\rangle^{6}} \prod_{j=1}^{N-6}\left\langle\ell b_{j}\right\rangle} .
$$

Here we have two triple poles. Again we need to read out the negative residue first and then use previous results to get the coefficients. For a triple pole $\eta_{1}$ there are several parts in the denominator: (1) factors with $b_{i}, i=1, \ldots, N-6,(2)$ one factor with $\eta$, (3) three factors with $\eta_{2}$, and (4) one factor with $\left.|R| \eta_{1}\right]$. We group the first three together into the list $L_{b, 1}^{I V}$ defined below. The reason doing that is that only the last one has $z$-dependence. 
Using this notation we find that the residue of $\eta_{1}$ is given by

$$
\begin{aligned}
& -\frac{\left\langle\eta_{1}|R| \eta_{1}\right]}{\left\langle\eta_{1}|R| \eta_{1}\right] R^{2}\left\langle\eta_{1} L_{b, 1, N-5}^{I V}\right\rangle} \frac{\prod_{i=1}^{N}\left\langle\eta_{1} L_{a, i}\right\rangle}{\left\langle\eta_{1} \eta_{2}\right\rangle^{3} \frac{\langle b|P Q| b\rangle^{3}}{\langle a b\rangle^{6}} \prod_{j=1}^{N-6}\left\langle\eta_{1} L_{b, 1, j}^{I V}\right\rangle} \\
& {\left[\sum_{i=1}^{N-2} \frac{\left\langle L_{a, i} L_{b, 1, i}^{I V}\right\rangle}{\left\langle\eta_{1} L_{a, i}\right\rangle\left\langle\eta_{1} L_{b, 1, i}^{I V}\right\rangle} \frac{\left\langle L_{a, N} L_{a, i}\right\rangle}{\left\langle\eta_{1} L_{a, N}\right\rangle\left\langle\eta_{1} L_{a, i}\right\rangle}\right.} \\
& +\frac{\left\langle a_{N-1}|R| \eta_{1}\right]}{\left\langle\eta_{1}|R| \eta_{1}\right]\left\langle\eta_{1} a_{N-1}\right\rangle} \frac{\left\langle a_{N} a_{N-1}\right\rangle}{\left\langle\eta_{1} a_{N}\right\rangle\left\langle\eta_{1} a_{N-1}\right\rangle}+\sum_{1 \leq i \leq j \leq N-2} \frac{\left\langle L_{a, i} L_{b, 1, i}^{I V}\right\rangle}{\left\langle\eta_{1} L_{a, i}\right\rangle\left\langle\eta_{1} L_{b, 1, i}^{I V}\right\rangle} \frac{\left\langle L_{a, j} L_{b, 1, j}^{I V}\right\rangle}{\left\langle\eta_{1} L_{a, j}\right\rangle\left\langle\eta_{1} L_{b, 1, j}^{I V}\right\rangle} \\
& \left.+\sum_{1 \leq i \leq N-2} \frac{\left\langle L_{a, i} L_{b, 1, i}^{I V}\right\rangle}{\left\langle\eta_{1} L_{a, i}\right\rangle\left\langle\eta_{1} L_{b, 1, i}^{I V}\right\rangle} \frac{\left\langle L_{a, N-1}|R| \eta_{1}\right]}{\left\langle\eta_{1} L_{a, N-1}\right\rangle\left\langle\eta_{1}|R| \eta_{1}\right]}+\left(\frac{\left\langle L_{a, N-1}|R| \eta_{1}\right]}{\left\langle\eta_{1} L_{a, N-1}\right\rangle\left\langle\eta_{1}|R| \eta_{1}\right]}\right)^{2}\right]
\end{aligned}
$$

In this result, we have separated terms having different $R$ factors. From this we can read the coefficient of the three-mass triangle as

$$
\begin{aligned}
& \frac{-\prod_{i=1}^{N}\left\langle\eta_{1} L_{a, i}\right\rangle}{\left\langle\eta_{1} L_{b, 1, N-5}^{I V}\right\rangle\left\langle\eta_{1} \eta_{2}\right\rangle^{3} \frac{\left\langle b|P Q| b b^{3}\right.}{\langle a b\rangle^{6}} \prod_{j=1}^{N-6}\left\langle\eta_{1} L_{b, 1, j}^{I V}\right\rangle}\left[R_{2}\left[\eta_{1}, L_{b, 1, N-5}^{I V}, P\right]\right. \\
& \left(\sum_{i=1}^{N-2} \frac{\left\langle L_{a, i} L_{b, 1, i}^{I V}\right\rangle}{\left\langle\eta_{1} L_{a, i}\right\rangle\left\langle\eta_{1} L_{b, 1, i}^{I V}\right\rangle} \frac{\left\langle L_{a, N} L_{a, i}\right\rangle}{\left\langle\eta_{1} L_{a, N}\right\rangle\left\langle\eta_{1} L_{a, i}\right\rangle}\right. \\
& \left.+\sum_{1 \leq i \leq j \leq N-2} \frac{\left\langle L_{a, i} L_{b, 1, i}^{I V}\right\rangle}{\left\langle\eta_{1} L_{a, i}\right\rangle\left\langle\eta_{1} L_{b, 1, i}^{I V}\right\rangle} \frac{\left\langle L_{a, j} L_{b, 1, j}^{I V}\right\rangle}{\left\langle\eta_{1} L_{a, j}\right\rangle\left\langle\eta_{1} L_{b, 1, j}^{I V}\right\rangle}\right) \\
& +R_{3}\left[\eta_{1}, L_{b, N-5}^{I V}, L_{a, N-1}, P\right] \\
& \left(\frac{\left\langle L_{a, N} L_{a, N-1}\right\rangle}{\left\langle\eta_{1} L_{a, N-1}\right\rangle\left\langle\eta_{1} L_{a, N}\right\rangle\left\langle\eta_{1} L_{a, N-1}\right\rangle}+\sum_{1 \leq i \leq N-2} \frac{\left\langle L_{a, i} L_{b, 1, i}^{I V}\right\rangle}{\left\langle\eta_{1} L_{a, N-1}\right\rangle\left\langle\eta_{1} L_{a, i}\right\rangle\left\langle\eta_{1} L_{b, 1, i}^{I V}\right\rangle}\right) \\
& \left.+\frac{1}{\left\langle\eta_{1} L_{a, N-1}\right\rangle^{2}} R_{4}\left[\eta_{1}, L_{b, N-5}^{I V}, L_{a, N-1}, P\right]\right] .
\end{aligned}
$$

\section{Summary:}

The reader who wishes to skip the derivations may simply use the formulas given below. Where these functions appear in the bulk of the paper with a tilde, this means to take the complex conjugate. 
We define the following lists.

$$
\begin{aligned}
L_{a} & =\left\{a_{1}, a_{2}, \ldots, a_{N}\right\}, \\
L_{b}^{I} & =\left\{b_{1}, b_{2}, \ldots, b_{N}, \eta\right\}, \\
L_{b}^{I I} & =\left\{b_{1}, b_{2}, \ldots, b_{N-2}, \eta\right\}, \\
L_{b, 1}^{I I I} & =\left\{b_{1}, b_{2}, \ldots, b_{N-4}, \eta, \eta_{2}, \eta_{2}\right\}, \\
L_{b, 2}^{I I I} & =\left\{b_{1}, b_{2}, \ldots, b_{N-4}, \eta, \eta_{1}, \eta_{1}\right\}, \\
L_{b, 1}^{I V} & =\left\{b_{1}, b_{2}, \ldots, b_{N-6}, \eta, \eta_{2}, \eta_{2}, \eta_{2}\right\}, \\
L_{b, 2}^{I V} & =\left\{b_{1}, b_{2}, \ldots, b_{N-6}, \eta, \eta_{1}, \eta_{1}, \eta_{1}\right\},
\end{aligned}
$$

where $\eta$ is an arbitrary auxiliary spinor.

(1) Case one: for the integral

$$
S_{3}^{I}=\int\langle\ell d \ell\rangle[\ell d \ell] \frac{1}{\langle\ell|P| \ell]\langle\ell|Q| \ell]} \frac{\prod_{i=1}^{N}\left\langle\ell a_{i}\right\rangle}{\prod_{j=1}^{N}\left\langle\ell b_{j}\right\rangle}
$$

we have the coefficient

$$
C_{3}^{I}\left[L_{a}, L_{b}^{I}, P, Q\right]=\sum_{p=1}^{N+1} \frac{-\prod_{i=1}^{N}\left\langle L_{b, p}^{I} L_{a, i}\right\rangle}{\prod_{j=1}^{\prime N+1}\left\langle L_{b, p}^{I} L_{b, j}^{I}\right\rangle} R_{1}\left[L_{b, p}^{I}, L_{b, N+1}^{I}, P, Q\right] .
$$

Although in this paper we have not encountered this situation, we include it for completeness.

(2) Case two: for the integral

$$
S_{3}=\int\langle\ell d \ell\rangle[\ell d \ell] \frac{1}{\langle\ell|P| \ell]\langle\ell|Q| \ell]} \frac{\prod_{i=1}^{N}\left\langle\ell a_{i}\right\rangle}{\langle\ell|P Q| \ell\rangle \prod_{j=1}^{N-2}\left\langle\ell b_{j}\right\rangle},
$$

we have the coefficient

$$
\begin{aligned}
C_{3}^{I I}\left[L_{a}, L_{b}^{I I}, P, Q\right]= & \sum_{p=1}^{N-1} \frac{-\prod_{i=1}^{N}\left\langle L_{b, p}^{I I} L_{a, i}\right\rangle}{\left\langle L_{b, p}^{I I}|P Q| L_{b, p}^{I I}\right\rangle \prod_{j=1}^{N-1}\left\langle L_{b, p}^{I I} L_{b, j}^{I I}\right\rangle} R_{1}\left[L_{b, p}^{I I}, L_{b, N-1}^{I I}, P, Q\right] \\
& -\sum_{p=1,2} \lim _{\ell \rightarrow \eta_{p}} \frac{\left\langle\ell \eta_{p}\right\rangle}{\langle\ell|P Q| \ell\rangle} \frac{\prod_{i=1}^{N}\left\langle\ell L_{a, i}\right\rangle}{\left\langle\ell L_{b, N-1}^{I I}\right\rangle \prod_{j=1}^{N-2}\left\langle\ell L_{b, j}^{I I}\right\rangle} R_{2}\left[\ell, L_{b, N-1}^{I I}, P\right] .
\end{aligned}
$$

(3) Case three: for the integral

$$
S_{3}=\int\langle\ell d \ell\rangle[\ell d \ell] \frac{1}{\langle\ell|P| \ell]\langle\ell|Q| \ell]} \frac{\prod_{i=1}^{N}\left\langle\ell a_{i}\right\rangle}{\langle\ell|P Q| \ell\rangle^{2} \prod_{j=1}^{N-4}\left\langle\ell b_{j}\right\rangle},
$$


we have the coefficient

$$
\begin{aligned}
& C_{3}^{I I I}\left[L_{a}, L_{b, 1}^{I I I}, L_{b, 2}^{I I I}, P, Q\right] \\
& =\sum_{p=1}^{N-3} \frac{-\prod_{i=1}^{N}\left\langle L_{b, p}^{I I I} L_{a, i}\right\rangle}{\left\langle L_{b, p}^{I I I}|P Q| L_{b, p}^{I I}\right\rangle^{2} \prod_{j=1}^{\prime N-3}\left\langle L_{b, p}^{I I I} L_{b, j}^{I I I}\right\rangle} R_{1}\left[L_{b, 1, p}^{I I I}, P, Q, L_{b, 1, N-3}^{I I I}\right] \\
& -\left[\lim _{\ell \rightarrow \eta_{1}} \frac{\prod_{i=1}^{N}\left\langle\ell L_{a, i}\right\rangle}{\left\langle\ell L_{b, 1, N-3}^{I I I}\right\rangle\left\langle\ell \eta_{2}\right\rangle^{2} \frac{\langle b|P Q| b\rangle^{2}}{\langle a b\rangle^{4}} \prod_{j=1}^{N-4}\left\langle\ell L_{b, 1, j}^{I I I}\right\rangle}\right. \\
& \left(R_{2}\left[\ell, L_{b, 1, N-3}^{I I I}, P\right] \sum_{i=1}^{N-1} \frac{\left\langle L_{a, i} L_{b, 1, i}^{I I I}\right\rangle}{\left\langle\eta_{1} L_{a, i}\right\rangle\left\langle\eta_{1} L_{b, 1, i}^{I I I}\right\rangle}+\frac{1}{\left\langle\eta_{1} L_{a, N}\right\rangle} R_{3}\left[\eta_{1}, L_{b, 1, N-3}^{I I I}, L_{a, N}, P\right]\right) \\
& \left.+\left\{\eta_{1} \rightarrow \eta_{2}, L_{b, 1}^{I I I} \rightarrow L_{b, 2}^{I I I}\right\}\right] .
\end{aligned}
$$

(4) Case four: for the integral

$$
S_{3}=\int\langle\ell d \ell\rangle[\ell d \ell] \frac{1}{\langle\ell|P| \ell]\langle\ell|Q| \ell]} \frac{\prod_{i=1}^{N}\left\langle\ell a_{i}\right\rangle}{\langle\ell|P Q| \ell\rangle^{3} \prod_{j=1}^{N-6}\left\langle\ell b_{j}\right\rangle},
$$

we have the coefficient

$$
\begin{aligned}
& C_{3}^{I V}\left[L_{a}, L_{b, 1}^{I V}, L_{b, 2}^{I V}, P, Q\right] \\
& =\sum_{p=1}^{N-5} \frac{-\prod_{i=1}^{N}\left\langle L_{b, p}^{I V} L_{a, i}\right\rangle}{\left\langle L_{b, p}^{I V}|P Q| L_{b, p}^{I V}\right\rangle^{3} \prod_{j=1}^{\prime N-5}\left\langle L_{b, p}^{I V} L_{b, j}^{I V}\right\rangle} R_{1}\left[L_{b, 1, p}^{I V}, P, Q, L_{b, 1, N-5}^{I V}\right] \\
& -\left[\frac { \prod _ { i = 1 } ^ { N } \langle \eta _ { 1 } L _ { a , i } \rangle } { \langle \eta _ { 1 } L _ { b , 1 , N - 5 } ^ { I V } \rangle \langle \eta _ { 1 } \eta _ { 2 } \rangle ^ { 3 } \frac { \langle b | P Q | b b ^ { 3 } } { \langle a b \rangle ^ { 6 } } \prod _ { j = 1 } ^ { N - 6 } \langle \eta _ { 1 } L _ { b , 1 , j } ^ { I V } \rangle } \left(R_{2}\left[\eta_{1}, L_{b, 1, N-5}^{I V}, P\right]\right.\right. \\
& \left(\sum_{i=1}^{N-2} \frac{\left\langle L_{a, i} L_{b, 1, i}^{I V}\right\rangle}{\left\langle\eta_{1} L_{a, i}\right\rangle\left\langle\eta_{1} L_{b, 1, i}^{I V}\right\rangle} \frac{\left\langle L_{a, N} L_{a, i}\right\rangle}{\left\langle\eta_{1} L_{a, N}\right\rangle\left\langle\eta_{1} L_{a, i}\right\rangle}\right. \\
& \left.+\sum_{1 \leq i \leq j \leq N-2} \frac{\left\langle L_{a, i} L_{b, 1, i}^{I V}\right\rangle}{\left\langle\eta_{1} L_{a, i}\right\rangle\left\langle\eta_{1} L_{b, 1, i}^{I V}\right\rangle} \frac{\left\langle L_{a, j} L_{b, 1, j}^{I V}\right\rangle}{\left\langle\eta_{1} L_{a, j}\right\rangle\left\langle\eta_{1} L_{b, 1, j}^{I V}\right\rangle}\right) \\
& +\left(\frac{\left\langle L_{a, N} L_{a, N-1}\right\rangle}{\left\langle\eta_{1} L_{a, N-1}\right\rangle\left\langle\eta_{1} L_{a, N}\right\rangle\left\langle\eta_{1} L_{a, N-1}\right\rangle}+\sum_{1 \leq i \leq N-2} \frac{\left\langle L_{a, i} L_{b, 1, i}^{I V}\right\rangle}{\left\langle\eta_{1} L_{a, N-1}\right\rangle\left\langle\eta_{1} L_{a, i}\right\rangle\left\langle\eta_{1} L_{b, 1, i}^{I V}\right\rangle}\right) \\
& \left.R_{3}\left[\eta_{1}, L_{b, N-5}^{I V}, L_{a, N-1}, P\right]+\frac{1}{\left\langle\eta_{1} L_{a, N-1}\right\rangle^{2}} R_{4}\left[\eta_{1}, L_{b, N-5}^{I V}, L_{a, N-1}, P\right]\right) \\
& \left.+\left\{\eta_{1} \rightarrow \eta_{2}, L_{b, 1}^{I V} \rightarrow L_{b, 2}^{I V}\right\}\right] .
\end{aligned}
$$




\section{References}

[1] G. P. Salam, "Developments in perturbative QCD," arXiv:hep-ph/0510090.

[2] F. Gianotti and M. L. Mangano, "LHC physics: The first one-two year(s)," arXiv:hepph/0504221.

[3] Z. Bern, L. J. Dixon and D. A. Kosower, "One Loop Corrections To Five Gluon Amplitudes," Phys. Rev. Lett. 70, 2677 (1993), hep-ph/9302280. Z. Kunszt, A. Signer and Z. Trocsanyi, "One loop radiative corrections to the helicity amplitudes of QCD processes involving four quarks and one gluon," Phys. Lett. B 336 (1994) 529 hepph/9405386]; Z. Bern, L.J. Dixon and D.A. Kosower, "One loop corrections to two quark three gluon amplitudes," Nucl. Phys. B 437 (1995) 259 hep-ph/9409393;

[4] A. Denner, S. Dittmaier, M. Roth and L. H. Wieders, "Complete electroweak O(alpha) corrections to charged-current e+ e- $\rightarrow$ Phys. Lett. B 612, 223 (2005) arXiv:hepph/0502063 . A. Denner, S. Dittmaier, M. Roth and L. H. Wieders, "Electroweak corrections to charged-current e+ e- $\rightarrow 4$ fermion processes: Technical details and further results," arXiv:hep-ph/0505042. F. Boudjema, et al.", "Electroweak corrections for the study of the Higgs potential at the LC", arXiv:hep-ph/0510184

[5] A. Denner, and S. Dittmaier, "Reduction schemes for one-loop tensor integrals", Nucl. Phys. B 73462 (2006) arXiv:hep-ph/0509141. S. Dittmaier, "Separation of soft and collinear singularities from one- loop N-point integrals", Nucl. Phys. B 675447 (2003) arXiv:hep-ph/0308246. Giele, W. T. and Glover, E. W. N., "A calculational formalism for one-loop integrals", JHEP 04 (2004) 029 arXiv:hepph/0402152]. Ellis, R. K. and Giele, W. T. and Zanderighi, G., "Virtual QCD corrections to Higgs boson plus four parton processes", Phys. Rev. D 72054018 (2005) arXiv:hep-ph/0506196]. - "Semi-numerical evaluation of one-loop corrections", arXiv:hep-ph/0508308. Binoth, T. and Guillet, J. P. and Heinrich, G., "Reduction formalism for dimensionally regulated one-loop N-point integrals", Nucl. Phys. B572 361 (2000) arXiv:hep-ph/9911342]. Binoth, T. and Guillet, J. Ph. and Heinrich, G. and Pilon, E. and Schubert, C., "An algebraic / numerical formalism for one-loop multi-leg amplitudes", JHEP 10015 (2005) [arXiv:hep-ph/0504267]. Boudjema, F. and others, "Multi-leg calculations with the GRACE/1-LOOP system: Toward radiative corrections to e+ e- -i. mu- anti-nu u anti-d", Nucl. Phys. Proc. Suppl. 135323 (2004) arXiv:hep-ph/0407079. Kurihara, Y., "Dimensionally regularized one-loop tensorintegrals with massless internal particles", arXiv:hep-ph/0504251'. del Aguila, F. and Pittau, R., "Recursive numerical calculus of one-loop tensor integrals", JHEP 07017 (2004) arXiv:hep-ph/0404120]. van Hameren, A. and Vollinga, J. and Weinzierl, S., "Automated computation of one-loop integrals in massless theories", Eur. Phys. J. C 41361 (2005) arXiv:hep-ph/0502165. Ferroglia, A., Passera, M., Passarino, G., and Uccirati, Sandro, "All-purpose numerical evaluation of one-loop multi-leg Feynman 
diagrams", Nucl. Phys. B 650162 (2003) [arXiv:hep-ph/0209219]. Binoth, T. and Heinrich, G. and Kauer, N., "A numerical evaluation of the scalar hexagon integral in the physical region", Nucl. Phys. B 654277 (2003) arXiv:hep-ph/0210023. Nagy, Z. and Soper, D. E., "General subtraction method for numerical calculation of oneloop QCD matrix elements", JHEP 09055 (2003) arXiv:hep-ph/0308127. - Acta Phys. Polon. B 352557 (2004). Kurihara, Y. and Kaneko, T., "Numerical contour integration for loop integrals", arXiv:hep-ph/0503003. Anastasiou, C. and Daleo, A., "Numerical evaluation of loop integrals", arXiv:hep-ph/0511176".

[6] F. A. Berends, R. Kleiss, P. De Causmaecker, R. Gastmans and T. T. Wu, "Single Bremsstrahlung Processes In Gauge Theories," Phys. Lett. B103 (1981) 124; P. De Causmaeker, R. Gastmans, W. Troost and T. T. Wu, "Multiple Bremsstrahlung In Gauge Theories At High-Energies. 1. General Formalism For Quantum Electrodynamics," Nucl. Phys. B206 (1982) 53; R. Kleiss and W. J. Stirling, "Spinor Techniques For Calculating P Anti-P $\rightarrow$ W+- / Z0 + Jets," Nucl. Phys. B262 (1985) 235; R. Gastmans and T. T. Wu, The Ubiquitous Photon: Helicity Method For QED And QCD Clarendon Press, 1990.

[7] Z. Xu, D.-H. Zhang and L. Chang, "Helicity Amplitudes For Multiple Bremsstrahlung In Massless Nonabelian Theories," Nucl. Phys. B291 (1987) 392.

[8] J. F. Gunion and Z. Kunszt, "Improved Analytic Techniques For Tree Graph Calculations And The G G Q Anti-Q Lepton Anti-Lepton Subprocess," Phys. Lett. 161B (1985) 333.

[9] L. J. Dixon, "Calculating Scattering Amplitudes Efficiently," hep-ph/9601359.

[10] Z. Bern, L. J. Dixon, D. C. Dunbar and D. A. Kosower, "One loop n point gauge theory amplitudes, unitarity and collinear limits," Nucl. Phys. B 425, 217 (1994) arXiv:hep-ph/9403226.

[11] Z. Bern, L. J. Dixon, D. C. Dunbar and D. A. Kosower, "Fusing gauge theory tree amplitudes into loop amplitudes," Nucl. Phys. B 435, 59 (1995) arXiv:hep-ph/9409265.

[12] R. Britto, E. Buchbinder, F. Cachazo and B. Feng, "One-loop amplitudes of gluons in SQCD," Phys. Rev. D 72, 065012 (2005) arXiv:hep-ph/0503132.

[13] Z. Bern, L. J. Dixon and D. A. Kosower, "Bootstrapping multi-parton loop amplitudes in QCD," arXiv:hep-ph/0507005.

[14] Z. Bern, L. J. Dixon and D. A. Kosower, "The last of the finite loop amplitudes in QCD," Phys. Rev. D 72, 125003 (2005) arXiv:hep-ph/0505055.

[15] D. Forde and D. A. Kosower, "All-multiplicity one-loop corrections to MHV amplitudes in QCD," arXiv:hep-ph/0509358.

[16] Z. Bern, L. J. Dixon and D. A. Kosower, "Progress in one-loop QCD computations," Ann. Rev. Nucl. Part. Sci. 46, 109 (1996) arXiv:hep-ph/9602280.

[17] E. Witten, "Perturbative gauge theory as a string theory in twistor space," Commun. Math. Phys. 252, 189 (2004) [arXiv:hep-th/0312171]. 
[18] F. Cachazo, P. Svrček and E. Witten, "MHV vertices and tree amplitudes in gauge theory," JHEP 0409, 006 (2004) arXiv:hep-th/0403047.

[19] F. Cachazo, P. Svrček and E. Witten, "Twistor space structure of one-loop amplitudes in gauge theory," JHEP 0410, 074 (2004) arXiv:hep-th/0406177.

[20] F. Cachazo, P. Svrček and E. Witten, "Gauge theory amplitudes in twistor space and holomorphic anomaly," JHEP 0410, 077 (2004) arXiv:hep-th/0409245.

[21] F. Cachazo, "Holomorphic Anomaly Of Unitarity Cuts And One-Loop Gauge Theory Amplitudes," hep-th/0410077.

[22] F. Cachazo and P. Svrcek, "Lectures on twistor strings and perturbative Yang-Mills theory," PoS RTN2005, 004 (2005) arXiv:hep-th/0504194].

[23] L. J. Dixon, "Twistor string theory and QCD," arXiv:hep-ph/0512111.

[24] Z. Bern, L. J. Dixon and D. A. Kosower, "One-loop amplitudes for e+ e- to four partons," Nucl. Phys. B 513, 3 (1998) arXiv:hep-ph/9708239.

[25] Z. Bern, L. J. Dixon and D. A. Kosower, "Two-loop g $\rightarrow$ g g splitting amplitudes in QCD," JHEP 0408, 012 (2004) hep-ph/0404293.

[26] Z. Bern, V. Del Duca, L. J. Dixon and D. A. Kosower, "All non-maximally-helicityviolating one-loop seven-gluon amplitudes in $\mathrm{N}=4$ super-Yang-Mills theory," Phys. Rev. D 71, 045006 (2005) arXiv:hep-th/0410224.

[27] R. Britto, F. Cachazo and B. Feng, "Generalized unitarity and one-loop amplitudes in N = 4 super-Yang-Mills," Nucl. Phys. B 725, 275 (2005) arXiv:hep-th/0412103.

[28] Z. Bern, L. J. Dixon and D. A. Kosower, "All next-to-maximally helicity-violating oneloop gluon amplitudes in $\mathrm{N}=4$ super-Yang-Mills theory," Phys. Rev. D 72, 045014 (2005) arXiv:hep-th/0412210.

[29] Z. Bern, J.S. Rozowsky and B. Yan, Phys, "Two-loop four-gluon amplitudes in N = 4 super-Yang-Mills," Phys. Lett. B 401, 273 (1997) hep-ph/9702424; Z. Bern, L. J. Dixon, D. C. Dunbar, M. Perelstein and J. S. Rozowsky, "On the relationship between Yang-Mills theory and gravity and its implication for ultraviolet divergences," Nucl. Phys. B 530, 401 (1998) hep-th/9802162; C. Anastasiou, Z. Bern, L. J. Dixon and D. A. Kosower, "Planar amplitudes in maximally supersymmetric Yang-Mills theory," Phys. Rev. Lett. 91, 251602 (2003) hep-th/0309040; Z. Bern, L. J. Dixon and V. A. Smirnov, "Iteration of planar amplitudes in maximally supersymmetric YangMills theory at three loops and beyond," Phys. Rev. D 72, 085001 (2005) arXiv:hepth/0505205.

[30] E. I. Buchbinder and F. Cachazo, "Two-loop amplitudes of gluons and octa-cuts in N = 4 super Yang-Mills," JHEP 0511, 036 (2005) arXiv:hep-th/0506126.

[31] Z. Bern and A. G. Morgan, "Massive Loop Amplitudes from Unitarity," Nucl. Phys. B 467, 479 (1996) arXiv:hep-ph/9511336.

[32] Z. Bern, L. J. Dixon and D. A. Kosower, "A two-loop four-gluon helicity amplitude in QCD," JHEP 0001, 027 (2000) arXiv:hep-ph/0001001]. Z. Bern, A. De Freitas 
and L. J. Dixon, "Two-loop amplitudes for gluon fusion into two photons," JHEP 0109, 037 (2001) [hep-ph/0109078]; "Two-loop helicity amplitudes for gluon gluon scattering in QCD and supersymmetric Yang-Mills theory," JHEP 0203, 018 (2002) hep-ph/0201161].

[33] S. Parke and T. Taylor, "An Amplitude For N Gluon Scattering," Phys. Rev. Lett. 56 (1986) 2459; F. A. Berends and W. T. Giele, "Recursive Calculations For Processes With $N$ Gluons," Nucl. Phys. B306 (1988) 759.

[34] M. Mangano and S. J. Parke, "Multiparton Amplitudes In Gauge Theories," Phys. Rep. 200 (1991) 301.

[35] R. Britto, F. Cachazo and B. Feng, "New recursion relations for tree amplitudes of gluons," Nucl. Phys. B 715, 499 (2005) [arXiv:hep-th/0412308].

[36] R. Britto, F. Cachazo, B. Feng and E. Witten, "Direct proof of tree-level recursion relation in Yang-Mills theory," Phys. Rev. Lett. 94, 181602 (2005) arXiv:hep-th/0501052.

[37] A. Brandhuber, B. Spence and G. Travaglini, "One-Loop Gauge Theory Amplitudes In $\mathrm{N}=4$ Super Yang-Mills From MHV Vertices," Nucl. Phys. B 706, 150 (2005) arXiv:hep-th/0407214; C. Quigley and M. Rozali, "One-loop MHV amplitudes in supersymmetric gauge theories," JHEP 0501, 053 (2005) arXiv:hep-th/0410278; J. Bedford, A. Brandhuber, B. Spence and G. Travaglini, "A twistor approach to oneloop amplitudes in $\mathrm{N}=1$ supersymmetric Yang-Mills theory," Nucl. Phys. B 706, 100 (2005) arXiv:hep-th/0410280; Y. t. Huang, "N = 4 SYM NMHV loop amplitude in superspace," Phys. Lett. B 631, 177 (2005) [arXiv:hep-th/0507117. A. Brandhuber, B. Spence and G. Travaglini, "From trees to loops and back," JHEP 0601, 142 (2006) arXiv:hep-th/0510253.

[38] R. Britto, F. Cachazo and B. Feng, "Computing one-loop amplitudes from the holomorphic anomaly of unitarity cuts," Phys. Rev. D 71, 025012 (2005) arXiv:hepth/0410179].

[39] S. J. Bidder, N. E. J. Bjerrum-Bohr, L. J. Dixon and D. C. Dunbar, "N = 1 supersymmetric one-loop amplitudes and the holomorphic anomaly of unitarity cuts," Phys. Lett. B 606, 189 (2005) arXiv:hep-th/0410296].

[40] I. Bena, Z. Bern, D. A. Kosower and R. Roiban, "Loops in twistor space," Phys. Rev. D 71, 106010 (2005) arXiv:hep-th/0410054.

[41] S. J. Bidder, N. E. J. Bjerrum-Bohr, D. C. Dunbar and W. B. Perkins, "Twistor space structure of the box coefficients of $\mathrm{N}=1$ one-loop amplitudes," Phys. Lett. B 608, 151 (2005) arXiv:hep-th/0412023.

[42] S. J. Bidder, N. E. J. Bjerrum-Bohr, D. C. Dunbar and W. B. Perkins, "One-loop gluon scattering amplitudes in theories with $\mathrm{N} ; 4$ supersymmetries," Phys. Lett. B 612, 75 (2005) arXiv:hep-th/0502028.

[43] Z. Bern, L. Dixon and D. A. Kosower, "New QCD Results From String Theory," in Strings '93, ed. M. B. Halpern et. al. (World-Scientific, 1995), hep-th/9311026. 
[44] Z. Bern, G. Chalmers, L. J. Dixon and D. A. Kosower, "One Loop N Gluon Amplitudes with Maximal Helicity Violation via Collinear Limits," Phys. Rev. Lett. 72 (1994) 2134.

[45] G. Mahlon, "Multi - gluon helicity amplitudes involving a quark loop," Phys. Rev. D 49, 4438 (1994) arXiv:hep-ph/9312276.

[46] J. Bedford, A. Brandhuber, B. J. Spence and G. Travaglini, "Non-supersymmetric loop amplitudes and MHV vertices," Nucl. Phys. B 712, 59 (2005) arXiv:hep-th/0412108.

[47] Z. Bern, N. E. J. Bjerrum-Bohr, D. C. Dunbar and H. Ita, "Recursive calculation of one-loop QCD integral coefficients," JHEP 0511, 027 (2005) arXiv:hep-ph/0507019.

[48] Z. Bern, L. J. Dixon, D. C. Dunbar and D. A. Kosower, "One-loop self-dual and N = 4 super-Yang-Mills," Phys. Lett. B 394, 105 (1997) [hep-th/9611127]; Z. Bern, L. J. Dixon, M. Perelstein and J. S. Rozowsky, "Multi-leg one-loop gravity amplitudes from gauge theory," Nucl. Phys. B 546, 423 (1999) hhep-th/9811140.

[49] A. Brandhuber, S. McNamara, B. J. Spence and G. Travaglini, "Loop amplitudes in pure Yang-Mills from generalised unitarity," JHEP 0510, 011 (2005) arXiv:hepth/0506068].

[50] S. D. Badger, E. W. N. Glover, V. V. Khoze and P. Svrcek, "Recursion relations for gauge theory amplitudes with massive particles," JHEP 0507, 025 (2005) arXiv:hepth/0504159].

[51] S. D. Badger, E. W. N. Glover and V. V. Khoze, "Recursion relations for gauge theory amplitudes with massive vector bosons and fermions," JHEP 0601, 066 (2006) arXiv:hep-th/0507161.

[52] D. Forde and D. A. Kosower, "All-multiplicity amplitudes with massive scalars," arXiv:hep-th/0507292.

[53] G. Rodrigo, "Multigluonic scattering amplitudes of heavy quarks," JHEP 0509, 079 (2005) arXiv:hep-ph/0508138.

[54] C. Quigley and M. Rozali, "Recursion relations, helicity amplitudes and dimensional regularization," arXiv:hep-ph/0510148.

[55] P. Ferrario, G. Rodrigo and P. Talavera, "Compact multigluonic scattering amplitudes with heavy scalars and fermions," arXiv:hep-th/0602043.

[56] Z. Bern, L. J. Dixon and D. A. Kosower, "On-shell recurrence relations for one-loop QCD amplitudes," Phys. Rev. D 71, 105013 (2005) hep-th/0501240].

[57] Z. Bern, L. J. Dixon and D. A. Kosower, "Dimensionally Regulated Pentagon Integrals," Nucl. Phys. B 412, 751 (1994), hep-ph/9306240.

[58] Z. Kunszt and D. Soper, "Calculation of jet cross-sections in hadron collisions at order alpha-s**3,"Phys. Rev. D46 (1992) 192; Z. Kunszt, A. Signer and Z. Trócsányi, "Singular terms of helicity amplitudes at one loop in QCD and the soft limit of the cross-sections of multiparton processes," Nucl. Phys. B420 (1994) 550. 
[59] W. T. Giele and E. W. N. Glover, "Higher order corrections to jet cross-sections in e+ e- annihilation," Phys. Rev. D46 (1992) 1980; W. T. Giele, E. W. N. Glover and D. A. Kosower, "Higher order corrections to jet cross-sections in hadron colliders," Nucl. Phys. B403 (1993) 633.

[60] Z. Bern and G. Chalmers, "Factorization in one loop gauge theory," Nucl. Phys. B 447, 465 (1995) arXiv:hep-ph/9503236.

[61] L.D. Landau, Nucl. Phys. 13, 181 (1959); S. Mandelstam, Phys. Rev. 112, 1344 (1958), 115, 1741 (1959); R.E. Cutskosky, J. Math. Phys. 1, 429 (1960).

[62] R. J. Eden, P. V. Landshoff, D. I. Olive and J. C. Polkinghorne, The Analytic S-Matrix, Cambridge University Press, 1966.

[63] M. T. Grisaru, H. N. Pendleton and P. van Nieuwenhuizen, "Supergravity And The S Matrix," Phys. Rev. D15 (1977) 996; M. T. Grisaru and H. N. Pendleton, "Some Properties Of Scattering Amplitudes In Supersymmetric Theories," Nucl. Phys. B124 (1977) 81.

[64] M. x. Luo and C. k. Wen, "Compact formulas for all tree amplitudes of six partons," Phys. Rev. D 71, 091501 (2005) arXiv:hep-th/0502009.

[65] M. x. Luo and C. k. Wen, "Recursion relations for tree amplitudes in super gauge theories," JHEP 0503, 004 (2005) arXiv:hep-th/0501121. 